School of Finance

University of St.Gallen

«DIVERSIFYING ESTIMATION ERRORS:

AN EFFICIENT AVERAGING RULE FOR PORTFOLIO

OPTIMIZATION»

ROLAND FÜSS

CHRISTIAN KOEPPEL

FELIX Miebs

WORKING PAPERS ON FINANCE NO. 2021/05

SWISS INSTITUTE OF BANKING AND FINANCE (S/BF - HSG)

FEBRUARY $8^{\mathrm{TH}}, 2021$ 


\title{
Diversifying estimation errors: An efficient averaging rule for portfolio optimization
}

\author{
Roland Füss* $\quad$ Christian Koeppel ${ }^{\dagger} \quad$ Felix Miebs ${ }^{\ddagger}$
}

February 8, 2021

\begin{abstract}
We propose an averaging rule that combines established minimum-variance strategies to minimize the expected out-of-sample variance. Our rule overcomes the problem of selecting the "best" strategy ex-ante and diversifies remaining estimation errors of the single strategies included in the averaging. Extensive simulations show that the contributions of estimation errors to the out-of-sample variances are uncorrelated between the considered strategies. This implies that averaging over multiple strategies offers sizable diversification benefits. Our rule leverages these benefits and compares favorably to eleven strategies in terms of out-of-sample variance on both simulated and empirical data sets. The Sharpe ratio is across all data sets at least $25 \%$ higher than for the $1 / \mathrm{N}$ portfolio.
\end{abstract}

JEL Classification: G11

Keywords: Averaging; diversification; estimation error; portfolio optimization; shrinkage.

\footnotetext{
*Swiss Institute of Banking and Finance (s/bf), University of St. Gallen, Unterer Graben 21, 9000 St.Gallen, Switzerland, Visiting Professor and Fellow at the Center for Real Estate and Environmental Economics at NTNU Business School, Trondheim, Norway; Phone: +41 (0)71 224-7055, Fax: +41 (0)71 224-7088; Email: roland.fuess@unisg.ch

${ }^{\dagger}$ University of St.Gallen, Unterer Graben 21, 9000 St. Gallen, Switzerland; Phone: +41 (0)76 3830 757, Email: christian.koeppel@live.com

${ }^{\ddagger}$ University of Applied Sciences Cologne, Claudiusstr. 1, 50678 Cologne, Germany; Phone: +49 (0)221 8275 3792; Email: felix.miebs@th-koeln.de.
} 


\section{Introduction}

The literature on portfolio selection offers a broad range of approaches to alleviate the impact of estimation errors on out-of-sample portfolio performance. Nevertheless, DeMiguel et al. (2009b) find that no single portfolio strategy consistently outperforms the $1 / \mathrm{N}$ rule. Stimulated by this observation, several authors suggested improved estimates of the input parameters on the covariance (see, e.g., Fan et al., 2013; Ledoit and Wolf, 2017), the inverse covariance (see, e.g., Kourtis et al., 2012, DeMiguel et al., 2013, Shi et al., 2020) and the portfolio weight level (see, e.g., Kan and Zhou, 2007, Tu and Zhou, 2011; DeMiguel et al., 2013). Further contributions propose extensions of the portfolio optimization problem via norm constraints in order to regularize it (see, e.g., Brodie et al., 2009, DeMiguel et al., 2009b; Xing et al., 2014, Li, 2015, Yen, 2016). The plethora of evolving strategies challenges practitioners and academics alike to choose one of the proposed strategies. In addition to the challenge of identifying the "best" strategy ex-ante, the limitation to a single strategy may forego potential diversification benefits. If estimation errors are not perfectly correlated across individual strategies, a combination of different approaches offers the potential to further diversify estimation errors. The resulting portfolio weights are less prone to estimation errors and achieve, on average, an improved out-of-sample performance.

In this paper, we propose an averaging rule that overcomes the strategy selection problem and exploits the potential to diversify estimation errors between individual strategies. Our approach takes the principle of diversification from the portfolio to the estimation level. Following the consensus in the literature, we apply our averaging rule to minimum-variance portfolios because expected returns are notoriously difficult to estimate (see, e.g., Merton, 1980).

Our rule aims to minimize the expected out-of-sample portfolio variance. For this purpose and to reduce the impact of estimation errors, we combine established approaches on three different levels: i) the covariance level, where averaging is applied before one computes the inverse of the covariance matrix, ii) the inverse covariance level, where averaging is applied on the individual inverses of the various covariance matrices, and iii) the portfolio weight level, where averaging is applied directly on the portfolio weights. Our averaging rule combines the unbiased sample estimator with a multitude of structured estimators 1 Thus, our approach extends the idea of shrinkage from a single to a multi-target specification.

\footnotetext{
${ }^{1}$ Throughout the paper, we average over three different levels (covariance, inverse, and weight level), use the Sample and the structured (target) estimators (jointly denoted as strategies) for averaging on the aforementioned levels, and benchmark our rule against existing shrinkage approaches (denoted as benchmarks).
} 
In extensive simulations, we find that estimation errors between a variety of established minimum-variance strategies are uncorrelated which is undocumented and unexploited so far. This correlation structure offers sizable and persistent diversification benefits. Our averaging rule utilizes this structure to diversify estimation errors, leading to a decrease in the out-of-sample variance as the number of strategies increases. The average out-of-sample variance of our rule over all simulations is lower than the variance of any single strategy. Our averaging rule also achieves higher Sharpe ratios for smaller estimation windows. In addition, we find that our rule compares favorably to the selected benchmarks, not only in terms of lower out-of-sample variance, but also higher out-of-sample Sharpe ratio. This includes the most recent non-linear shrinkage approach of Ledoit and Wolf (2017).

Empirical results on five data sets confirm the findings from the simulation study that our rule achieves a lower out-of-sample variance than any single strategy. The variance reduction in comparison to the non-linear shrinkage strategy of Ledoit and Wolf $(2017)$ is statistically significant on three data sets. Our rule achieves the second highest Sharpe ratio on all data sets in comparison to the single strategies. Relative to the $1 / \mathrm{N}$ strategy, the out-of-sample Sharpe ratio of our rule is on all data sets at least $25 \%$ higher, being statistically significant in four out of five sets. The turnover and short interest of our averaging rule is modest. Averaging consistently outperforms the single strategies and benchmarks in terms of risk reduction and performance improvement, as well as reduction in turnover and short interest. These properties make our averaging rule both statistically and economically appealing.

Our paper relates to prior contributions proposing combinations of portfolio weights or input parameters to reduce estimation errors. Tu and Zhou (2011) show that combining portfolio weights of established mean-variance strategies with the $1 / \mathrm{N}$ rule improves the out-of-sample performance of the respective strategies. Kourtis et al. (2012) find that a linear combination of the sample and two structured estimators on the inverse covariance level improves the out-of-sample performance of minimum-variance portfolios. The results of the aforementioned articles suggest that estimation errors may further be reduced, if just one additional resource for reducing errors is included. We follow this path, exploring whether estimation errors can be diversified more comprehensively if multiple resources for the diversification are taken into account.

We contribute to the existing literature in two ways. First, in a battery of simulations, we find that estimation errors between a variety of established minimum-variance strategies are 
uncorrelated which is undocumented and unexploited so far. Our findings show that the resulting diversification benefits are sizable and persistent for different estimation windows. Second, we provide a framework for combining multiple strategies to alleviate the impact of estimation errors. Our averaging rule builds on the principle of diversification, is easy to calibrate, and extends to an arbitrary number of strategies, not limited to the ones selected for this paper. On simulated and empirical data our rule compares favorably to selected benchmarks in terms of out-of-sample variance and Sharpe ratio. Thus, averaging over single strategies provides a promising avenue to reduce the impact of estimation errors on out-of-sample portfolio performance.

The remainder of the paper is organized as follows. Section 2 reviews the considered minimum-variance strategies and introduces our averaging rule. Section 3 describes the simulation procedure and explores the behavior of the averaging rule using simulated data. Section 4 evaluates the out-of-sample performance of our averaging rule on five empirical data sets. Section 5 concludes.

\section{Portfolio optimization and estimation errors}

\subsection{Minimum-variance portfolio optimization}

Throughout the paper we focus on the estimation of the minimum-variance portfolio resulting from the following optimization problem:

$$
\begin{array}{ll}
\min _{w} & w^{\prime} \Sigma w \\
\text { s.t. } & w^{\prime} \mathbf{1}_{N}=1,
\end{array}
$$

where $\Sigma$ is the true but unknown covariance matrix, $\mathbf{1}_{N}$ is a $N$-dimensional vector of ones, and $N$ is the number of investable risky assets, respectively. The allocation to the $N$ assets within the minimum-variance portfolio results from solving the problem in Equation (1), with the optimal portfolio weights given by:

$$
w=\Sigma^{-1} \mathbf{1}_{N}\left(\mathbf{1}_{N}^{\prime} \Sigma^{-1} \mathbf{1}_{N}\right)^{-1}
$$

Because $w$ depends on the true but unknown covariance matrix, $\Sigma$ has to be replaced by a

suitable estimator, $\hat{\Sigma}$. A natural estimator for the covariance matrix is the sample covariance 
matrix. Nevertheless, the estimation error associated with the sample covariance matrix can be substantial (see, e.g., Ledoit and Wolf, 2003, DeMiguel et al., 2009b). Any estimation errors in $\hat{\Sigma}$ or its inverse, translate into sub-optimal allocations with an adverse effect on the portfolio performance.

\subsection{Alleviating estimation errors: Existing approaches}

The literature proposes different approaches to mitigate the impact of estimation errors on minimum-variance portfolio weights and out-of-sample performance. The first branch of literature suggests structured estimators of the covariance matrix. Examples include the constant correlation model (see, e.g., Elton and Gruber, 1973) and (approximate) factor models (see, e.g., Chan et al., 1999, Fan et al., 2013). The second branch proposes shrinkage estimators of the covariance matrix (see, e.g., Ledoit and Wolf, 2003, 2004a b, 2017), its inverse (see, e.g., Kourtis et al., 2012; DeMiguel et al., 2013, Shi et al., 2020), and portfolio weights (see, e.g., Kan and Zhou, 2007, Tu and Zhou, 2011, DeMiguel et al., 2013). The third branch regularizes portfolio weights through the imposition of weight constraints. Frost and Savarino (1986) as well as Jagannathan and Ma (2003), study the effects of short-sale constraint. Brodie et al. (2009), DeMiguel et al. (2009b), Xing et al. (2014), Li (2015), and Yen (2016) suggest the imposition of norm constraints on portfolio weights.2

Throughout the paper we report results for eleven minimum-variance strategies, covering at least one representative of each aforementioned branch from the existing literature. Each of these can be represented in terms of a covariance matrix estimator. The portfolio weights of each strategy are given by Equation (2), with $\hat{\Sigma}$ being one of the following estimators, clustered into two groups $3^{3}$ The first group comprises the sample as well as structured estimates of the covariance matrix and represents the building blocks for our averaging rule:

- Sample: The (unbiased) sample covariance matrix, $\hat{\Sigma}_{S}$.

- 1F: The covariance matrix implied by the single factor model of Sharpe $(1967), \hat{\Sigma}_{1 F}$.

- CC: The covariance matrix recovered from a constant correlation matrix with the average of all pairwise correlations off the main diagonal and the assets' individual standard

\footnotetext{
${ }^{2}$ We do not include norm-constrained strategies in our paper because the moment-shrinkage representation of norm constraints does not hold for arbitrary levels of the constraint. Thus, it is not possible to use the aforementioned approaches on the three considered averaging levels.

${ }^{3}$ We provide a comprehensive review of the strategies in section A.1 of the Internet Appendix.
} 
deviations, $\hat{\Sigma}_{C C}$.

- ID: A diagonal matrix with the average variance of all assets on the main diagonal, $\hat{\Sigma}_{I D}$. The resulting minimum-variance portfolio corresponds to the $1 / \mathrm{N}$ portfolio, which represents a benchmark in terms of risk-adjusted performance (DeMiguel et al., 2009b).

- SC: The covariance matrix corresponding to the short-sale constrained minimum-variance portfolio from Jagannathan and Ma (2003), implying non-negative portfolio weights, $\hat{\Sigma}_{S C}$.

- POET: The covariance matrix based on the approximate factor model by Fan et al. (2013) with risk factors defined by the principal components of the sample covariance matrix, $\hat{\Sigma}_{P O E T}$.

The second group of estimators covers combinations of the sample and another covariance estimator serving as benchmarks for our averaging rule:

- LW1F: A linear combination of $\hat{\Sigma}_{S}$ and $\hat{\Sigma}_{1 F}$. The combination intensity is determined by the linear shrinkage estimator of Ledoit and Wolf (2003).

- LWID: A linear combination of $\hat{\Sigma}_{S}$ and $\hat{\Sigma}_{I D}$. The combination intensity is determined by the linear shrinkage estimator of Ledoit and Wolf (2004a).

- LWCC: A linear combination of $\hat{\Sigma}_{S}$ and $\hat{\Sigma}_{C C}$. The combination intensity is determined by the linear shrinkage estimator of Ledoit and Wolf (2004b).

- LWNLS: A non-linear shrinkage estimator, correcting for over-dispersed eigenvalues of the sample covariance matrix following Ledoit and Wolf (2017). This approach represents a benchmark in terms of out-of-sample variance.

- KDM: A linear combination of $\hat{\Sigma}_{S}, \hat{\Sigma}_{1 F}$ and $\hat{\Sigma}_{C C}$. The combination intensity is determined following Kourtis et al. (2012).

All approaches aim to determine the single best strategy to alleviate the impact of estimation errors. The wide variety of approaches leaves investors with the question of which suggested strategy to follow. Without any ex-ante knowledge about the "best" approach, investors face the problem of picking a single strategy. In contrast to choosing one single strategy, we advocate that investors should rather diversify over various strategies to mitigate estimation errors. 


\subsection{Estimation error diversification: An averaging approach}

Our approach of estimation error diversification generalizes the idea of shrinkage. We suggest blending the sample with a multitude of structured estimators. If estimation errors can be reduced by combining the sample with one structured estimator, averaging over multiple estimators should offer further diversification potential. In essence, our approach brings the principle of diversification from the portfolio to the estimation level.

We suggest an averaging rule that combines individual estimators with the objective of minimizing the expected out-of-sample variance of the corresponding minimum-variance portfolio 4 Because there is no consensus in the literature whether estimation errors can most efficiently be diversified on the covariance, inverse or portfolio weight level, we consider averaging over estimators on the three aforementioned levels. We assign the averaging weights to the considered estimators on the respective level such that the expected out-of-sample variance of the corresponding minimum-variance portfolio is minimized:

$$
\begin{array}{ll}
\min _{\lambda_{\text {Level }}} & E\left(\hat{w}_{\text {Level }}\left(\lambda_{\text {Level }}\right)^{\prime} \Sigma \hat{w}_{\text {Level }}\left(\lambda_{\text {Level }}\right)\right) \\
\text { s.t. } & \lambda_{\text {Level }}^{\prime} \mathbf{1}_{M}=1 \\
& \lambda_{\text {Level }} \geq 0
\end{array}
$$

where $\lambda_{\text {Level }}$ are the averaging weights on the respective averaging level, $\hat{w}_{\text {Level }}$ are the corresponding portfolio weight estimates, $\mathbf{1}_{M}$ is a $M$-dimensional vector of ones, and $M$ is the number of estimators. Equations (3) - (5) correspond to a minimum-variance portfolio optimization problem with non-negativity restrictions on the averaging weights. We impose the non-negativity constraint because Jagannathan and Ma (2003) show that it has a shrinkage-like effect and improves the portfolio performance. The literature on linear regression model averaging also finds that non-negativity constraints enhance the quality of the combination (see, e.g., Timmermann, 2006, Hansen, 2008). 5

If averaging is conducted on the covariance or inverse level, we combine the $M$ estimators of the covariance matrix or its inverse in a first step and compute the corresponding minimum-

\footnotetext{
${ }^{4}$ We do not claim that other, potentially more sophisticated averaging rules may deliver improved results. We rather advocate in a comparatively simple setting that averaging over individual established approaches provides a promising avenue to alleviate the impact of estimation errors on portfolio performance.

5 Britten-Jones (1999) shows that the mean-variance portfolio optimization problem can be formulated as a linear regression problem.
} 
variance portfolio weights in a second step. Following the objective in Equation (3), we combine the individual estimators such that the expected out-of-sample variance of the corresponding minimum-variance portfolio is minimized. We approximate the expected out-of-sample variance based on jackknifed returns, considering a one-month holding period throughout the paper (see, e.g., DeMiguel et al., 2009a; Xing et al., 2014, Li, 2015, Yen, 2016). We drop the excess returns falling into the $i$-th month from our in-sample period and compute the covariance or its inverse, respectively, using the remaining excess returns within the in-sample period. We denote the $m$-th estimator of the covariance or its inverse, by $\hat{\Sigma}_{-i, m}$ and $\hat{\Sigma}_{-i, m}^{-1}$, respectively. The minimum-variance portfolio weights corresponding to the combination of the $M$ estimators of the covariance matrix, $\hat{w}_{-i, A V-C o v}$, and its inverse, $\hat{w}_{-i, A V-I n v}$, are given by:

$$
\begin{aligned}
& \hat{w}_{-i, A V-C o v}=\left(\sum_{m=1}^{M} \hat{\lambda}_{m, C o v} \cdot \hat{\Sigma}_{-i, m}\right)^{-1} \mathbf{1}_{N}\left(\mathbf{1}_{N}^{\prime}\left(\sum_{m=1}^{M} \hat{\lambda}_{m, C o v} \cdot \hat{\Sigma}_{-i, m}\right)^{-1} \mathbf{1}_{N}\right)^{-1}, \\
& \hat{w}_{-i, A V-I n v}=\left(\sum_{m=1}^{M} \hat{\lambda}_{m, I n v} \cdot \hat{\Sigma}_{-i, m}^{-1}\right) \mathbf{1}_{N}\left(\mathbf{1}_{N}^{\prime} \sum_{m=1}^{M} \hat{\lambda}_{m, I n v} \cdot \hat{\Sigma}_{-i, m}^{-1} \mathbf{1}_{N}\right)^{-1},
\end{aligned}
$$

where $\hat{\lambda}_{C o v}$ and $\hat{\lambda}_{\text {Inv }}$ are our estimates of the averaging weights. We then compute the jackknife excess returns for the $i$-th month, $r_{i, \text { Cov }}^{J K}$ and $r_{i, I n v}^{J K}$, representing the excess returns that would have been achieved from holding $\hat{w}_{-i, \text { Cov }}$ and $\hat{w}_{-i, I n v}$, respectively, in the hold-out month $i$. Repeating these steps for all months within our in-sample period yields the time series of jackknifed portfolio excess returns. We use the sample variance of this time series as an estimate of the objective in Equation (3). The portfolio weights resulting from this averaging procedure are given by:

$$
\begin{aligned}
\hat{w}_{A V-C o v} & =\left(\sum_{m=1}^{M} \hat{\lambda}_{m, C o v} \cdot \hat{\Sigma}_{m}\right)^{-1} \mathbf{1}_{N}\left(\mathbf{1}_{N}^{\prime}\left(\sum_{m=1}^{M} \hat{\lambda}_{m, C o v} \cdot \hat{\Sigma}_{m}\right)^{-1} \mathbf{1}_{N}\right)^{-1}, \\
\hat{w}_{A V-I n v} & =\left(\sum_{m=1}^{M} \hat{\lambda}_{m, I n v} \cdot \hat{\Sigma}_{m}^{-1}\right)^{1} \mathbf{1}_{N}\left(\mathbf{1}_{N}^{\prime} \sum_{m=1}^{M} \hat{\lambda}_{m, \text { Inv }} \cdot \hat{\Sigma}_{m}^{-1} \mathbf{1}_{N}\right)^{-1},
\end{aligned}
$$

where $\hat{\Sigma}_{m}$ and $\hat{\Sigma}_{m}^{-1}$ denote the $m$-th estimator of the covariance or its inverse, based on all in-sample observations, respectively.

If averaging is conducted on the portfolio weight level, we combine the $M$ estimators of the minimum-variance portfolio weights such that the expected out-of-sample variance in Equation (3) is minimized. We follow the outlined jackknife procedure and drop the excess returns falling 
into the $i$-th month. Thereafter, we compute the minimum-variance portfolio weights $\hat{w}_{-i, m}$ for each of the $M$ estimators. The minimum-variance portfolio weights of the combination, $\hat{w}_{-i, A V-W g t}$, in the hold-out month $i$ are given by:

$$
\hat{w}_{-i, A V-W g t}=\sum_{m=1}^{M} \hat{\lambda}_{m, W g t} \cdot \hat{w}_{-i, m},
$$

where $\hat{\lambda}_{W g t}$ are the estimated averaging weights and $r_{i, A V-W g t}^{J K}$ is the jackknife return for the $i$-th month. Repeating the procedure for all months and computing the sample variance of the time series of jackknife excess returns again gives the estimate of the expected out-of-sample variance in Equation (3). The portfolio weights corresponding to averaging on the weight level are given by:

$$
\hat{w}_{A V-W g t}=\sum_{m=1}^{M} \hat{\lambda}_{m, W g t} \cdot \hat{w}_{m} .
$$

Table 1 provides an overview of our proposed averaging rule on the three levels in Panel A and the single strategies in Panel B. Panel C lists the five selected benchmarks of which LWNLS is the latest and most sophisticated, and thus our target to beat in terms of out-of-sample variance.

Throughout the paper we consider averaging over the sample and the $1 \mathrm{~F}, \mathrm{CC}, \mathrm{ID}, \mathrm{SC}$, and POET estimates on the three aforementioned levels. The selected set of estimators is, as any other set, an ad-hoc selection for which the motivation is twofold. First, we seek to build our averaging rule on an established set of simple estimators. Second, we want to include a sufficiently large number of estimators to explore the trade-off between a higher potential to diversify estimation errors and the newly arising estimation problem. Including more estimators in the averaging rule offers a greater potential to diversify estimation errors. Yet, including more strategies requires the estimation of more averaging weights, giving rise to a new source of estimation errors. We explore this trade-off in the following simulation study.

[TABLE 1 ABOUT HERE] 


\section{Simulation study}

\subsection{Simulation procedure}

The data generating process of our simulation study follows Tu and Zhou (2011). We consider $N=25$ assets with the simulated excess return of security $j$ at time $t, r_{j, t}$, coming from the Fama-French three-factor model with mispricing:

$$
r_{j, t}=\alpha_{j}+\beta_{j, M K T} r_{t, M K T}+\beta_{j, S M B} r_{t, S M B}+\beta_{j, H M L} r_{t, H M L}+u_{j, t},
$$

where $\beta_{j, M K T}, \beta_{j, S M B}$, and $\beta_{j, H M L}$ are the factor loadings, $\alpha_{j}$ is the mispricing factor, and $u_{j, t}$ is the residual at time $t$. The returns of the market $r_{t, M K T}$, size $r_{t, S M B}$, and value factor $r_{t, H M L}$ at $t$ follow a multivariate normal distribution. Table 2 shows the means and standard deviations of the factors as well as the correlation matrix between the factors.

\section{[TABLE 2 ABOUT HERE]}

The assets' factor loadings are randomly paired and evenly spread between 0.9 and 1.2 for $\beta_{M K T},-0.3$ and 1.4 for $\beta_{S M B},-0.5$ and 0.9 for $\beta_{H M L}$, and -2.0 and $2.0 \%$ for the annualized mispricing factor $\alpha$, in each simulation. The error term of each asset at $t, u_{j, t}$, comes from a multivariate normal distribution with $N \sim\left(0, \Sigma_{u}\right)$. Again, we follow Tu and Zhou (2011) and assume that the covariance matrix $\Sigma_{u}$ is diagonal. The residuals' annualized volatility is drawn from a uniform distribution with a lower bound of $10 \%$ and an upper bound of $30 \%$, such that the average idiosyncratic volatility in the cross-section equals $20 \%$.

We run 10,000 simulations. In each run $k$ we draw $\tau=\{60,120,240,480,960\}$ monthly observations from the outlined data generating process and save the population mean vector, $\mu_{k}$, and the covariance matrix, $\Sigma_{k}$. These parameters result from the random pairing of the assets' factor loadings, their mispricing, as well as their idiosyncratic volatility. We then estimate the portfolio weights of the $m$-th strategy, $\hat{w}_{k, m}$, based on the $\tau$ simulated excess returns and evaluate the estimated portfolio weights on the out-of-sample variance, $\hat{\sigma}_{k, m}$, and Sharpe ratio, $\hat{\Psi}_{k, m}$, of this simulation run:

$$
\begin{aligned}
\hat{\sigma}_{k, m}^{2} & =\hat{w}_{k, m}^{\prime} \Sigma_{k} \hat{w}_{k, m}, \\
\hat{\Psi}_{k, m} & =\frac{\hat{w}_{k, m}^{\prime} \mu_{k}}{\sqrt{\hat{w}_{k, m} \Sigma_{k} \hat{w}_{k, m}}} .
\end{aligned}
$$




\subsection{Simulation results}

In a first step, we investigate the estimation errors of the individual strategies. We quantify the contribution of the estimation errors to the out-of-sample variances of the $m$-th strategy in the $k$-th simulation run as the following loss:

$$
L_{k, m}=\left(\hat{w}_{k, m}-w_{k}\right)^{\prime} \Sigma_{k}\left(\hat{w}_{k, m}-w_{k}\right)
$$

where $w_{k}$ are the true minimum-variance portfolio weights, which we compute using the population covariance matrix of the respective run, $\Sigma_{k}$. Table 3 reports the means, standard deviations, and correlation coefficients between the losses of the single strategies over the 10,000 simulation runs. The means of all strategies decrease for increasing estimation windows in Panels A - E, except for the ID strategy. As expected, this decrease is most pronounced for the unbiased Sample strategy. The ID strategy corresponds to the $1 / \mathrm{N}$ portfolio, such that the means and standard deviations are constant over the estimation windows. The standard deviations of the other strategies decrease with larger sample sizes, being highest for the Sample for $\tau=60$ and smallest for $\tau=960$. Most importantly, the correlations are small in absolute terms, offering sizable diversification opportunities. The average pair-wise correlation reduces from an already low level of 0.173 to 0.046 going from $\tau=60$ to $\tau=960$. The minimum and maximum pair-wise correlation between the considered strategies range from -0.345 to 0.745 and -0.392 to 0.394 for $\tau=60$ and $\tau=960$, respectively. We observe the highest correlation between the two (approximate) factor model strategies POET and 1F, highlighting the need for heterogeneous strategies to lever the diversification potential. In contrast to existing linear shrinkage approaches that combine the Sample with one or two strategies (see, e.g., Ledoit and Wolf, 2003, 2004a b Kourtis et al., 2012), our results suggest that combining a multitude of strategies offers sizable diversification benefits. Yet, averaging over multiple strategies gives rise to a new estimation problem, resulting in the following trade-off: on the one hand, increasing the number of strategies lowers the portfolio variance; on the other hand, it increases the number of averaging weights that need to be estimated, creating a new source of estimation errors.

[TABLE 3 ABOUT HERE]

We evaluate this trade-off in Table 4 by applying our averaging rule to the sample covariance matrix and an increasing number of strategies from Panel B of Table 1 . Importantly, we consider 
all possible permutations that result from combining the sample covariance matrix with the respective subset of strategies ${ }^{6}$ For each permutation in each simulation run we compute the averaging weights according to our averaging rule and obtain the resulting portfolio weights. We then evaluate the estimated portfolio weights based on their out-of-sample variance according to Equation (10).

Table 4 shows the average out-of-sample variances over all simulations and permutations for the corresponding number of strategies. Panels A-E present the results for the different estimation windows $\tau=\{60,120,240,480,960\}$, when averaging is conducted on the portfolio weight (AV-Wgt), the inverse (AV-Inv), or the covariance (AV-Cov) level. Our results show that the average out-of-sample variance decreases as the number of strategies increases. $7^{7}$ This result holds irrespective of the estimation window and the averaging level, demonstrating that the diversification benefits outweigh the newly created estimation error problem.

\section{[TABLE 4 ABOUT HERE]}

Investigating the relevance of the single strategies within our averaging rule, we turn to Figure 1. This figure shows the average weights of the considered strategies over all simulations when averaging is conducted over $M=6$ strategies. Panel A displays the averaging weights for AV-Wgt, Panels B and C show the respective weights for AV-Inv and AV-Cov. We observe similar patterns for the three averaging levels. Our rule utilizes, on average, the multitude of strategies, corroborating their relevance for an efficient diversification of estimation errors. Turning to the weighting of the individual strategies, we observe that the weight of the Sample is low when the estimation window is small and vice versa. The average loss of the Sample is high (low) for smaller (higher) estimation windows, offering higher (lower) diversification gains. These higher (lower) gains are leveraged by assigning higher (lower) weights to the remaining strategies. This is in line with Table 3 where the average loss of the Sample decreases as the estimation window increases.

\section{[FIGURE 1]ABOUT HERE]}

\footnotetext{
${ }^{6}$ When averaging over the sample and another strategy, we have $M=2$, which gives rise to five permutations. The number of permutations for $M=3$ and $M=4$ amounts to ten, respectively, for $M=5$ to five, and for $M=6$ to one.

${ }^{7}$ We evaluate the distribution of the out-of-sample variances over all simulation runs and permutations for AV-Wgt, AV-Inv, and AV-Cov using kernel densities in the left column of Figures A.1, A.2. and A.3 of the Internet Appendix. We observe that the distributions for $M=6$ center around smaller out-of-sample variances and collapse more tightly around this value than the distributions for smaller values of $M$. This result holds for all averaging levels and supports the finding that combining all six strategies is beneficial when compared to averaging over a subset.
} 
In the context of our proposed rule, the previous findings demonstrate that averaging over a greater number of strategies is superior to averaging over fewer. This result holds irrespective of the averaging level as well as the estimation window. We further find that averaging over more strategies is not only beneficial with respect to the out-of-sample variance, but also in terms of the out-of-sample Sharpe ratio 8 Thus, for the rest of our paper we apply our rule to the Sample and the remaining five strategies in Panel B of Table 1, setting $M=6$.

\section{[TABLE 5 ABOUT HERE]}

We compare the out-of-sample variances of our averaging rule against the considered established strategies in Table 5. We observe that our rule achieves, on average, lower out-ofsample variances than the single strategies over all estimation windows. This finding again holds for all averaging levels. We note that the out-of-sample variances of our rule are, on average, lower than any of the sophisticated benchmarks in Panel C!9

\section{[TABLE 6 ABOUT HERE]}

We report the risk-adjusted performance of our rule in comparison to the established strategies in Table 6. We observe that AV-Wgt delivers, on average, slightly higher Sharpe ratios than AV-Inv and AV-Cov. The Sharpe ratios of AV-Wgt for $\tau=60$ and 120 are higher than the Sharpe ratios of any strategy in Panel B. We find that only SC delivers, on average, a higher Sharpe ratio for the remaining estimation windows. The average Sharpe ratios of AV-Wgt in comparison to the benchmarks in Panel C, are higher than those of the considered strategies across all estimation windows. These results suggest that our rule compares favorably to the

\footnotetext{
${ }^{8}$ We report the results for the average out-of-sample Sharpe ratio in Table A.1 of the Internet Appendix. We find that the average Sharpe ratio increases with the number of strategies. Investigating the distribution of the out-of-sample Sharpe ratios for AV-Wgt, AV-Inv, and AV-Cov using kernel densities, we turn to the right column of Figures A.1 A.2, and A.3 of the Internet Appendix. We find that the distributions for $M=6$ are centered around a higher mean value and collapse for estimation windows up to $\tau=120$ more tightly around the mean value. We observe that this effect diminishes as the estimation window increases.

${ }^{9}$ We investigate the out-of-sample variances of our rule in comparison to the existing strategies in more detail, exploring the kernel densities for AV-Wgt, AV-Inv, and AV-Cov in Figures A.4, A.5 and A.6 of the Internet Appendix. The left column in each figure shows the densities in comparison to the single strategies. We find that the distributions of $\mathrm{AV}-\mathrm{Wgt}, \mathrm{AV}-\mathrm{Inv}$, and $\mathrm{AV}$-Cov are centered around a smaller mean value and collapse more tightly relative to the aforementioned strategies for estimation windows up to $\tau=480$. The kernel densities of each averaging level of our rule also compare favorably to the benchmarks in the right column of the figures. We find that the distributions of AV-Wgt, AV-Inv, and AV-Cov collapse as tightly as the competing benchmarks, but around a smaller mean value. This observation holds for estimation windows of up to $\tau=480$.
} 
existing strategies not only with respect to the variance, but also in terms of the Sharpe ratio 10

Our simulation study has three key results: First, the contributions of the estimation errors to the out-of-sample variances are uncorrelated between the considered strategies, offering sizable diversification benefits. This suggests that averaging over a multitude of strategies reduces the overall impact of estimation errors. Second, combining more strategies yields a lower variance and a higher risk-adjusted performance. The performance across the averaging levels is similar, but slightly in favor of the portfolio weight level. We thus use AV-Wgt as the reference strategy in the empirical section of this paper. Third, we find that our rule compares favorably to the set of established strategies, suggesting that the combination of existing strategies represents an efficient way to alleviate the impact of estimation errors on out-of-sample performance.

\section{Empirical study}

\subsection{Data}

We evaluate the out-of-sample performance of our averaging rule in comparison to the eleven competing strategies on five empirical data sets based on daily excess returns 11 Table 7 provides an overview of the data sets and their source. We use the Fama-French six and 25-factor as well as the ten and 30-industry portfolios from Kenneth French's homepage. The construction of the Stock500 data set follows Chan et al. (1999), Jagannathan and Ma (2003), and DeMiguel et al. (2009b). In July of each year, we select the 500 largest stocks in terms of market capitalization from all NYSE, AMEX, and NASDAQ stocks in the Center for Research in Security Prices (CRSP) database that fulfill certain filter criteria. We filter out stocks with a price of less than $\$ 5$, as well as stocks with missing excess returns in the 756 trading days preceding, or the 252 days subsequent to the selection date. ${ }^{12}$ The selected stocks constitute the investment universe for one year, which is then revised based on the outlined criteria.

\footnotetext{
${ }^{10}$ The kernel densities of the out-of-sample Sharpe ratios for AV-Wgt, AV-Inv, and AV-Cov in Figures A.7. A.8 and A.9 of the Internet Appendix, corroborate the favorable comparison relative to the existing strategies. The left column of the aforementioned figures presents the results in comparison to the single strategies. We observe that the distribution of the out-of-sample Sharpe ratios for all averaging levels of our rule collapses more tightly around a higher mean value than for the aforementioned strategies, except SC. The right column of the figures shows the results in comparison to the benchmarks. We find that our rule centers for all estimation windows around a higher mean value and collapses more tightly around the respective mean for estimation windows up to $\tau=120$.

${ }^{11}$ Our choice of a daily data frequency follows Ledoit and Wolf (2017) and is also in-line with industry standards. We report empirical results on a monthly data frequency in Tables A.3 - A.6 of the Internet Appendix. We find that the results based on monthly excess returns are qualitatively similar to the ones presented in this section.

${ }^{12}$ We use the common convention that a calendar month comprises 21 trading days throughout the empirical section of this paper. The 252 and 756 trading days correspond to one and three years of data.
} 


\section{[TABLE 7 ABOUT HERE]}

We compute the out-of-sample excess returns for each portfolio strategy from Table 1 using the common rolling-sample procedure, setting $\tau=756$ observations. Starting in June 1973, we estimate the portfolio weights of each portfolio strategy using only the information in the estimation window comprising the most recent $\tau$ excess returns. We hold the estimated portfolio weights constant for 21 trading days, representing a one-month holding period, and save the corresponding out-of-sample excess returns. We then move the estimation window forward by 21 trading days and repeat the aforementioned procedure over the entire sample period.

\subsection{Performance evaluation}

We compare our averaging rule with each competing portfolio strategy in terms of variance $\left(s^{2}\right)$ and Sharpe ratio $(S R)$ of out-of-sample excess returns, the average portfolio turnover $(T R N)$, and the average short interest $(S I)$. The respective metrics for the $i$-th portfolio strategy are the same as used by DeMiguel et al. (2013) and given by:

$$
\begin{array}{r}
s_{i}^{2}=\frac{1}{T-\tau-1} \sum_{t=\tau}^{T-1}\left(\hat{w}_{i, t}^{\prime} r_{t+1}-\bar{r}_{i}\right)^{2}, \text { with } \bar{r}_{i}=\frac{1}{T-\tau} \sum_{t=\tau}^{T-1} \hat{w}_{i, t}^{\prime} r_{t+1}, \\
S R_{i}=\frac{\bar{r}_{i}}{s_{i}}, \\
T R N_{i}=\frac{1}{T-\tau-1} \sum_{t=1}^{(T-21) / 21}\left\|\hat{w}_{i, \tau+t \cdot 21+1}-\hat{w}_{i,(\tau+t \cdot 21)^{+}}\right\|_{1}, \\
S I_{i}=\frac{1}{T-\tau} \sum_{t=\tau}^{T} \frac{\left\|\hat{w}_{i, t}\right\|_{1}-1}{2},
\end{array}
$$

where $\hat{w}_{i, t}$ are the portfolio weights of strategy $i$ at time $t, \hat{w}_{i, t^{+}}$are the weights of rule $i$ at the end of $t$ but before rebalancing, $\hat{w}_{i, t+1}$ are the congruent weights after rebalancing at time $t+1$, and $\left\|\hat{w}_{t}\right\|_{1}$ is the 1-norm of the portfolio weights at time $t$. We further denote the vector of excess returns at $t+1$ by $r_{t+1}{ }^{13}$

We measure the statistical significance of the differences between our averaging rule on the portfolio weight level, AV-Wgt, and LWNLS when investigating the out-of-sample variance using robust inference proposed by Ledoit and Wolf (2011). We compare our rule with ID when evaluating the out-of-sample Sharpe ratio, using the robust procedure from Ledoit and Wolf

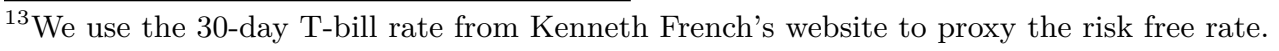


(2008). 14 We choose LWNLS as a benchmark for variance testing because it represents the most sophisticated strategy in terms of portfolio variance minimization. The ID portfolio serves as a benchmark for the Sharpe ratio because DeMiguel et al. (2009b) find that no sophisticated portfolio strategy outperforms the $1 / \mathrm{N}$ portfolio ${ }^{15}$

\subsection{Out-of-sample results}

Table 8 reports the out-of-sample variances of our proposed rule on the three averaging levels, the single strategies, and the benchmarks. For all considered data sets we observe that our rule achieves comparable variances across all averaging levels. 16 The variance of AV-Wgt is on all averaging levels and across all data sets lower than the variances of the single strategies, except the Sample strategy on the $25 \mathrm{FF}$ data set.

\section{[TABLE 8 ABOUT HERE]}

Comparing AV-Wgt to the benchmark strategies in Panel C, we find that AV-Wgt achieves on four out of five data sets lower variances than LWNLS, being significant on three out of five. In comparison to the linear shrinkage strategies LW1F and LWID, we find that AV-Wgt delivers on three, respectively four, data sets lower variances. The variance of AV-Wgt is on all levels and across all data sets lower than LWCC and KDM. Our results show that averaging over multiple strategies leads to a more efficient diversification and compares favorably to existing shrinkage approaches in terms of out-of-sample variance.

To study the relevance of each individual strategy within our AV-Wgt rule, we plot the estimated averaging weights for all data sets in Figure 2. We observe that AV-Wgt combines at least two estimators at any point in time across all data sets. The maximum number of strategies with positive weights amounts to four for the $6 \mathrm{FF}$, five for 30Ind and STOCK500, and all six strategies for the remaining data sets. The results confirm the relevance of each strategy within our averaging rule. Our rule is hereby not confined to combining (always) the sample with just one strategy.

\section{[FIGURE 2 ABOUT HERE]}

\footnotetext{
${ }^{14}$ We confine the statistical inference to the aforementioned pair-wise tests to avoid multiple-testing problems in the inference (see Ledoit and Wolf (2017) and references therein).

${ }^{15}$ Note that the minimum-variance portfolio based on a scalar multiple of the identity matrix corresponds to the $1 / \mathrm{N}$ portfolio.

${ }^{16}$ For the remaining performance metrics we also find similar results on the different averaging levels, and thus, confine the discussion to AV-Wgt vis-a-vis the single strategies and benchmarks for the sake of brevity.
} 
Table 9 shows the out-of-sample Sharpe ratios for our averaging rule and the various strategies. In Panel B, we observe that our rule delivers the second highest Sharpe ratio on the Fama-French and industry portfolios compared to the single strategies. The Sharpe ratio of AV-Wgt on the Stock500 data set is higher than for any strategy in Panel B. We observe that $\mathrm{AV}$-Wgt delivers on four out of five data sets a statistically significant Sharpe ratio increase relative to the ID strategy. The smallest increase in Sharpe ratio amounts to $25 \%$ for the 30Ind portfolio, being economically, but not statistically significant. Thus, we conclude that our averaging rule meets the challenge set forth by DeMiguel et al. (2009b) to deliver significantly higher risk-adjusted excess returns.

\section{[TABLE 9 ABOUT HERE]}

Comparing AV-Wgt to the Ledoit-Wolf shrinkage strategies LW1F, LWID, LWCC and LWNLS, we observe a comparable performance across all data sets. ${ }^{17}$ AV-Wgt performs somewhat better on the $6 \mathrm{FF}$ and 30Ind data set, while the shrinkage strategies seem to deliver slightly higher Sharpe ratios on the 10Ind and Stock500 data sets. The performance of AV-Wgt and the shrinkage strategies on the 10Ind data set is almost identical.

We assess the practicality of our averaging rule against the established strategies in terms of turnover and short interest. The results in Table 10 report that the average monthly turnover of $\mathrm{AV}$-Wgt is modest across all data sets and ranges from $4.721 \%$ on the 10 Ind to $16.520 \%$ on the $25 \mathrm{FF}$ data set. The turnover of AV-Wgt is similar to the Ledoit-Wolf shrinkage strategies on the Fama-French and industry portfolios, but only half the turnover of the aforementioned strategies on the Stock500 data set 18 The turnover of AV-Wgt is generally lower for all data sets in comparison to the single strategies allowing for short sales, i.e. Sample, 1F, CC and POET.

\section{[TABLE 10 ABOUT HERE]}

As shown in Table 11, the short interest of our averaging rule ranges between $45.509 \%$ on the $10 \mathrm{Ind}$ and $201.652 \%$ on the $25 \mathrm{FF}$ data set. On average, the reported short interest of

\footnotetext{
${ }^{17}$ We also observe that the benchmarks in Panel C generate higher Sharpe ratios than the ID portfolio, which is in line with the results of Ledoit and Wolf (2017).

${ }^{18}$ The Fama-French and industry portfolios are constructed at the end of each June and its composition is kept stable for the next 12 months until revision of the applicable investment universe. Next June the composition changes, stocks no longer matching the criteria are dropped, and new stocks are included in the portfolios. This cannot be accounted for during the turnover computation and, consequently, we apply the same logic to the CRSP stock data set, i.e. the replacement of a stock in the investment universe is not considered for the turnover.
} 
AV-Wgt is lower than that of the Ledoit-Wolf shrinkage strategies. The favorable comparison is mainly due to the Stock500 data set, where AV-Wgt compared to LWNLS and LWID realizes a short interest reduction between 10 and $60 \%$. The short interest of our rule is, on average, slightly higher than for the strategies that allow for short sales, except for the Sample and POET strategy.

[TABLE 11 ABOUT HERE]

Concluding our empirical analysis, we find that our averaging rule delivers significantly lower out-of-sample variances than established estimators of the global minimum-variance portfolio. This result is important because the out-of-sample variance is the primary performance criterion when estimating a minimum-variance portfolio (see Ledoit and Wolf 2017). The risk-adjusted performance of our averaging rule is significantly higher in comparison to the $1 / \mathrm{N}$ portfolio and is akin to the Ledoit-Wolf shrinkage strategies, including the non-linear estimator of Ledoit and Wolf (2017). The modest turnover, especially on the Stock500 data set, compares favorably to existing strategies and supports the practicality of our rule.

\section{Conclusion}

In this paper, we propose a novel approach to alleviate the impact of estimation errors on out-of-sample portfolio performance. Our approach builds on the idea of shrinkage and consists of combining the Sample with a multitude of structured estimators to minimize the expected out-of-sample variance of the corresponding minimum-variance portfolio. The motivation behind this idea is to bring the principle of diversification from the portfolio to the estimation level. If errors in the individual estimators are not perfectly correlated, the combination of the latter offers diversification benefits. Consequently, the resulting estimates of the minimum-variance portfolio weights from our averaging procedure should be less error prone and, on average, deliver an improved out-of-sample performance.

We evaluate our rule through extensive simulations and find that averaging over a greater number of estimators is superior to averaging over fewer in terms of out-of-sample variance and Sharpe ratio. This observation holds for all possible averaging levels, i.e. the portfolio weight, the inverse, and the covariance level. We attribute the performance gains to the leveraged benefits of estimation error diversification between the considered estimators. This diversification 
potential exists for large and small estimation windows. Our rule achieves, on average, lower out-of-sample variances than any of the competing eleven minimum-variance strategies. The results for the risk-adjusted performance are similar for small estimation windows, suggesting that our averaging rule alleviates the impact of estimation errors on out-of-sample portfolio performance.

Our empirical results are in line with the findings from the simulation study. Our proposed averaging rule achieves a lower out-of-sample portfolio variance than any single strategy and compares favorably to the sophisticated non-linear shrinkage approach of Ledoit and Wolf (2017). In terms of out-of-sample Sharpe ratio, we find that our averaging rule outperforms the 1/N portfolio on all data sets, with significant increases on four out of five. In terms of turnover and short interest, the portfolio weights of our rule are smooth over time and do not contain extreme positions.

Our results suggest that the diversification of estimation errors through averaging over the sample and multiple structured estimators provides a fruitful way to reduce the impact of estimation errors on out-of-sample portfolio performance. Future research may elaborate on alternative approaches for determining averaging weights, or may extend the set of estimators used in the averaging procedure to explore potential limits of the suggested estimation error diversification. 


\section{References}

Britten-Jones, M. (1999). The sampling error in estimates of mean-variance efficient portfolio weights. Journal of Finance, 54(2):655-671.

Brodie, J., Daubechies, I., De Mol, C., Giannone, D., and Loris, I. (2009). Sparse and stable Markowitz portfolios. Proceedings of the National Academy of Sciences of the United States of America, 106(30):12267-12272.

Chan, L. K., Karceski, J., and Lakonishok, J. (1999). On portfolio optimization: Forecasting covariances and choosing the risk model. Review of Financial Studies, 12(5):937-974.

DeMiguel, V., Garlappi, L., Nogales, F. J., and Uppal, R. (2009a). A generalized approach to portfolio optimization: Improving performance by constraining portfolio norms. Management Science, 55(5):798-812.

DeMiguel, V., Garlappi, L., and Uppal, R. (2009b). Optimal versus naive diversification: How inefficient is the 1/N portfolio strategy? Review of Financial Studies, 22(5):1915-1953.

DeMiguel, V., Martin-Utrera, A., and Nogales, F. J. (2013). Size matters: Optimal calibration of shrinkage estimators for portfolio selection. Journal of Banking and Finance, 37(8):3018-3034.

Elton, E. J. and Gruber, M. J. (1973). Estimating the Dependence Structure of Share Prices Implications for Portfolio Selection. The Journal of Finance, 28(5):1203-1232.

Fan, J., Liao, Y., and Mincheva, M. (2013). Large covariance estimation by thresholding principal orthogonal complements. Journal of the Royal Statistical Society. Series B: Statistical Methodology, 75(4):603-680.

Frost, P. A. and Savarino, J. E. (1986). An Empirical Bayes Approach to Efficient Portfolio Selection. Journal of Financial and Quantitative Analysis, 21(3):293.

Hansen, B. E. (2008). Least-squares forecast averaging. Journal of Econometrics, 146(2):342-350.

Jagannathan, R. and Ma, T. (2003). Risk Reduction in Large Portfolios: Why Imposing the Wrong Constraints Helps.

Kan, R. and Zhou, G. (2007). Optimal Portfolio Choice with Parameter Uncertainty. Journal of Financial and Quantitative Analysis, 42(3):621-656.

Kourtis, A., Dotsis, G., and Markellos, R. N. (2012). Parameter uncertainty in portfolio selection: Shrinking the inverse covariance matrix. Journal of Banking and Finance, 36(9):2522-2531.

Ledoit, O. and Wolf, M. (2003). Improved estimation of the covariance matrix of stock returns with an application to portfolio selection. Journal of Empirical Finance, 10(5):603-621.

Ledoit, O. and Wolf, M. (2004a). A well-conditioned estimator for large-dimensional covariance matrices. Journal of Multivariate Analysis, 88(2):365-411. 
Ledoit, O. and Wolf, M. (2004b). Honey, I shrunk the sample covariance matrix. Journal of Portfolio Management, 30(4):110-119+7.

Ledoit, O. and Wolf, M. (2008). Robust performance hypothesis testing with the Sharpe ratio. Journal of Empirical Finance, 15(5):850-859.

Ledoit, O. and Wolf, M. (2011). Robust Performances Hypothesis Testing With the Variance. Wilmott, 2011(55):86-89.

Ledoit, O. and Wolf, M. (2017). Nonlinear shrinkage of the covariance matrix for portfolio selection: Markowitz meets goldilocks. Review of Financial Studies, 30(12):4349-4388.

Li, J. (2015). Sparse and Stable Portfolio Selection With Parameter Uncertainty. Journal of Business and Economic Statistics, 33(3):381-392.

Merton, R. C. (1980). On estimating the expected return on the market: An exploratory investigation. Topics in Catalysis, 8(4):323-361.

Sharpe, W. F. (1963). A Simplified Model for Portfolio Analysis. Management Science, 9(2):277293.

Sharpe, W. F. (1967). Portfolio Analysis. Journal of Financial and Quantitative Analysis, $2(2): 76$.

Shi, F., Shu, L., Yang, A., and He, F. (2020). Improving minimum-variance portfolios by alleviating overdispersion of eigenvalues. Journal of Financial and Quantitative Analysis, $55(8): 2700-2731$.

Stein, C. (1975). Estimation of a covariance matrix. Rietz Lecture, 1:1-6.

Stein, C. (1986). Lectures on the theory of estimation of many parameters. Journal of Soviet Mathematics, 34(1):1373-1403.

Timmermann, A. (2006). Chapter 4 Forecast Combinations. In Handbook of Economic Forecasting, pages $135-196$.

Tu, J. and Zhou, G. (2011). Markowitz meets Talmud: A combination of sophisticated and naive diversification strategies. Journal of Financial Economics, 99(1):204-215.

Xing, X., Hu, J., and Yang, Y. (2014). Robust minimum variance portfolio with L-infinity constraints. Journal of Banking and Finance, 46(1):107-117.

Yen, Y. M. (2016). Sparse Weighted-Norm Minimum Variance Portfolios. Review of Finance, 20(3):1259-1287. 


\section{Figures and tables}

Table 1: List of averaging rule, single strategies, and benchmarks

This table lists the proposed averaging rule on the considered levels in Panel A, the single strategies in Panel B, and the benchmarks, representing sophisticated estimates of the minimumvariance portfolio, in Panel C. The last column of the table shows the abbreviation that we use to refer to the respective averaging rule or strategy. We do not include the $1 / \mathrm{N}$ strategy from DeMiguel et al. (2009b) separately because ID corresponds to the $1 / \mathrm{N}$ strategy.

\begin{tabular}{|c|c|c|}
\hline$\#$ & Description & Abbreviation \\
\hline \multicolumn{3}{|c|}{ Panel A: Averaging rule across three levels } \\
\hline 1 & Averaging rule on the portfolio weight level & $\mathrm{AV}-\mathrm{Wgt}$ \\
\hline 2 & Averaging rule on the inverse covariance level & AV-Inv \\
\hline 3 & Averaging rule on the covariance level & $\mathrm{AV}-\mathrm{Cov}$ \\
\hline \multicolumn{3}{|c|}{ Panel B: Single strategies } \\
\hline 4 & $\begin{array}{l}\text { Minimum-variance portfolio without constraints based on the sample } \\
\text { covariance matrix }\end{array}$ & Sample \\
\hline 5 & $\begin{array}{l}\text { Minimum-variance portfolio with the market as a single factor Sharpe } \\
\text { 1963) }\end{array}$ & $1 \mathrm{~F}$ \\
\hline 6 & $\begin{array}{l}\text { Minimum-variance portfolio with the constant correlation model implied } \\
\text { covariance matrix (Elton and Gruber, } 1973)\end{array}$ & $\mathrm{CC}$ \\
\hline 7 & $\begin{array}{l}\text { Minimum-variance portfolio with a scalar multiple of the identity covari- } \\
\text { ance matrix }\end{array}$ & ID \\
\hline 8 & $\begin{array}{l}\text { Minimum-variance portfolio with short-sale constraints Jagannathan } \\
\text { and Ma, 2003) }\end{array}$ & $\mathrm{SC}$ \\
\hline 9 & $\begin{array}{l}\text { Minimum-variance portfolio based on the principal components of the } \\
\text { sample covariance matrix (Fan et al., 2013) }\end{array}$ & POET \\
\hline \multicolumn{3}{|c|}{ Panel C: Benchmarks } \\
\hline 10 & $\begin{array}{l}\text { Minimum-variance portfolio with the covariance matrix as weighted } \\
\text { average between the sample covariance and the single factor covariance } \\
\text { matrix (Ledoit and Wolf }, 2003)\end{array}$ & LW1F \\
\hline 11 & $\begin{array}{l}\text { Minimum-variance portfolio with the covariance matrix as weighted } \\
\text { average between the sample covariance and a scalar multiple of the } \\
\text { identity matrix (Ledoit and Wolf }, \text { 2004a })\end{array}$ & LWID \\
\hline 12 & $\begin{array}{l}\text { Minimum-variance portfolio with the covariance matrix as weighted aver- } \\
\text { age between the sample covariance and a constant correlation covariance } \\
\text { matrix (Ledoit and Wolf }, 2004 \mathrm{~b})\end{array}$ & LWCC \\
\hline 13 & $\begin{array}{l}\text { Minimum-variance portfolio with the non-linear shrinkage implied covari- } \\
\text { ance matrix (Ledoit and Wolf, 2017) }\end{array}$ & LWNLS \\
\hline 14 & Three-fund strategy (Kourtis et al. 2012$)$ & KDM \\
\hline
\end{tabular}


Table 2: Summary statistics of simulation parameters

This table reports the monthly means, standard deviations, and cross-correlations between the three Fama-French factors market (MKT), size (SMB), and value (HML) in the simulation. The reported values correspond to the empirical values over the period from 06/1963-08/2007. The time horizon for the calibration of the parameters corresponds to Tu and Zhou (2011).

\begin{tabular}{lccc}
\hline & MKT & SMB & HML \\
\hline Mean & 0.478 & 0.236 & 0.450 \\
Standard Deviation & 4.379 & 3.215 & 2.809 \\
Cross-correlations & & & \\
MKT & 1.000 & 0.287 & -0.390 \\
SMB & & 1.000 & -0.260 \\
HML & & & 1.000 \\
\hline
\end{tabular}


Table 3: Descriptive statistics of estimation errors across estimation windows

This table shows the means, standard deviations (Std), and Bravais-Pearson correlation coefficients of the estimation errors between the single strategies in Panel B of Table 1 . The estimation error for the $m$-th portfolio strategy in the $k$-th simulation run is defined as the following loss: $L_{k, m}=\left(\hat{w}_{k, m}-w_{k}\right)^{\prime} \Sigma_{k}\left(\hat{w}_{k, m}-w_{k}\right)$, where $\hat{w}_{i, k}$ are the estimated minimum-variance portfolio weights and $w_{k}$ are the true minimum-variance portfolio weights, which are computed using the population covariance matrix of the respective run, $\Sigma_{k}$. The reported values show the correlation over 10,000 simulation runs between the losses of the respective estimators. Panels A - E report the respective results for estimation windows of $\tau=\{60,120,240,480,960\}$. The reported means and standard deviations are scaled by $10 \mathrm{e}+03$, respectively.

\begin{tabular}{lcccccc}
\hline & Sample & $1 \mathrm{~F}$ & $\mathrm{CC}$ & $\mathrm{ID}$ & $\mathrm{SC}$ & POET \\
\hline Panel A: & Estimation window & $\tau=\mathbf{6 0}$ & & & & \\
Mean & 1.326 & 0.440 & 0.537 & 0.940 & 0.441 & 0.548 \\
Std & 0.524 & 0.148 & 0.193 & 0.118 & 0.150 & 0.169
\end{tabular}

\section{Cross-correlations}

\begin{tabular}{|c|c|c|c|c|c|c|}
\hline Sample & 1.000 & 0.365 & 0.224 & -0.171 & 0.259 & 0.434 \\
\hline $1 \mathrm{~F}$ & & 1.000 & 0.357 & -0.174 & 0.139 & 0.745 \\
\hline $\mathrm{CC}$ & & & 1.000 & -0.345 & 0.349 & 0.413 \\
\hline ID & & & & 1.000 & -0.086 & -0.257 \\
\hline $\mathrm{SC}$ & & & & & 1.000 & 0.347 \\
\hline POET & & & & & & 1.000 \\
\hline \multicolumn{7}{|c|}{ Panel B: Estimation window $\tau=120$} \\
\hline Mean & 0.481 & 0.394 & 0.480 & 0.940 & 0.306 & 0.402 \\
\hline Std & 0.159 & 0.128 & 0.166 & 0.118 & 0.092 & 0.115 \\
\hline
\end{tabular}

\section{Cross-correlations}

\begin{tabular}{|c|c|c|c|c|c|c|}
\hline Sample & 1.000 & 0.216 & 0.180 & -0.209 & 0.347 & 0.418 \\
\hline $1 \mathrm{~F}$ & & 1.000 & 0.106 & -0.024 & -0.043 & 0.609 \\
\hline $\mathrm{CC}$ & & & 1.000 & -0.370 & 0.327 & 0.232 \\
\hline ID & & & & 1.000 & -0.060 & -0.189 \\
\hline $\mathrm{SC}$ & & & & & 1.000 & 0.191 \\
\hline POET & & & & & & 1.000 \\
\hline \multicolumn{7}{|c|}{ Panel C: Estimation window $\tau=\mathbf{2 4 0}$} \\
\hline Mean & 0.209 & 0.376 & 0.453 & 0.940 & 0.228 & 0.291 \\
\hline Std & 0.066 & 0.120 & 0.151 & 0.118 & 0.066 & 0.088 \\
\hline \multicolumn{7}{|c|}{ Cross-correlations } \\
\hline Sample & 1.000 & 0.103 & 0.140 & -0.220 & 0.282 & 0.334 \\
\hline $1 \mathrm{~F}$ & & 1.000 & -0.025 & 0.106 & -0.112 & 0.492 \\
\hline $\mathrm{CC}$ & & & 1.000 & -0.388 & 0.344 & 0.097 \\
\hline ID & & & & 1.000 & 0.013 & -0.048 \\
\hline $\mathrm{SC}$ & & & & & 1.000 & 0.053 \\
\hline POET & & & & & & 1.000 \\
\hline
\end{tabular}

Table continues on next page 
Table 3 continues here

\begin{tabular}{|c|c|c|c|c|c|c|}
\hline & Sample & $1 \mathrm{~F}$ & $\mathrm{CC}$ & ID & $\mathrm{SC}$ & POET \\
\hline \multicolumn{7}{|c|}{ Panel D: Estimation window $\tau=480$} \\
\hline Mean & 0.099 & 0.369 & 0.438 & 0.940 & 0.185 & 0.212 \\
\hline Std & 0.030 & 0.113 & 0.145 & 0.118 & 0.056 & 0.072 \\
\hline \multicolumn{7}{|c|}{ Cross-correlations } \\
\hline Sample & 1.000 & 0.024 & 0.117 & -0.210 & 0.162 & 0.225 \\
\hline $1 \mathrm{~F}$ & & 1.000 & -0.058 & 0.194 & -0.101 & 0.391 \\
\hline $\mathrm{CC}$ & & & 1.000 & -0.380 & 0.323 & 0.014 \\
\hline ID & & & & 1.000 & 0.109 & 0.090 \\
\hline $\mathrm{SC}$ & & & & & 1.000 & -0.023 \\
\hline POET & & & & & & 1.000 \\
\hline \multicolumn{7}{|c|}{ Panel E: Estimation window $\tau=960$} \\
\hline Mean & 0.049 & 0.366 & 0.432 & 0.940 & 0.162 & 0.164 \\
\hline Std & 0.015 & 0.109 & 0.140 & 0.118 & 0.053 & 0.059 \\
\hline \multicolumn{7}{|c|}{ Cross-correlations } \\
\hline Sample & 1.000 & -0.026 & 0.114 & -0.238 & 0.077 & 0.141 \\
\hline $1 \mathrm{~F}$ & & 1.000 & -0.057 & 0.226 & -0.113 & 0.349 \\
\hline $\mathrm{CC}$ & & & 1.000 & -0.392 & 0.308 & -0.027 \\
\hline ID & & & & 1.000 & 0.158 & 0.180 \\
\hline $\mathrm{SC}$ & & & & & 1.000 & -0.007 \\
\hline POET & & & & & & 1.000 \\
\hline
\end{tabular}


Table 4: Simulation-based average out-of-sample variances for a varying number of strategies

This table reports the average out-of-sample variances of our proposed averaging rule on the portfolio weight (AV-Wgt), the inverse (AV-Inv), and the covariance (AV-Cov) level over 10,000 simulation runs for a varying number of single strategies, $M$. The out-of-sample variance in the $k$-th simulation run is computed as $\hat{\sigma}_{k}^{2}=\hat{w}_{k, m}^{\prime} \Sigma_{k} \hat{w}_{k, m}$, where $\Sigma_{k}$ is the population covariance matrix resulting from the random pairing of the assets' factor loadings, their mispricing, as well as their idiosyncratic volatility. $\hat{w}_{k, m}$ are the portfolio weights of the $m$-th strategy based on the $\tau$ simulated returns. Panels A-E show the respective weights for the estimation windows of $\tau=\{60,120,240,480,960\}$. The results for $M=2$ up to $M=6$ represent averages over all possible permutations from combining the Sample with the five remaining strategies from Panel $\mathrm{B}$ in Table 1. The reported average out-of-sample variances are scaled by $10 \mathrm{e}+03$.

\begin{tabular}{lccccc}
\hline & $M=2$ & $M=3$ & $M=4$ & $M=5$ & $M=6$ \\
\hline Panel A: Estimation window & $\tau=\mathbf{6 0}$ & & & \\
AV-Wgt & 2.400 & 2.281 & 2.232 & 2.191 & 2.190 \\
AV-Inv & 2.399 & 2.279 & 2.232 & 2.190 & 2.189 \\
AV-Cov & 2.347 & 2.289 & 2.261 & 2.240 & 2.237
\end{tabular}

Panel B: Estimation window $\tau=\mathbf{1 2 0}$

$\begin{array}{llllll}\text { AV-Wgt } & 2.200 & 2.134 & 2.097 & 2.063 & 2.062 \\ \text { AV-Inv } & 2.201 & 2.134 & 2.097 & 2.064 & 2.063 \\ \text { AV-Cov } & 2.170 & 2.131 & 2.107 & 2.091 & 2.089\end{array}$

Panel C: Estimation window $\tau=\mathbf{2 4 0}$

$\begin{array}{llllll}\text { AV-Wgt } & 2.052 & 2.030 & 2.013 & 1.995 & 1.994 \\ \text { AV-Inv } & 2.052 & 2.030 & 2.014 & 1.995 & 1.994 \\ \text { AV-Cov } & 2.044 & 2.028 & 2.016 & 2.007 & 2.006\end{array}$

Panel D: Estimation window $\tau=480$

$\begin{array}{llllll}\text { AV-Wgt } & 1.967 & 1.960 & 1.955 & 1.949 & 1.948 \\ \text { AV-Inv } & 1.967 & 1.960 & 1.955 & 1.949 & 1.948 \\ \text { AV-Cov } & 1.969 & 1.963 & 1.960 & 1.956 & 1.956\end{array}$

Panel E: Estimation window $\tau=\mathbf{9 6 0}$

\begin{tabular}{llllll} 
AV-Wgt & 1.925 & 1.923 & 1.922 & 1.920 & 1.920 \\
AV-Inv & 1.925 & 1.923 & 1.922 & 1.920 & 1.920 \\
AV-Cov & 1.925 & 1.923 & 1.922 & 1.921 & 1.921 \\
\hline
\end{tabular}


Table 5: Simulation-based average out-of-sample variances for the averaging rule, strategies, and benchmarks

This table reports the average out-of-sample variances of our proposed averaging rule on the portfolio weight (AV-Wgt), the inverse (AV-Inv), and the covariance (AV-Cov) level over 10,000 simulation runs in comparison to established strategies. The out-of-sample variance in the $k$-th simulation run is computed as $\hat{\sigma}_{k}^{2}=\hat{w}_{k, m}^{\prime} \Sigma_{k} \hat{w}_{k, m}$, where $\Sigma_{k}$ is the population covariance matrix resulting from the random pairing of the assets' factor loadings, their mispricing, as well as their idiosyncratic volatility. $\hat{w}_{k, m}$ are the portfolio weights of the $m$-th strategy based on the $\tau$ simulated returns. The reported variances for the considered estimation windows of $\tau=\{60,120,240,480,960\}$ are scaled by $10 \mathrm{e}+03$. The results for AV-Wgt, AV-Inv, and AV-Cov are based on averaging over all $M=6$ strategies from Panel B in Table 1 .

\begin{tabular}{|c|c|c|c|c|c|}
\hline & $\tau=60$ & $\tau=120$ & $\tau=240$ & $\tau=480$ & $\tau=960$ \\
\hline \multicolumn{6}{|c|}{ Panel A: Averaging rule across three levels } \\
\hline $\mathrm{AV}-\mathrm{Wgt}$ & 2.190 & 2.062 & 1.994 & 1.948 & 1.920 \\
\hline AV-Inv & 2.189 & 2.063 & 1.994 & 1.948 & 1.920 \\
\hline $\mathrm{AV}-\mathrm{Cov}$ & 2.237 & 2.089 & 2.006 & 1.956 & 1.921 \\
\hline \multicolumn{6}{|c|}{ Panel B: Single strategies } \\
\hline Sample & 3.203 & 2.357 & 2.085 & 1.975 & 1.927 \\
\hline $1 \mathrm{~F}$ & 2.316 & 2.271 & 2.251 & 2.244 & 2.244 \\
\hline $\mathrm{CC}$ & 2.413 & 2.357 & 2.329 & 2.313 & 2.311 \\
\hline ID & 2.816 & 2.816 & 2.816 & 2.816 & 2.816 \\
\hline $\mathrm{SC}$ & 2.317 & 2.183 & 2.103 & 2.060 & 2.040 \\
\hline POET & 2.424 & 2.290 & 2.167 & 2.088 & 2.042 \\
\hline \multicolumn{6}{|c|}{ Panel C: Benchmarks } \\
\hline LW1F & 2.276 & 2.141 & 2.034 & 1.963 & 1.924 \\
\hline LWID & 2.474 & 2.225 & 2.057 & 1.968 & 1.925 \\
\hline LWCC & 2.315 & 2.165 & 2.044 & 1.966 & 1.925 \\
\hline LWNLS & 2.338 & 2.166 & 2.041 & 1.965 & 1.926 \\
\hline $\mathrm{KDM}$ & 2.316 & 2.271 & 2.251 & 2.244 & 2.244 \\
\hline
\end{tabular}


Table 6: Simulation-based average out-of-sample Sharpe ratios for the averaging rule, strategies, and benchmarks

This table reports the average out-of-sample Sharpe ratios of our proposed averaging rule on the portfolio weight (AV-Wgt), the inverse (AV-Inv), and the covariance (AV-Cov) level over 10,000 simulation runs in comparison to established portfolio strategies. The out-of-sample Sharpe ratio in the $k$-th simulation run is computed as $\hat{\Psi}_{k}=\left(\hat{w}_{k, m}^{\prime} \mu_{k}\right)\left(\hat{w}_{k, m} \Sigma_{k} \hat{w}_{k, m}\right)^{-1 / 2}$, where $\mu_{k}$ is the population mean vector, and $\Sigma_{k}$ is the population covariance matrix resulting from the random pairing of the assets' factor loadings, their mispricing, as well as their idiosyncratic volatility. $\hat{w}_{k, m}$ are the estimated portfolio weights of the $m$-th strategy based on the $\tau$ simulated returns. The reported Sharpe ratios for the considered estimation windows of $\tau=\{60,120,240,480,960\}$ are scaled by $10 \mathrm{e}+03$. The results for AV-Wgt, AV-Inv, and AV-Cov are based on averaging over all $M=6$ strategies from Panel B in Table 1 .

\begin{tabular}{|c|c|c|c|c|c|}
\hline & $\tau=60$ & $\tau=120$ & $\tau=240$ & $\tau=480$ & $\tau=960$ \\
\hline \multicolumn{6}{|c|}{ Panel A: Averaging rule } \\
\hline AV-Wgt & 140.320 & 142.616 & 143.620 & 143.577 & 143.478 \\
\hline AV-Inv & 140.341 & 142.571 & 143.580 & 143.547 & 143.460 \\
\hline AV-Cov & 139.006 & 141.766 & 143.051 & 143.410 & 143.230 \\
\hline \multicolumn{6}{|c|}{ Panel B: Single Portfolio Strategies } \\
\hline Sample & 110.089 & 127.257 & 135.407 & 138.937 & 140.939 \\
\hline $1 \mathrm{~F}$ & 128.728 & 127.730 & 126.888 & 126.411 & 126.456 \\
\hline $\mathrm{CC}$ & 134.878 & 135.983 & 136.395 & 136.773 & 136.936 \\
\hline ID & 136.063 & 136.063 & 136.063 & 136.063 & 136.063 \\
\hline $\mathrm{SC}$ & 139.594 & 142.490 & 144.272 & 145.157 & 145.734 \\
\hline POET & 129.419 & 132.886 & 136.204 & 138.706 & 140.071 \\
\hline \multicolumn{6}{|c|}{ Panel C: Benchmarks } \\
\hline LW1F & 131.854 & 134.464 & 137.395 & 139.498 & 141.087 \\
\hline LWID & 129.352 & 133.675 & 137.726 & 139.910 & 141.370 \\
\hline LWCC & 134.884 & 136.993 & 139.235 & 140.677 & 141.751 \\
\hline LWNLS & 130.462 & 132.675 & 134.965 & 136.282 & 137.310 \\
\hline KDM & 128.728 & 127.730 & 126.888 & 126.411 & 126.456 \\
\hline
\end{tabular}


Table 7: List of the empirical data sets for daily data

This table lists the data sets for the empirical evaluation of our proposed averaging rule in comparison to existing portfolio strategies, their abbreviations, the number of assets in each data set, and the data sources. All data sets span the period 06/1973 - 06/2019, constitute daily data, and apply in the case of portfolio data the value weighting scheme to the respective constituents. Data from Kenneth French is taken from his website (http://mba.tuck.dartmouth. edu/pages/faculty/ken.french/data_library.html) and represents different cuts of the U.S. stock market. The STOCK500 data set contains the 500 largest single stocks in terms of market capitalization in July of every year after filtering out stocks that have a price of less than $\$ 5$, or exhibit missing returns in the preceding 756 and subsequent 252 trading days to the selection date. All stock prices are taken from the Center of Research in Security Prices (CRSP).

\begin{tabular}{llccc}
\hline$\#$ & Data set & Abbreviation & N & Source \\
\hline 1 & $\begin{array}{l}\text { 6 Fama and French portfolios of firms sorted by size } \\
\text { and book-to-market }\end{array}$ & $6 \mathrm{FF}$ & 6 & K. French \\
2 & $\begin{array}{l}25 \text { Fama and French portfolios of firms sorted by size } \\
\text { and book-to-market }\end{array}$ & $25 \mathrm{FF}$ & 25 & K. French \\
3 & $\begin{array}{l}10 \text { industry portfolios representing the U.S. stock mar- } \\
\text { ket }\end{array}$ & $10 \mathrm{Ind}$ & 10 & K. French \\
4 & $\begin{array}{l}30 \text { industry portfolios representing the U.S. stock mar- } \\
\text { ket }\end{array}$ & $30 I n d$ & 30 & K. French \\
5 & 500 Stocks with the largest market capitalization & STOCK500 & 500 & CRSP \\
\hline
\end{tabular}


Table 8: Daily out-of-sample variances in empirical data sets

This table reports the daily out-of-sample variances of our averaging rule on the portfolio weight (AV-Wgt), the inverse (AV-Inv), and the covariance (AV-Cov) level in Panel A, of the single strategies in Panel B, and of the benchmarks in Panel C. The results are shown for the Fama-French factor-mimicking and industry portfolios, as well as the STOCK500 data set. The out-of-sample period is from 06/1976 - 06/2019. The reported variances are scaled by $10 \mathrm{e}+03$. The abbreviations for the strategies in Panels B and $\mathrm{C}$, as well as for the data sets, are reported in Tables 1 and 7, respectively. We report statistical significance for the null hypothesis wherein the log out-of-sample variance of $\mathrm{AV}-\mathrm{Wgt}$ is greater than that of the non-linear shrinkage approach of Ledoit and Wolf (2017), LWNLS: $H_{0}: \log \left(\hat{\sigma}_{A V-W g t}^{2}\right)-\log \left(\hat{\sigma}_{L W N L S}^{2}\right) \leq 0$. We follow Ledoit and Wolf (2011) and use their proposed bootstrap procedure, using a block length of 5 and 1,000 iterations. Statistical significance at the 1, 5, and $10 \%$ level is denoted by ***, **, and *, respectively.

\begin{tabular}{lccccr}
\hline & $6 \mathrm{FF}$ & $25 \mathrm{FF}$ & 10Ind & 30Ind & STOCK500 \\
\hline Panel A: & Averaging rule & & & & \\
AV-Wgt & 0.5732 & 0.3802 & 0.5233 & 0.4489 & 0.2887 \\
AV-Inv & 0.5737 & 0.3806 & 0.5237 & 0.4491 & 0.2897 \\
AV-Cov & 0.5724 & 0.3789 & 0.5233 & 0.4517 & 0.2714
\end{tabular}

Panel B: Single strategies

$\begin{array}{llllll}\text { Sample } & 0.5742 & 0.3773 & 0.5305 & 0.4554 & 0.5313 \\ 1 \mathrm{~F} & 0.7787 & 0.7313 & 0.5742 & 0.5654 & 0.5593 \\ \text { CC } & 0.7340 & 0.7903 & 0.5755 & 0.6424 & 0.8001 \\ \text { ID } & 1.0892 & 1.0908 & 0.9686 & 1.0537 & 1.1287 \\ \text { SC } & 0.7700 & 0.7072 & 0.5812 & 0.5415 & 0.4192 \\ \text { POET } & 0.5937 & 0.4056 & 0.5463 & 0.5032 & 0.3247\end{array}$

Panel C: Benchmarks

\begin{tabular}{lrrrrr} 
LW1F & 0.5720 & 0.3769 & 0.5297 & 0.4536 & 0.2910 \\
LWID & 0.5820 & 0.3766 & 0.5302 & 0.4535 & 0.3648 \\
LWCC & 0.5874 & 0.4046 & 0.5321 & 0.4530 & 0.3219 \\
LWNLS & 0.5745 & 0.3758 & $0.5289^{* * *}$ & $0.4530^{* * *}$ & $0.3328^{* * *}$ \\
KDM & 0.7787 & 0.7313 & 0.5742 & 0.5654 & 0.5593 \\
\hline
\end{tabular}


Table 9: Daily out-of-sample Sharpe ratios in empirical data sets

This table reports the daily out-of-sample Sharpe ratios of our averaging rule on the portfolio weight (AV-Wgt), the inverse (AV-Inv), and the covariance (AV-Cov) level in Panel A, of the single strategies in Panel B, and of the benchmarks in Panel C. The results are shown for to the Fama-French factor-mimicking and industry portfolios, as well as the STOCK500 data set. The out-of-sample period is from 06/1976 - 06/2019. The abbreviations for the strategies in Panels $\mathrm{B}$ and $\mathrm{C}$, as well as for the data sets, are reported in Tables 1 and 7 , respectively. We use the 30-day T-bill rate as the risk-free rate. We report statistical significance for the null hypothesis wherein the Sharpe ratio of $\mathrm{AV}-\mathrm{Wgt}$ is greater than that of the $1 / \mathrm{N}$ portfolio, corresponding to the ID strategy: $H_{0}: S R_{A V-W g t}-S R_{I D} \leq 0$. We follow Ledoit and Wolf (2008) and use their proposed bootstrap procedure, using a block length of 5 and 1,000 iterations. Statistical significance at the 1, 5 and $10 \%$ level is denoted by $* * *, * *$, and ${ }^{*}$, respectively.

\begin{tabular}{|c|c|c|c|c|c|}
\hline & $6 \mathrm{FF}$ & $25 \mathrm{FF}$ & 10Ind & 30Ind & STOCK500 \\
\hline \multicolumn{6}{|c|}{ Panel A: Averaging rule } \\
\hline $\mathrm{AV}-\mathrm{Wgt}$ & 0.0770 & 0.0614 & 0.0471 & 0.0401 & 0.0509 \\
\hline AV-Inv & 0.0769 & 0.0615 & 0.0471 & 0.0399 & 0.0499 \\
\hline AV-Cov & 0.0760 & 0.0610 & 0.0468 & 0.0399 & 0.0555 \\
\hline \multicolumn{6}{|c|}{ Panel B: Single strategies } \\
\hline Sample & 0.0771 & 0.0641 & 0.0464 & 0.0376 & 0.0422 \\
\hline $1 \mathrm{~F}$ & 0.0606 & 0.0452 & 0.0498 & 0.0398 & 0.0428 \\
\hline $\mathrm{CC}$ & 0.0624 & 0.0460 & 0.0451 & 0.0391 & 0.0331 \\
\hline ID & $0.0343^{* * *}$ & $0.0189^{* * *}$ & $0.0332^{*}$ & 0.0320 & $0.0363^{*}$ \\
\hline $\mathrm{SC}$ & 0.0507 & 0.0300 & 0.0418 & 0.0404 & 0.0503 \\
\hline POET & 0.0806 & 0.0546 & 0.0444 & 0.0373 & 0.0421 \\
\hline \multicolumn{6}{|c|}{ Panel C: Benchmarks } \\
\hline LW1F & 0.0756 & 0.0631 & 0.0467 & 0.0379 & 0.0581 \\
\hline LWID & 0.0737 & 0.0614 & 0.0482 & 0.0394 & 0.0503 \\
\hline LWCC & 0.0712 & 0.0550 & 0.0496 & 0.0380 & 0.0540 \\
\hline LWNLS & 0.0763 & 0.0625 & 0.0465 & 0.0382 & 0.0545 \\
\hline KDM & 0.0606 & 0.0452 & 0.0498 & 0.0398 & 0.0428 \\
\hline
\end{tabular}


Table 10: Average monthly turnover in empirical data sets based on daily observations

This table reports the average monthly turnover of our averaging rule on the portfolio weight (AV-Wgt), the inverse (AV-Inv), and the covariance (AV-Cov) level in Panel A, of the single strategies in Panel B, and of the benchmarks in Panel C. The results are shown for to the Fama-French factor-mimicking and industry portfolios, as well as the STOCK500 data set. The out-of-sample period is from 06/1976 - 06/2019. The abbreviations for the strategies in Panels $\mathrm{B}$ and $\mathrm{C}$, as well as for the data sets, are reported in Tables 1 and 7 , respectively. Turnover is measured as the average percentage of total wealth traded in each period. The reported numbers are in percentages.

\begin{tabular}{lccccr}
\hline & $6 \mathrm{FF}$ & $25 \mathrm{FF}$ & 10Ind & 30Ind & STOCK500 \\
\hline Panel A: & Averaging rule & & & & \\
AV-Wgt & 11.35 & 16.52 & 4.72 & 8.51 & 11.36 \\
AV-Inv & 11.49 & 16.62 & 4.76 & 8.59 & 11.27 \\
AV-Cov & 11.32 & 16.31 & 4.79 & 8.56 & 13.03
\end{tabular}

Panel B: Single strategies

$\begin{array}{lrrrrr}\text { Sample } & 12.07 & 17.67 & 5.78 & 10.49 & 49.57 \\ \text { 1F } & 9.39 & 16.22 & 7.61 & 11.00 & 12.27 \\ \text { CC } & 7.40 & 15.21 & 6.29 & 9.76 & 16.49 \\ \text { ID } & 1.48 & 1.68 & 2.32 & 2.87 & 5.45 \\ \text { SC } & 0.46 & 0.94 & 1.10 & 1.86 & 3.65 \\ \text { POET } & 15.96 & 18.25 & 6.62 & 11.28 & 17.75 \\ & & & & & \\ \text { Panel C: Benchmarks } & & & 10.11 & 22.64 \\ \text { LW1F } & 11.70 & 17.24 & 5.74 & 9.63 & 33.38 \\ \text { LWID } & 9.05 & 15.58 & 5.00 & 9.58 & 26.65 \\ \text { LWCC } & 8.64 & 15.18 & 5.44 & 10.01 & 18.70 \\ \text { LWNLS } & 11.82 & 17.19 & 5.70 & 11.00 & 12.27 \\ \text { KDM } & 9.39 & 16.22 & 7.60 & & \end{array}$


Table 11: Average daily short interest in empirical data sets

This table reports the average daily short interest of our averaging rule on the portfolio weight (AV-Wgt), the inverse (AV-Inv), and the covariance (AV-Cov) level in Panel A, of the single strategies in Panel B, and of the benchmarks in Panel C. The results are shown for to the Fama-French factor-mimicking and industry portfolios, as well as the STOCK500 data set. The out-of-sample period is from 06/1976 - 06/2019. The abbreviations for the strategies in Panels B and $\mathrm{C}$, as well as for the data sets, are reported in Tables 1 and 7 respectively. Short interest is measured by the average amount of wealth that is held in short positions. The reported numbers are in percentages.

\begin{tabular}{lcrrrr}
\hline & $6 \mathrm{FF}$ & $25 \mathrm{FF}$ & 10Ind & 30 Ind & STOCK500 \\
\hline Panel A: & Averaging rule & & & & \\
FAWgt & 143.50 & 201.65 & 45.52 & 75.14 & 97.75 \\
FAInv & 145.02 & 202.89 & 45.87 & 76.00 & 96.67 \\
FACov & 143.57 & 197.73 & 45.78 & 75.51 & 115.48 \\
& & & & & \\
Panel B: Single & strategies & & & & \\
Sample & 151.70 & 215.21 & 57.83 & 97.21 & 366.94 \\
1F & 102.92 & 156.50 & 68.81 & 88.75 & 62.30 \\
CC & 87.44 & 146.64 & 55.29 & 74.97 & 85.18 \\
ID & 0.00 & 0.00 & 0.00 & 0.00 & 0.00 \\
SC & 0.00 & 0.00 & 0.00 & 0.00 & 0.00 \\
POET & 191.03 & 210.65 & 67.52 & 97.94 & 113.66 \\
\multicolumn{2}{l}{ Panel C: Benchmarks } & & & & \\
LW1F & 147.10 & 209.67 & 57.28 & 92.65 & 156.13 \\
LWID & 114.82 & 189.64 & 48.04 & 87.77 & 240.22 \\
LWCC & 107.85 & 180.44 & 52.12 & 88.00 & 192.04 \\
LWNLS & 149.13 & 209.27 & 56.74 & 91.52 & 113.18 \\
KDM & 102.92 & 156.50 & 68.81 & 88.75 & 62.30 \\
\hline
\end{tabular}


Figure 1: Average model weights in the simulated data sets

This figure plots the average weight over all 10,000 simulations that our averaging rule assigns to one of the considered estimators for estimation window sizes of $\tau=\{60,120,240,480,960\}$. Panel A shows the mean averaging weights when averaging is conducted on the portfolio weight level (AV-Wgt), Panel B when averaging is conducted on the the inverse (AV-Inv), and Panel C when averaging is conducted on the portfolio weight level (AV-Wgt). The abbreviations for the six single strategies are reported in Panel B of Table 1.
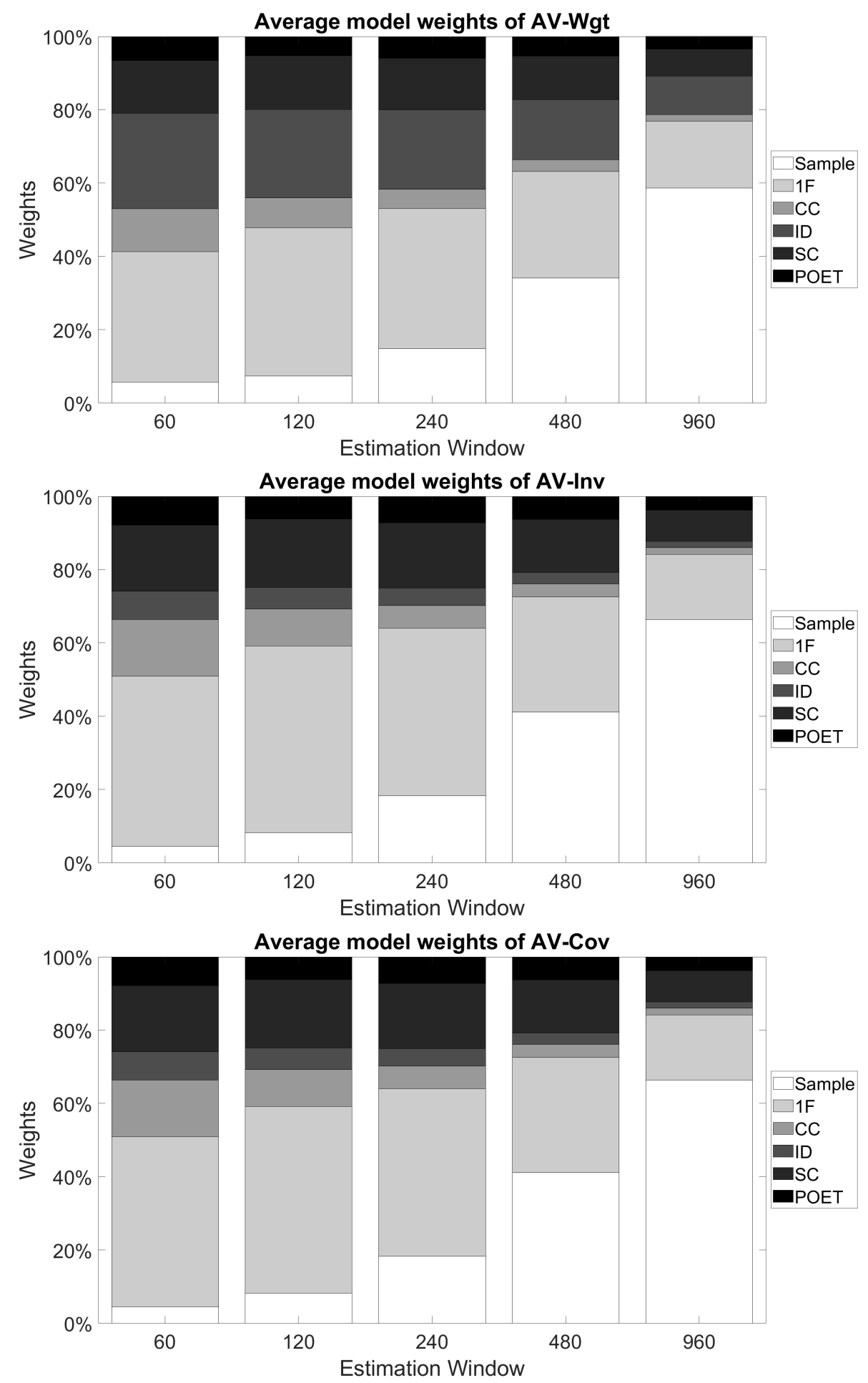
Figure 2: Average strategy weights of AV-Wgt for portfolios and stocks

This figure plots the averaging weights of the averaging rule on the portfolio weight level (AVWgt) over the out-of-sample period from 06/1976 - 06/2019. The weights represent the allocation of the respective estimator within the averaging rule over the holding period, which comprises 21 trading days. Panel A shows the plot for the $6 \mathrm{FF}$, Panel B for the 25FF, Panel C for the 10Ind, Panel D for the 30Ind, and Panel E for the Stock500 data set. The abbreviations for the portfolio strategies and data sets are reported in Tables 1 and 7 , respectively.
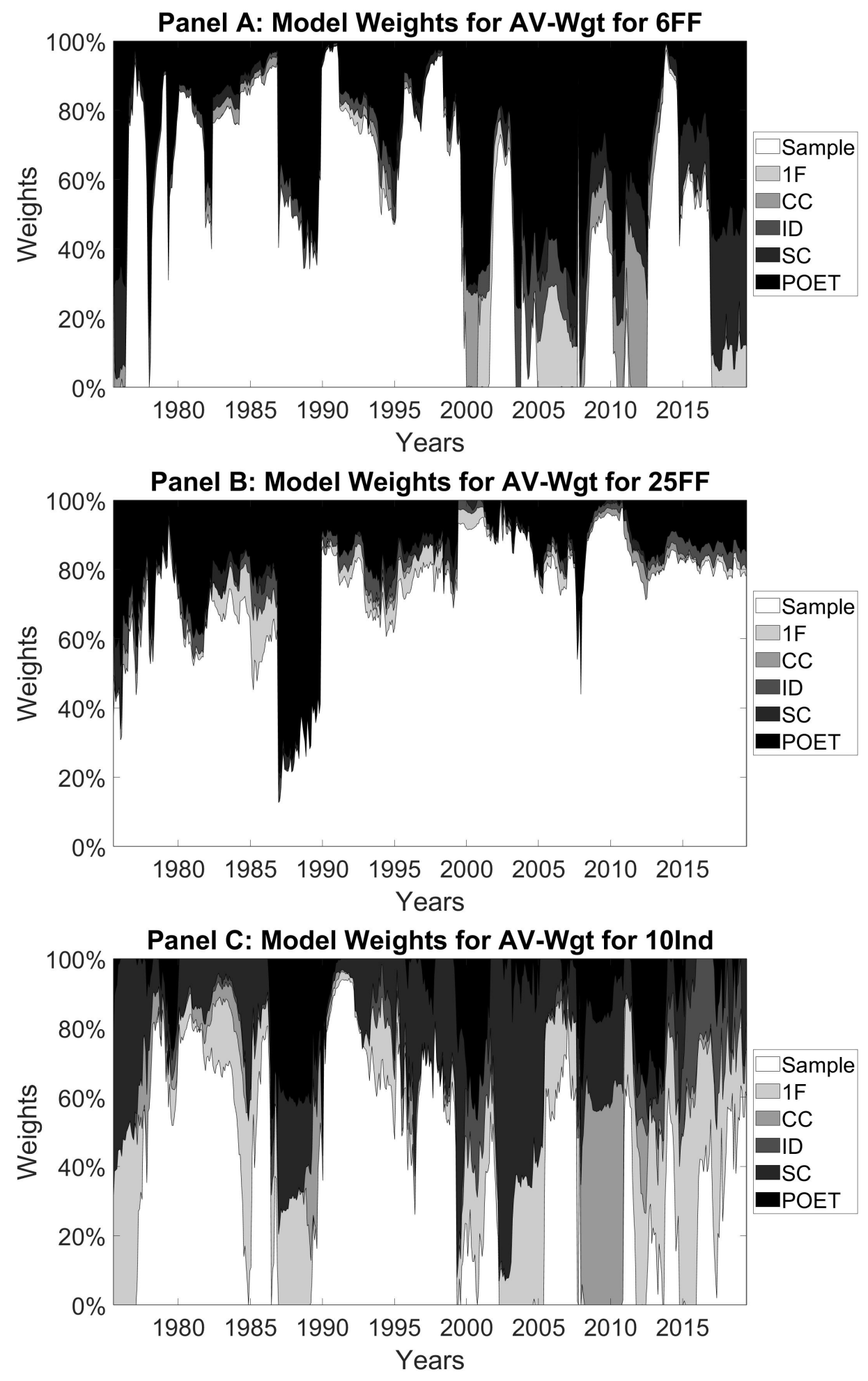

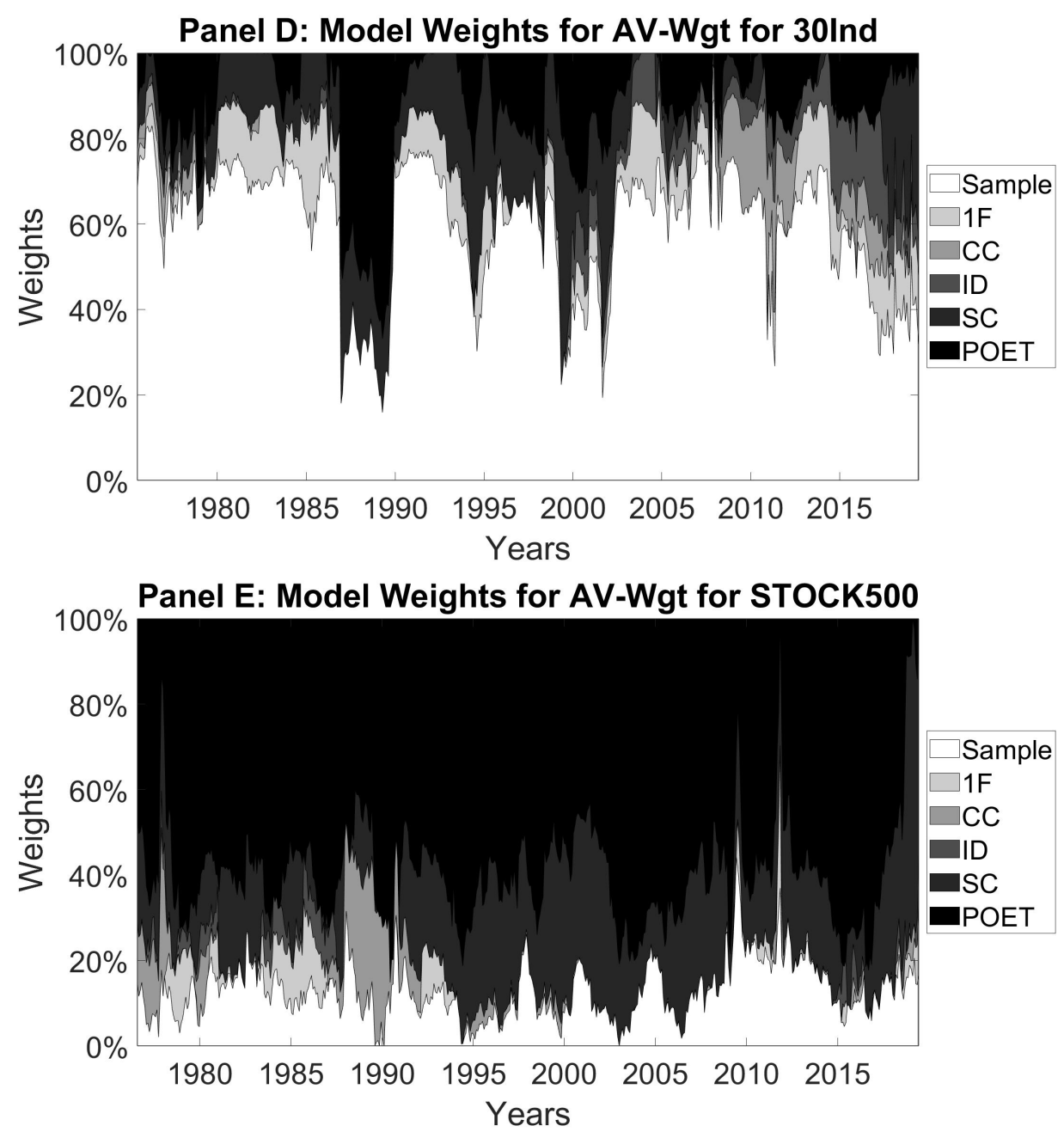


\title{
Internet Appendix for \\ Diversifying estimation errors: \\ An efficient averaging rule for portfolio optimization
}

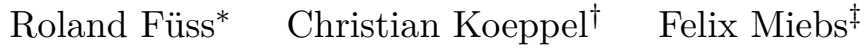

This Version: February 8, 2021

\begin{abstract}
We propose an averaging rule that combines established minimum-variance strategies to minimize the expected out-of-sample variance. Our rule overcomes the problem of selecting the "best" strategy ex-ante and diversifies remaining estimation errors of the single strategies included in the averaging. Extensive simulations show that the contributions of estimation errors to the out-of-sample variances are uncorrelated between the considered strategies. This implies that averaging over multiple strategies offers sizable diversification benefits. Our rule leverages these benefits and compares favorably to eleven strategies in terms of out-of-sample variance on both simulated and empirical data sets. The Sharpe ratio is across all data sets at least $25 \%$ higher than for the $1 / \mathrm{N}$ portfolio.
\end{abstract}

\section{JEL Classification: G11}

Keywords: Averaging; diversification; estimation error; portfolio optimization; shrinkage.

\footnotetext{
*Swiss Institute of Banking and Finance (s/bf), University of St.Gallen, Unterer Graben 21, 9000 St. Gallen, Switzerland, Research Associate at the Centre for European Economic Research (ZEW), Mannheim, Germany and a Visiting Professor and Fellow at the Center for Real Estate and Environmental Economics at NTNU Business School, Trondheim, Norway; Phone: +41 (0)71 224-7055, Fax: +41 (0)71 224-7088; Email: roland.fuess@unisg.ch ${ }^{\dagger}$ University of St.Gallen, Unterer Graben 21, 9000 St. Gallen, Switzerland; Phone: +41 (0)76 38 30 757, Email: christian.koeppel@live.com

${ }^{\ddagger}$ University of Applied Sciences Cologne, Claudiusstr. 1, 50678 Cologne, Germany; Phone: +49 (0)221 82753792 ; Email: felix.miebs@th-koeln.de.
} 


\section{A Internet Appendix}

In this appendix, we present supplementary charts and additional tests with the following structure:

Appendix A.1: Description of estimators

Appendix A.2: Additional simulation results

Appendix A.3: Empirical study on monthly excess returns 


\section{A.1 Description of estimators}

In this section, we describe the considered covariance matrix estimators introduced in section 2.2 .

\section{A.1.1 Sample estimator}

The Sample estimator is denoted as $\hat{\Sigma}_{S}$ and is based on the unbiased estimation of the vector of means of the in-sample asset excess returns in the estimation window, i.e. the sample size $\tau$ :

$$
\hat{\Sigma}_{S}=(1 /(\tau-1)) \Sigma_{t=1}^{\tau}\left(r_{i, t}-\mu_{S}\right)\left(r_{i, t}-\mu_{S}\right)^{\prime},
$$

where $\mu_{S}=(1 / \tau) \sum_{t=1}^{\tau} r_{i, t}$ is the unbiased sample vector of means and $r_{i, t}$ is the excess return of stock $i$ at time $t$. Similar to existing shrinkage strategies, our averaging rule combines the Sample estimator with one or more of the following single strategies.

\section{A.1.2 Single-factor model}

The return of stock $i$ at time $t, r_{i, t}$, is in the single-factor model of Sharpe (1967) described as:

$$
r_{i, t}=\alpha_{i}+\beta_{i} r_{M K T, t}+\epsilon_{i, t},
$$

where $\alpha_{i}$ is the mispricing of stock $i, \beta_{i}$ is the beta factor of stock $i, r_{M K T, t}$ is the excess return of the market over the risk-free asset at time $t$, and $\epsilon_{i, t}$ is the residual return of stock $i$ at time $t$. Following Chan et al. (1999), the covariance matrix estimator $\hat{\Sigma}_{1 F}$ of the single-factor model $(1 \mathrm{~F})$ is given by:

$$
\hat{\Sigma}_{1 F}=\hat{\Sigma}_{M K T}^{2} \hat{\beta} \hat{\beta}^{\prime}+\hat{\Delta}
$$

where $\hat{\Sigma}_{M K T}^{2}, \hat{\beta}$, and $\hat{\Delta}$ are estimates of market variance, asset betas, and a diagonal matrix with the residual variances along the main diagonal, respectively.

\section{A.1.3 Constant correlation model}

Elton and Gruber 1973) suggest a structured estimator of the covariance matrix by assuming that each pair of assets has the identical correlation coefficient, while each of the $N$ assets has an individual standard deviation. The resulting constant correlation covariance matrix estimator is given by:

$$
\hat{\Sigma}_{C C}=\Lambda C \Lambda^{\prime}
$$

where $\Lambda$ denotes a diagonal matrix containing the standard deviations $\hat{\sigma}_{i, i}$ of the assets $i, j$, and $C$ is the constant correlation matrix with ones along the main diagonal and the average of all pairwise correlations $\hat{\sigma}_{i, j}$ off the main diagonal. 


\section{A.1.4 Multiple of the identity matrix}

This estimator implies that all assets are uncorrelated and exhibit equal variances. Following Ledoit and Wolf (2004a), the elements along the main diagonal are set to the average variance of all assets in the investment universe. The covariance matrix estimator for the scaled identity matrix model ID is given by:

$$
\hat{\Sigma}_{I D}=I \mu_{\hat{s}}, \text { with } \mu_{\hat{s}}=\frac{1}{N} \sum_{i=1}^{N} \hat{s}_{i}^{2},
$$

where $\hat{s}_{i}^{2}$ is the sample variance of asset $i$. The resulting minimum-variance portfolio corresponds to the $1 / \mathrm{N}$ portfolio.

\section{A.1.5 Principal components}

Fan et al. (2013) introduce the principal orthogonal complement thresholding method, POET, which uses an approximate factor model to define the risk factors based on principal components of the sample covariance matrix and impose a threshold on the remaining principal components after taking out the first $K$. The covariance matrix estimator $\hat{\Sigma}_{P O E T}$ of the POET model is given by:

$$
\hat{\Sigma}_{P O E T}=\sum_{i=1}^{K} \hat{\gamma}_{\tau, i} \hat{\xi}_{i} \hat{\xi}_{i}^{\prime}+\hat{R}_{K}^{T},
$$

where $\hat{\gamma}_{\tau, 1} \geq \hat{\gamma}_{\tau, 2} \geq \cdots \geq \hat{\gamma}_{\tau, p}$ are the ordered eigenvalues of the sample covariance matrix $\hat{\Sigma_{S}}$ over the estimation window $\tau,\left(\hat{\xi}_{i}\right)_{i=1}^{p}$ are the corresponding eigenvectors, $\hat{R}_{K}^{T}$ is the principal orthogonal complement of $K$ diverging eigenvalues of $\hat{\Sigma}_{S}$.

\section{A.1.6 Linear shrinkage estimators of the covariance matrix}

The linear shrinkage estimators of Ledoit and Wolf $(2003,2004 \mathrm{a} b)$ have the following general form:

$$
\hat{\Sigma}_{L W}=\phi \hat{F}+(1-\phi) \hat{\Sigma}_{S}
$$

The shrunken covariance matrix $\hat{\Sigma}_{L W}$ is a convex combination of the estimated sample covariance matrix $\hat{\Sigma}_{S}$ and a shrinkage target $\hat{F}$, with the shrinkage intensity, $\phi$, taking values between zero and one. The optimal shrinkage intensity is determined by minimizing the mean-squared error of $\hat{\Sigma}_{L W}$. The authors consider three different candidates for $\hat{F}$ : the single-factor model covariance matrix $\hat{\Sigma}_{L W 1 F}$ by Ledoit and Wolf (2003), a multiple of the identity matrix $\hat{\Sigma}_{L W I D}$ as in Ledoit and Wolf (2004a), and the constant correlation model implied covariance matrix $\hat{\Sigma}_{L W C C}$ by Ledoit and Wolf (2004b). 


\section{A.1.7 Shrinkage estimators for the inverse covariance matrix}

Kourtis et al. (2012) propose a shrinkage estimator of the inverse covariance matrix, which takes the general form for shrinking the inverse covariance matrix $\hat{\Sigma}^{\prime}$ as given by:

$$
\hat{\Sigma}^{-1}=\phi \hat{F}^{-1}+(1-\phi) \hat{\Sigma}_{S}^{-1}
$$

where $\hat{\Sigma}^{-1}$ is a convex combination of the estimated inverse sample covariance matrix $\hat{\Sigma}_{S}^{-1}$ and an inverse shrinkage target $\hat{F}^{-1}$, with the shrinkage intensity $\phi$.

Kourtis et al. (2012) use three particular targets: the identity, the inverse covariance matrix generated by the single-factor model of Sharpe (1963), and a weighted sum of these two matrices. The resulting strategies offer significant improvements in out-of-sample portfolio performance in terms of both variance and risk-adjusted excess returns compared to the GMVP, the $1 / \mathrm{N}$ portfolio, and benchmarks based on shrinkage estimators of the covariance matrix. This can be explained by the more immediate impact on portfolio weights by the inverse of the covariance matrix. They also highlight that more than one target further reduces estimation errors. We denote the three-fund strategy of Kourtis et al. (2012) as KDM.

\section{A.1.8 Non-linear shrinkage estimators for the inverse covariance matrix}

Ledoit and Wolf (2017) show that a non-linear shrinkage estimator (NLS) based on Stein (1975, 1986) outperforms linear shrinkage both on the covariance matrix and its inverse by correcting the over-dispersed eigenvalues of the sample covariance matrix. This approach is asymptotically optimal for portfolio selection when the number of assets $N$ is of the same magnitude as the sample size $\tau$. The non-linear shrinkage estimator for the NLS model is given by:

$$
\begin{aligned}
& \hat{\Sigma}_{N L S}:= U_{\tau} \hat{D}_{\tau} U_{\tau}^{\prime} \text { where } \hat{D}_{\tau}:=\operatorname{Diag}\left(\hat{d}_{\tau}\left(\gamma_{\tau, 1}\right), \ldots, \hat{d}_{\tau}\left(\gamma_{\tau, N}\right)\right) \text { and } \\
& \hat{d}_{\tau}\left(\gamma_{\tau, i}\right):=\left\{\begin{array}{l}
\frac{1}{\gamma_{\tau, i}\left|\hat{c}\left(\gamma_{\tau, i}\right)\right|^{2}} \text { if } \gamma_{\tau, i}>0, \\
\frac{1}{\left(\frac{N}{\tau}-1\right) \hat{c}(0)} \text { if } N>\tau \text { and } \gamma_{\tau, i}=0,
\end{array}\right.
\end{aligned}
$$

where $U$ is an orthogonal matrix from a spectral decomposition of the sample covariance matrix $\hat{\Sigma}_{S}, \hat{c}$ is the complex-valued Stieltjes transformation, and $\gamma_{\tau, i}$ are the sample eigenvalues, sorted in increasing order.

\section{A.1.9 Short-sale constraints}

Jagannathan and $\mathrm{Ma}$ (2003) provide a moment shrinkage interpretation of short-sale constraints in the minimum-variance portfolio optimization problem. The additional weight constraint is formulated as:

$$
\hat{w}_{i} \geq 0, \text { for } i=1,2, \ldots, N \text {. }
$$


Jagannathan and Ma 2003) show that the asset weights of the short-sale constrained minimumvariance portfolio correspond to those of the GMVP based on the following covariance estimator:

$$
\hat{\Sigma}_{S C}=\hat{\Sigma}_{S}-\left(\delta 1^{\prime}+1 \delta^{\prime}\right)
$$

where $\delta=\left(\delta_{1}, \delta_{2}, \ldots, \delta_{N}\right)$ is the vector of Lagrange multipliers for the non-negativity constraints in Equation 27. 


\section{A.2 Additional simulation results}

This section contains the supporting figures for our simulation study. Complementing the results for the out-of-sample variance in Table 4, we report the results for the average out-of-sample Sharpe ratio in Table A.1. Similar to the variance, we find that the average Sharpe ratio increases with the number of strategies.

In the left column of Figures A.1, A.2, and A.3, we evaluate the distribution of the out-of-sample variances for AV-Wgt, AV-Inv, and AV-Cov with the kernel densities over all simulation runs and permutations. We observe that the distributions for $M=6$ center around smaller out-of-sample variances and collapse more tightly around this value than the distributions for smaller values of $M$. The result holds for all averaging levels and supports the finding that combining all six strategies is beneficial when compared to averaging over a subset. Investigating the distribution of the out-of-sample Sharpe ratios for AV-Wgt, AV-Inv, and AV-Cov, we turn to the right column of Figures A.1, A.2, and A.3. We find that the distributions for $M=6$ are centered around a higher mean value and collapse more tightly around the mean value for estimation windows up to $\tau=120$. We observe that this effect diminishes as the estimation window increases.

In Figures A.4 A.5, and A.6, we investigate the out-of-sample variances of our averaging rule in relation to the existing strategies in more detail, exploring the kernel densities for AV-Wgt, AV-Inv, and AV-Cov, respectively. The left column in each figure shows the densities in comparison to the single strategies. We find that the distributions of AV-Wgt, AV-Inv, and AV-Cov are centered around a smaller mean value and collapse more tightly for estimation windows up to $\tau=480$ in comparison to the aforementioned strategies. The kernel densities of each averaging level of our rule also compare favorably to the benchmarks. We find that the distributions of AV-Wgt, AV-Inv, and AV-Cov collapse as tightly as the competing strategies, but around a smaller mean value. This observation holds for estimation windows of up to $\tau=480$.

The kernel density plots of the out-of-sample Sharpe ratios for AV-Wgt, AV-Inv, and AV-Cov in Figures A.7, A.8, and A.9 corroborate the favorable comparison relative to the existing strategies. The left column of the aforementioned figures presents the results in comparison to the single strategies. We observe that the distribution of the out-of-sample Sharpe ratios for all averaging levels of our rule collapses more tightly around a higher mean value than the aforementioned strategies, except for SC. The right column of the aforementioned figures shows the results in comparison to the benchmarks. We find that our rule centers around a higher mean value for all estimation windows and collapses more tightly around the respective mean for estimation windows up to $\tau=120$. 
Table A.1: Sharpe ratio comparison of averaging strategies in terms of averaged strategies

This table reports the average out-of-sample Sharpe ratios of our proposed averaging rule on the portfolio weight (AV-Wgt), the inverse (AV-Inv), and the covariance matrix (AV-Cov) level over 10,000 simulation runs for a varying number of single strategies, $M$. The out-of-sample Sharpe ratio in the $k$-th simulation run is computed as $\hat{\Psi}_{k}=\left(\hat{w}_{k, m}^{\prime} \mu_{k}\right)\left(\hat{w}_{k, m} \Sigma_{k} \hat{w}_{k, m}\right)^{-1 / 2}$, where $\mu_{k}$ is the population mean vector, and $\Sigma_{k}$ is the population covariance matrix resulting from the random pairing of the assets' factor loadings, their mispricing, as well as their idiosyncratic volatility. $\hat{w}_{k, m}$ are the estimated portfolio weights of the $m$-th strategy based on the $\tau$ simulated returns. Panels A - E show the respective results for estimation windows of $\tau=\{60,120,240,480,960\}$. The results for $M=2$ up to $M=6$ represent averages over all possible permutations from combining the Sample with the remaining five strategies from Panel B in Table 1 . The reported average out-of-sample Sharpe ratios are scaled by $10 \mathrm{e}+03$.

\begin{tabular}{|c|c|c|c|c|c|}
\hline & $M=2$ & $M=3$ & $M=4$ & $M=5$ & $M=6$ \\
\hline \multicolumn{6}{|c|}{ Panel A: Estimation window $\tau=60$} \\
\hline AV-Wgt & 132.293 & 136.889 & 138.788 & 140.256 & 140.320 \\
\hline AV-Inv & 132.421 & 136.980 & 138.817 & 140.278 & 140.341 \\
\hline $\mathrm{AV}-\mathrm{Cov}$ & 133.496 & 136.412 & 138.077 & 138.896 & 139.006 \\
\hline \multicolumn{6}{|c|}{ Panel B: Estimation window $\tau=120$} \\
\hline AV-Wgt & 135.646 & 139.653 & 141.141 & 142.527 & 142.616 \\
\hline AV-Inv & 135.625 & 139.636 & 141.107 & 142.480 & 142.571 \\
\hline $\mathrm{AV}-\mathrm{Cov}$ & 136.772 & 139.582 & 140.992 & 141.674 & 141.766 \\
\hline \multicolumn{6}{|c|}{ Panel C: Estimation window $\tau=\mathbf{2 4 0}$} \\
\hline AV-Wgt & 138.562 & 141.202 & 142.315 & 143.496 & 143.620 \\
\hline AV-Inv & 138.542 & 141.176 & 142.283 & 143.452 & 143.579 \\
\hline $\mathrm{AV}-\mathrm{Cov}$ & 139.199 & 141.309 & 142.414 & 142.960 & 143.051 \\
\hline \multicolumn{6}{|c|}{ Panel D: Estimation window $\tau=480$} \\
\hline AV-Wgt & 140.270 & 141.819 & 142.535 & 143.435 & 143.577 \\
\hline AV-Inv & 140.277 & 141.795 & 142.510 & 143.399 & 143.547 \\
\hline $\mathrm{AV}-\mathrm{Cov}$ & 140.769 & 142.133 & 142.939 & 143.319 & 143.410 \\
\hline \multicolumn{6}{|c|}{ Panel E: Estimation window $\tau=960$} \\
\hline AV-Wgt & 141.527 & 142.359 & 142.764 & 143.373 & 143.478 \\
\hline AV-Inv & 141.541 & 142.346 & 142.747 & 143.348 & 143.460 \\
\hline $\mathrm{AV}-\mathrm{Cov}$ & 141.639 & 142.413 & 142.915 & 143.161 & 143.230 \\
\hline
\end{tabular}


Figure A.1: Kernel densities of the out-of-sample variances and Sharpe ratios of AV-Wgt

This figure plots the kernel densities of the out-of-sample variances and Sharpe ratios of our averaging rule on the portfolio weight (AV-Wgt) level over 10,000 simulation runs for a varying number of single strategies, $M$. The densities for $M=2$ up to $M=6$ are based on all possible permutations from combining the Sample with the remaining five strategies from Panel B in Table1. The out-of-sample variance in the $k$-th simulation run is computed as $\hat{\sigma}_{k}^{2}=\hat{w}_{k, m}^{\prime} \Sigma_{k} \hat{w}_{k, m}$, where $\Sigma_{k}$ is the population covariance matrix resulting from the random pairing of the assets' factor loadings, their mispricing, as well as their idiosyncratic volatility. $\hat{w}_{k, m}$ are the estimated portfolio weights of the $m$-th strategy based on the $\tau$ simulated returns. The out-of-sample Sharpe ratio in the $k$-th simulation run is computed as $\hat{\Psi}_{k}=\left(\hat{w}_{k, m}^{\prime} \mu_{k}\right)\left(\hat{w}_{k, m} \Sigma_{k} \hat{w}_{k, m}\right)^{-1 / 2}$, where $\mu_{k}$ is the population mean vector. Panels A - E show the respective results for estimation windows of $\tau=\{60,120,240,480,960\}$. The reported variances and Sharpe ratios are scaled by $10 \mathrm{e}+03$.
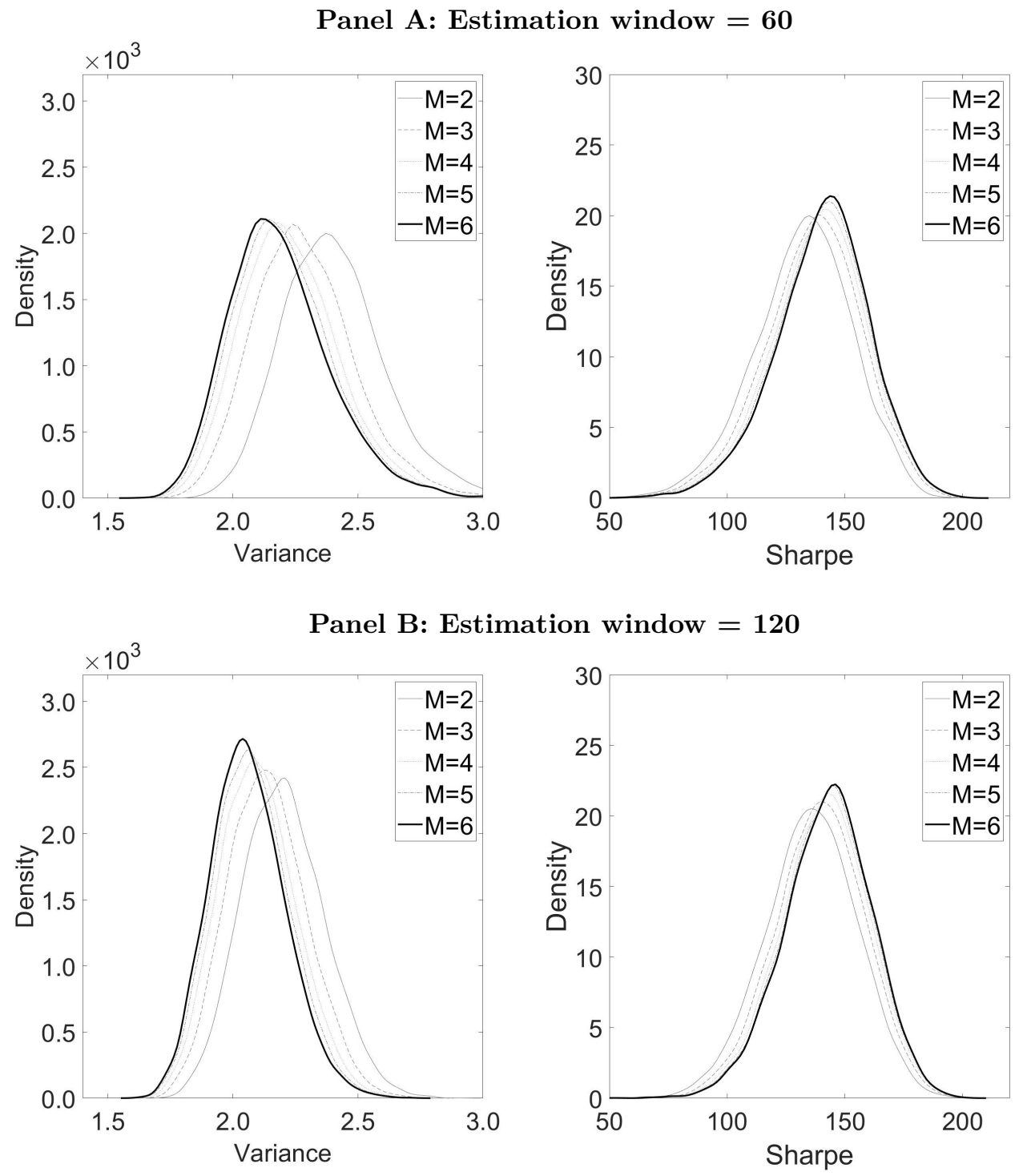

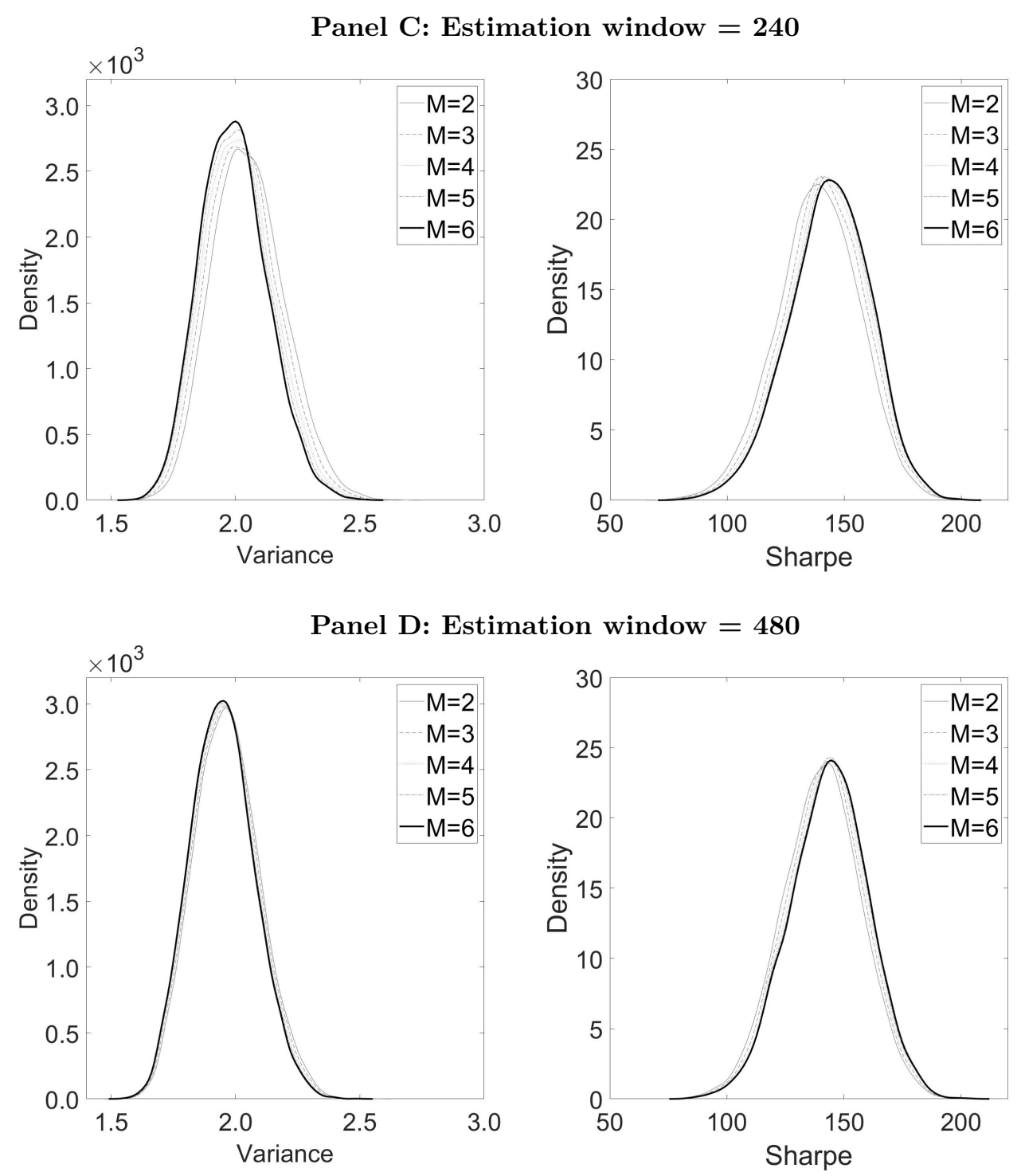

Panel E: Estimation window $=960$

Figure A.2: Kernel densities of the out-of-sample variances and Sharpe ratios of AV-Inv

This figure plots the kernel densities of the out-of-sample variances and Sharpe ratios of our averaging rule on the inverse covariance matrix (AV-Inv) level over 10,000 simulation runs for a varying number of single strategies, $M$. The densities for $M=2$ up to $M=6$ are based on all possible permutations from combining the Sample with the remaining five strategies from Panel B in Table 1. The out-of-sample variance in the $k$-th simulation run is computed as $\hat{\sigma}_{k}^{2}=\hat{w}_{k, m}^{\prime} \Sigma_{k} \hat{w}_{k, m}$, where $\Sigma_{k}$ is the population covariance matrix resulting from the random pairing of the assets' factor loadings, their mispricing, as well as their idiosyncratic volatility. $\quad \hat{w}_{k, m}$ are the estimated portfolio weights of the $m$-th strategy based on the $\tau$ simulated returns. The out-of-sample Sharpe ratio in the $k$-th simulation run is computed as $\hat{\Psi}_{k}=\left(\hat{w}_{k, m}^{\prime} \mu_{k}\right)\left(\hat{w}_{k, m} \Sigma_{k} \hat{w}_{k, m}\right)^{-1 / 2}$, where $\mu_{k}$ is the population mean vector. Panels A - E show the respective results for estimation windows of $\tau=\{60,120,240,480,960\}$. The reported variances and Sharpe ratios are scaled by $10 \mathrm{e}+03$.
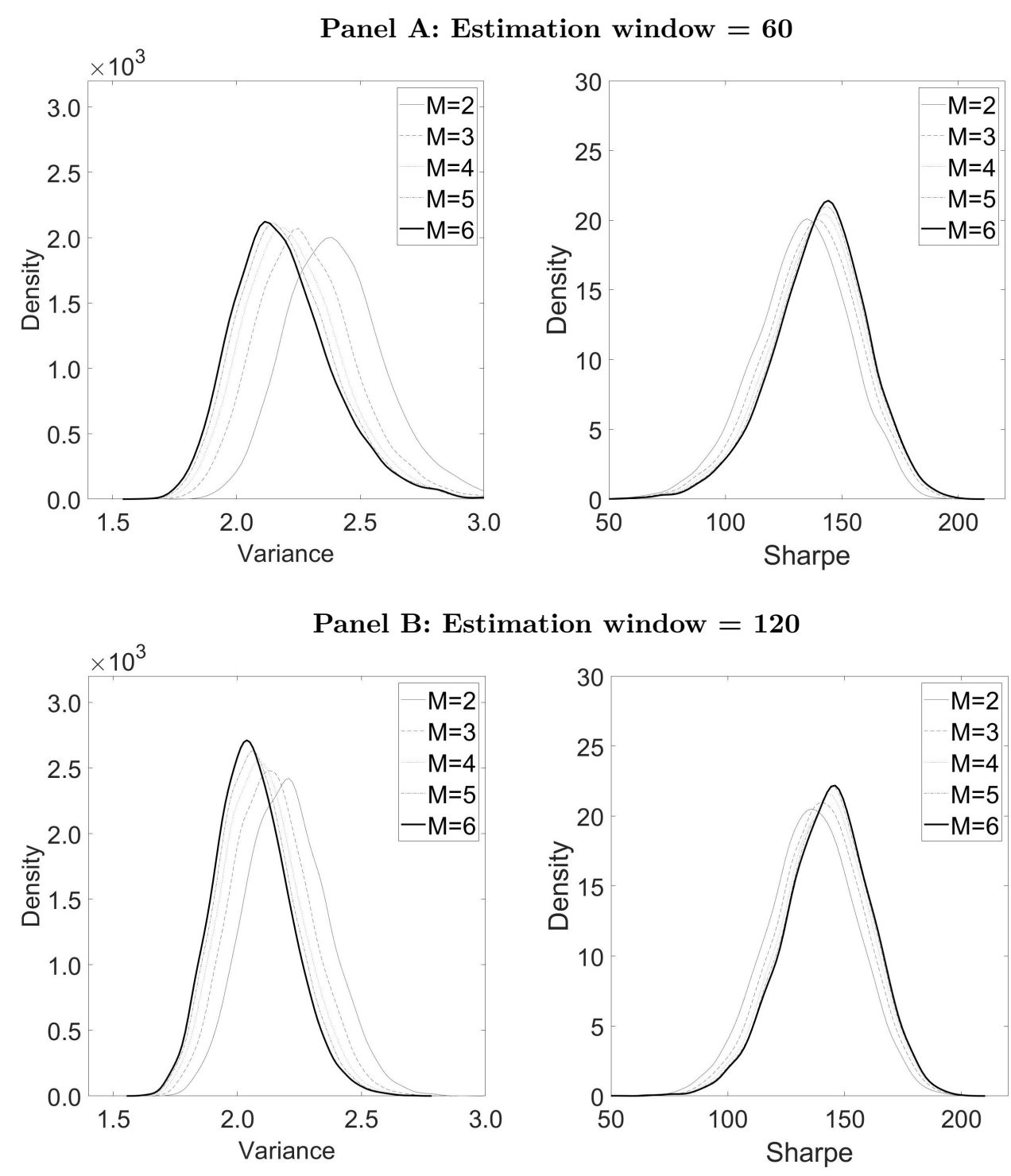

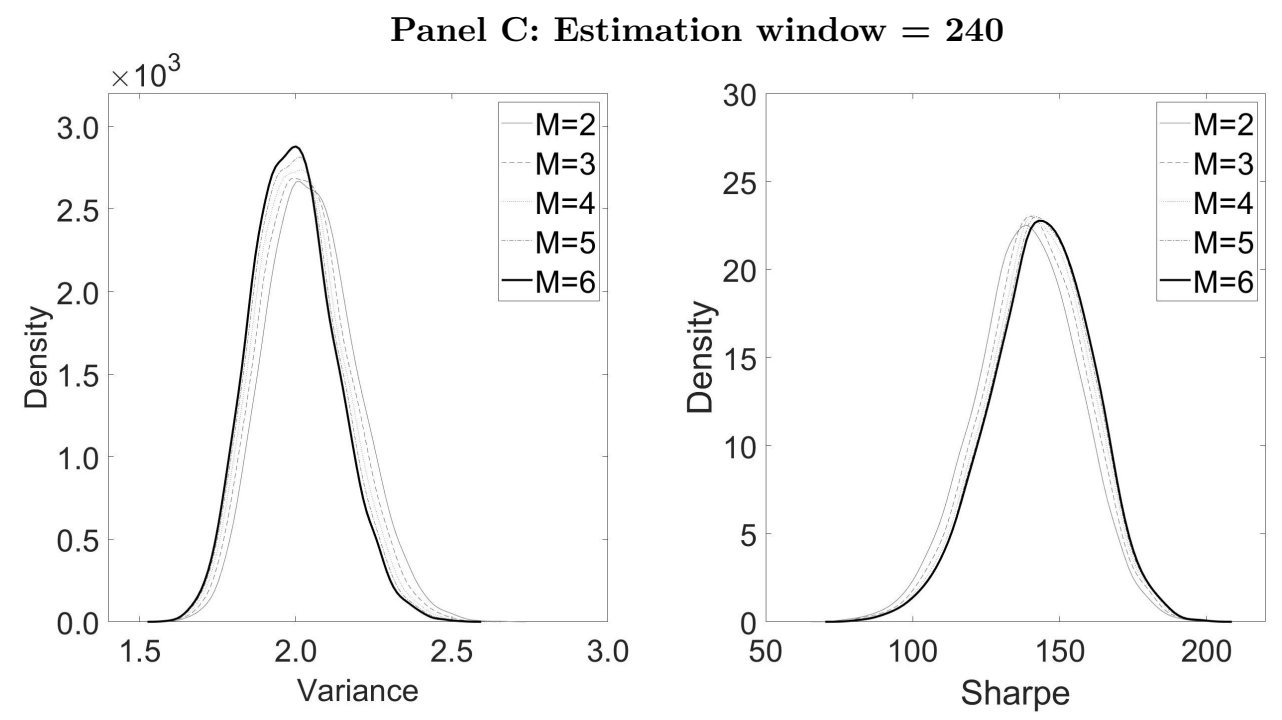

Panel D: Estimation window $=480$


Panel E: Estimation window $=960$
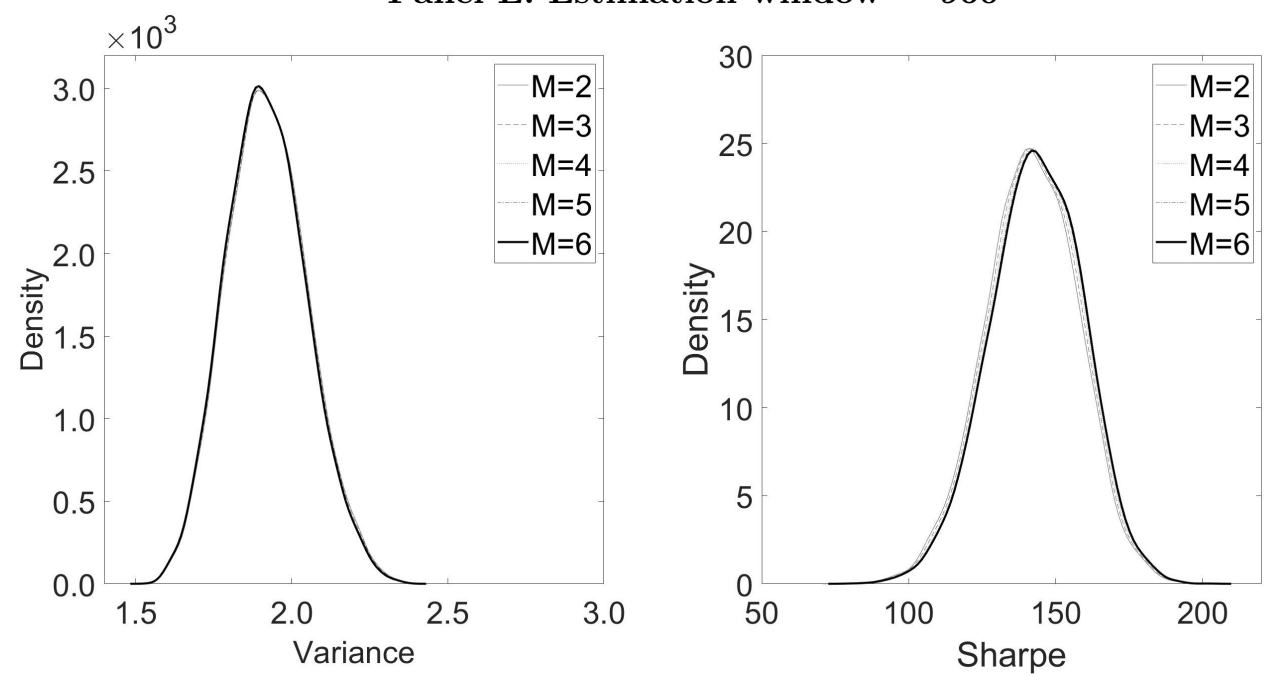
Figure A.3: Kernel densities of the out-of-sample variances and Sharpe ratios of AV-Cov

This figure plots the kernel densities of the out-of-sample variance and Sharpe ratio of our averaging rule on the covariance matrix (AV-Cov) level over 10,000 simulation runs for a varying number of single strategies, $M$. The densities for $M=2$ up to $M=6$ are based on all possible permutations from combining the Sample with the remaining five strategies from Panel B in Table1. The out-of-sample variance in the $k$-th simulation run is computed as $\hat{\sigma}_{k}^{2}=\hat{w}_{k, m}^{\prime} \Sigma_{k} \hat{w}_{k, m}$, where $\Sigma_{k}$ is the population covariance matrix resulting from the random pairing of the assets' factor loadings, their mispricing, as well as their idiosyncratic volatility. $\hat{w}_{k, m}$ are the estimated portfolio weights of the $m$-th strategy based on the $\tau$ simulated returns. The out-of-sample Sharpe ratio in the $k$-th simulation run is computed as $\hat{\Psi}_{k}=\left(\hat{w}_{k, m}^{\prime} \mu_{k}\right)\left(\hat{w}_{k, m} \Sigma_{k} \hat{w}_{k, m}\right)^{-1 / 2}$, where $\mu_{k}$ is the population mean vector. Panels A - E show the respective results for estimation windows of $\tau=\{60,120,240,480,960\}$. The reported variances and Sharpe ratios are scaled by $10 \mathrm{e}+03$.
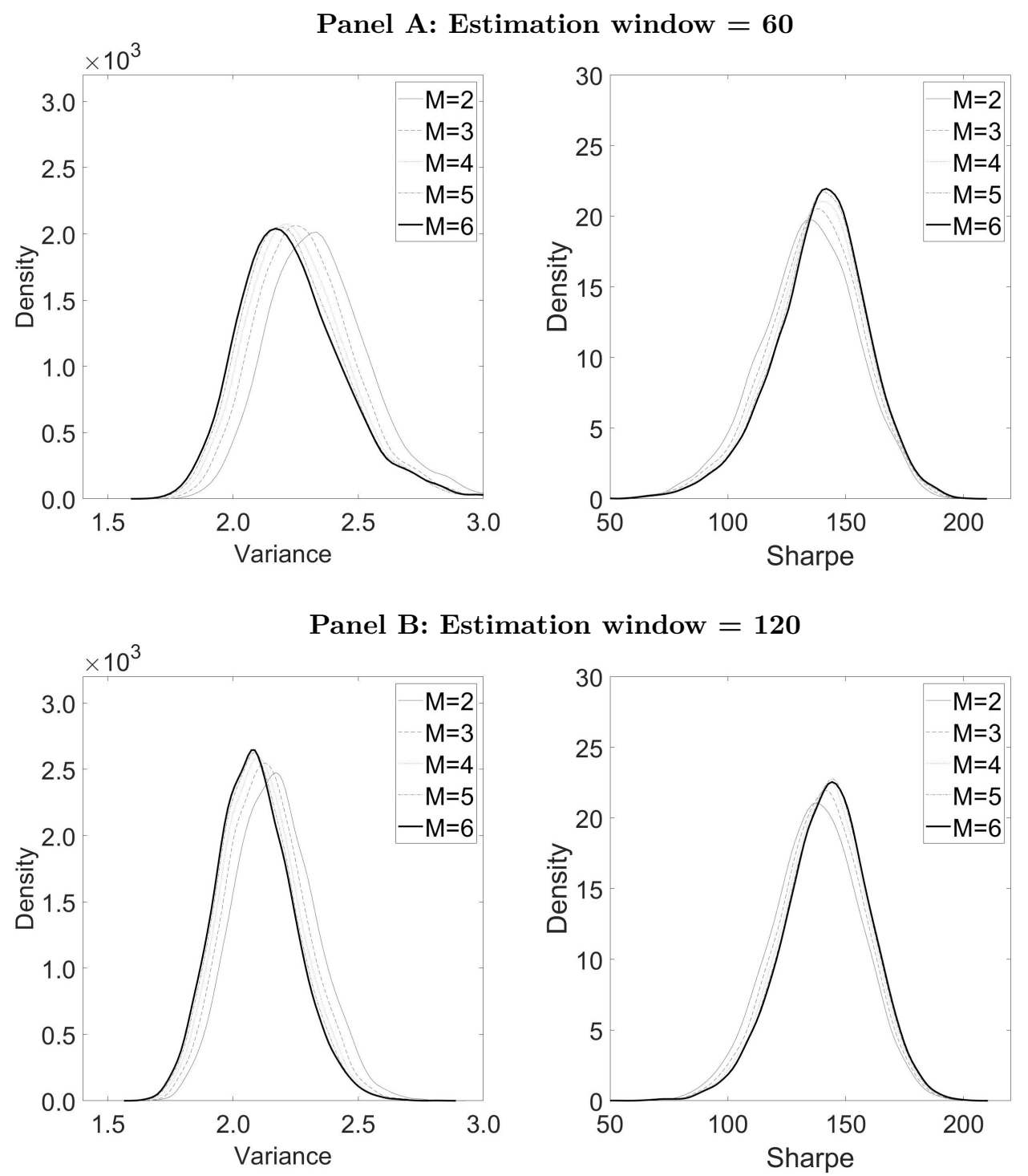

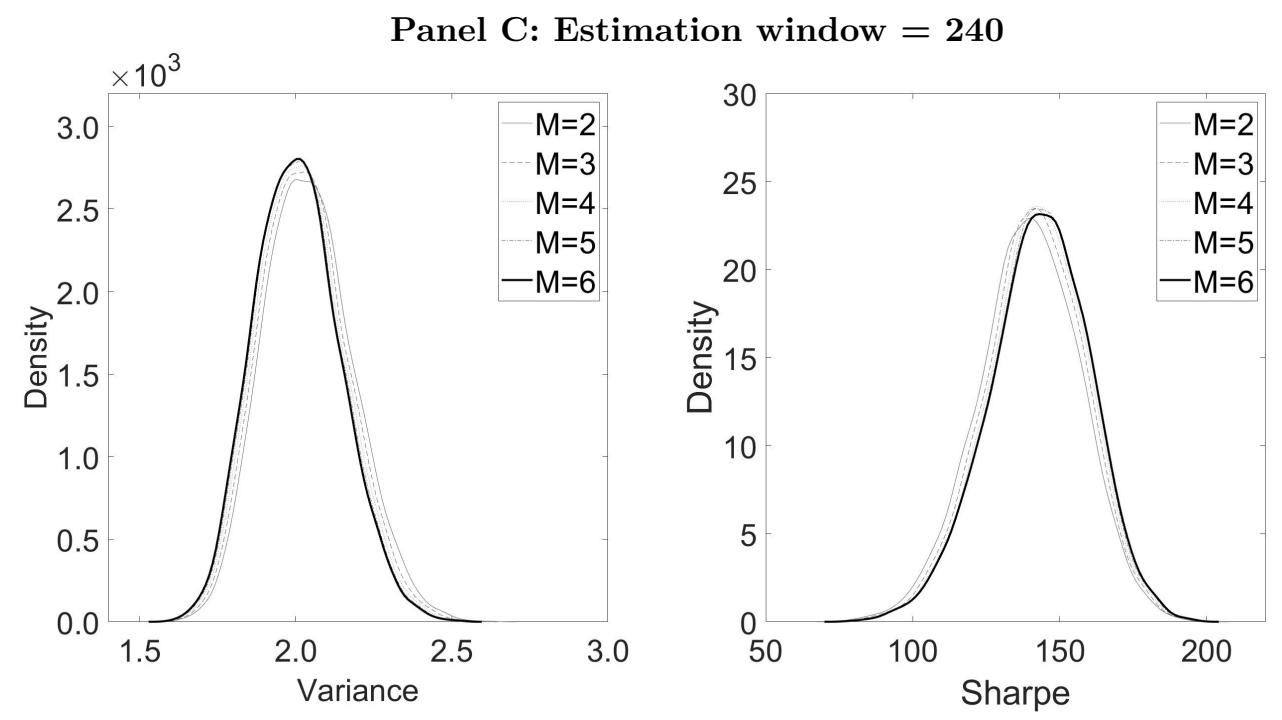

Panel D: Estimation window $=480$
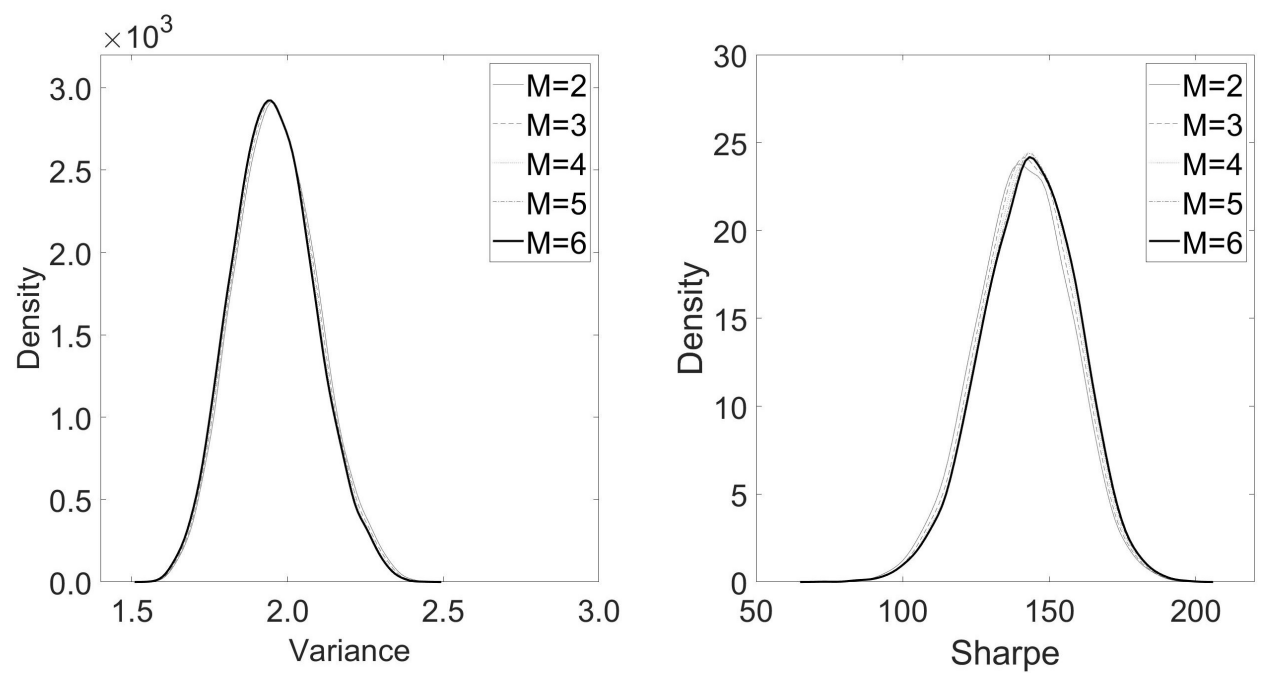

Panel E: Estimation window $=960$
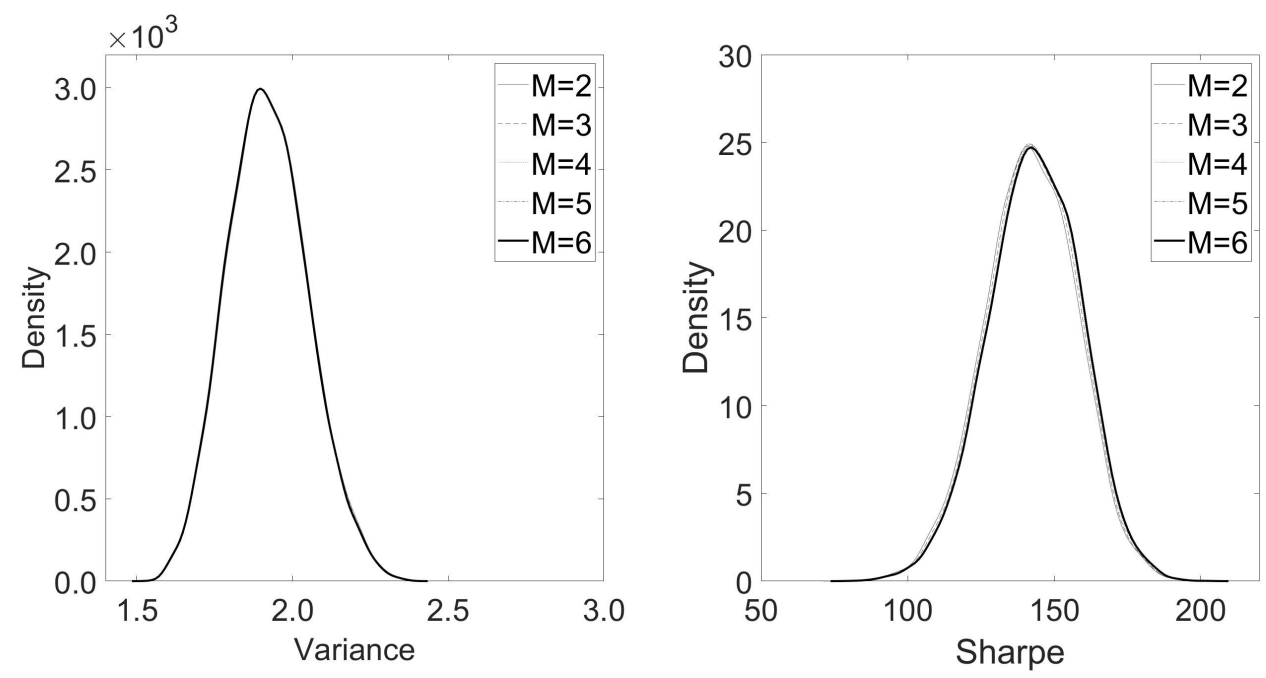
Figure A.4: Kernel densities of the out-of-sample variances of AV-Wgt compared to established strategies and benchmarks

This figure plots the kernel densities of the out-of-sample variances of our averaging rule on the portfolio weight (AV-Wgt) level over 10,000 simulation runs in comparison to the single strategies (left column) and the benchmarks (right column) for the considered estimation windows of $\tau=\{60,120,240,480,960\}$. The out-of-sample variances are scaled by $10 \mathrm{e}+03$. The results for AV-Wgt are based on averaging over the $M=6$ strategies from Panel B in Table 1. For illustration purpose only, we exclude the ID strategy because it distorts the visualization of the remaining strategies.
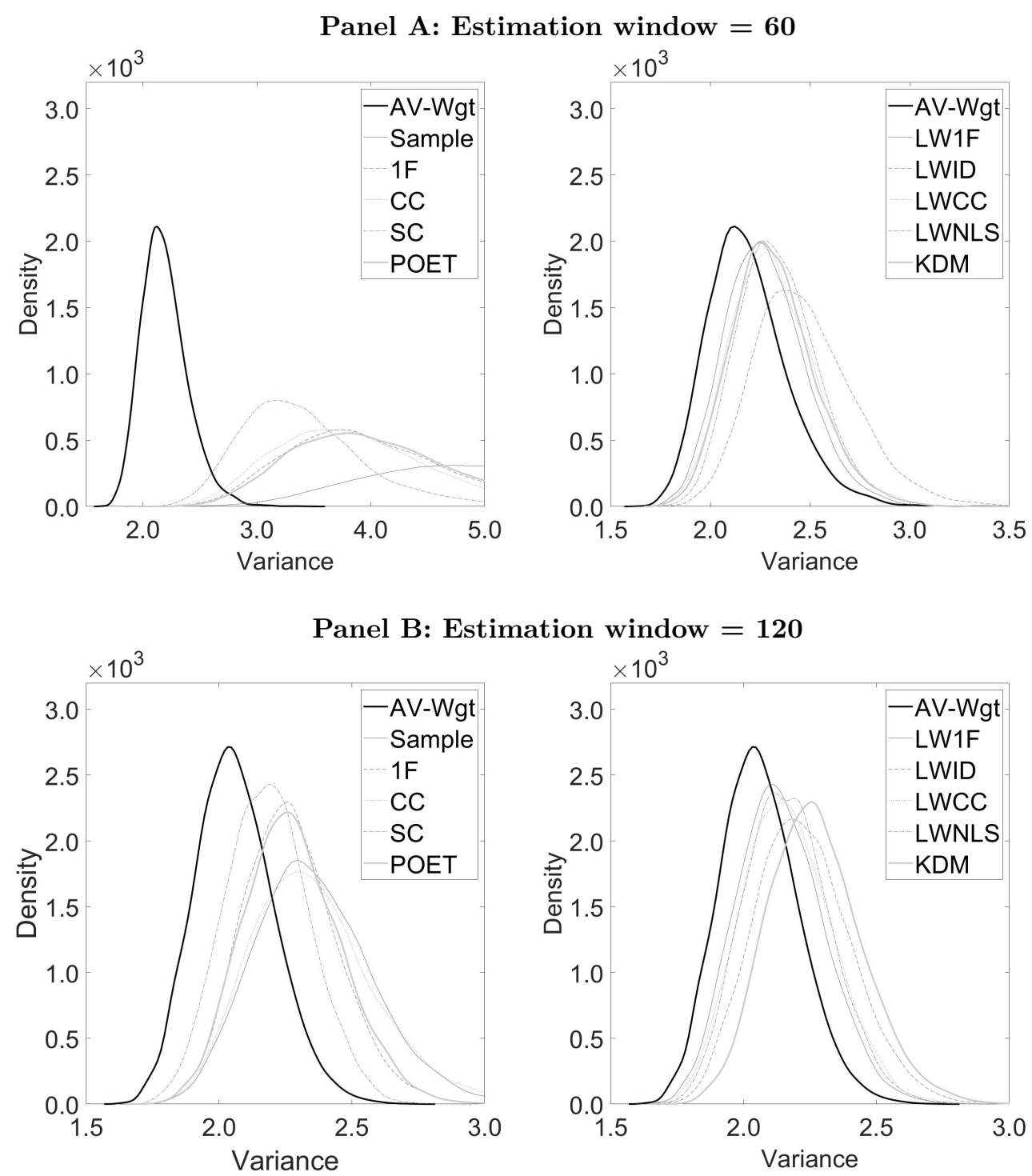

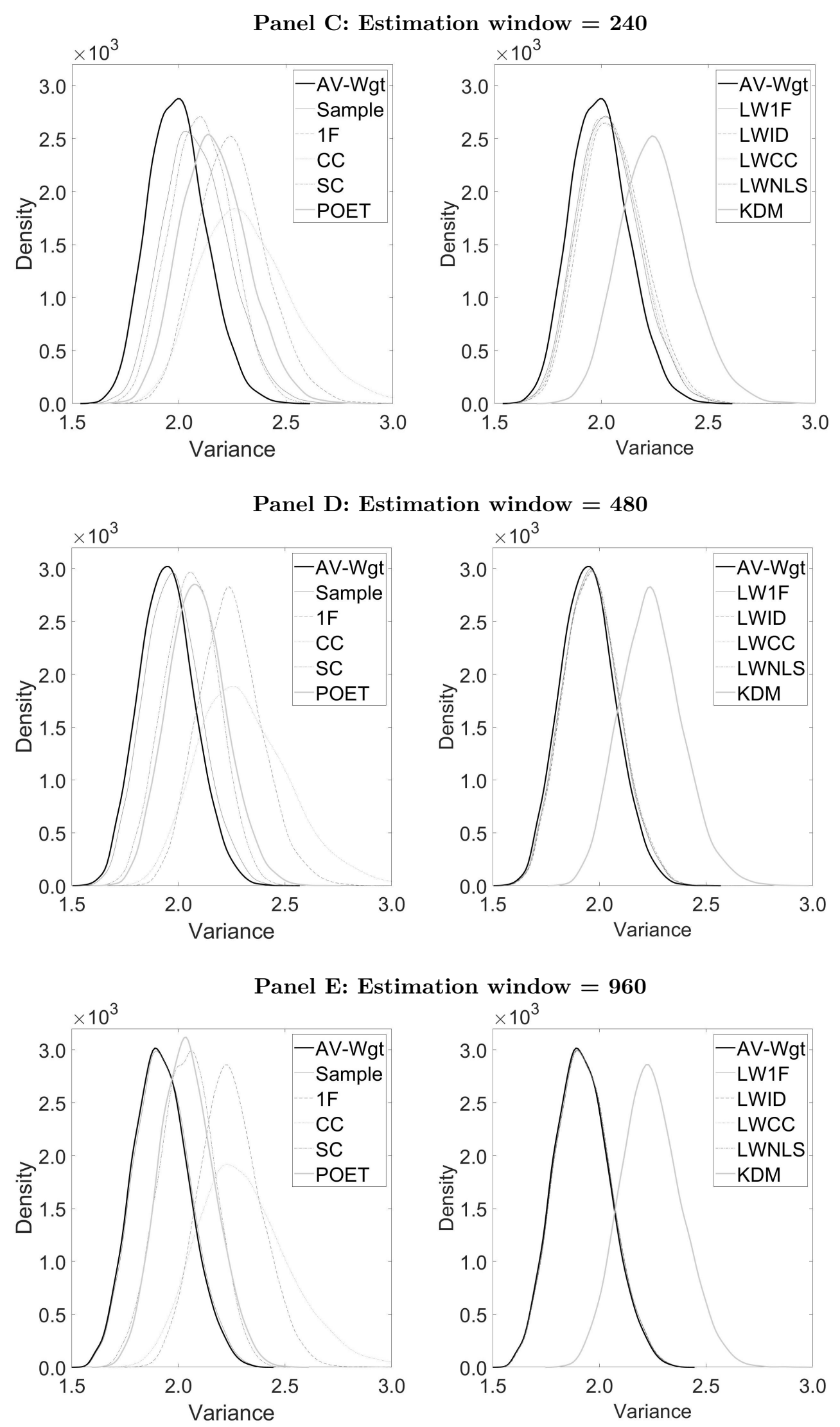
Figure A.5: Kernel densities of the out-of-sample variances of AV-Inv compared to established strategies and benchmarks

This figure plots the kernel densities of the out-of-sample variance of our averaging rule on the inverse covariance matrix (AV-Inv) level over 10,000 simulation runs in comparison to the single strategies (left column) and the benchmarks (right column) for the considered estimation windows of $\tau=\{60,120,240,480,960\}$. The out-of-sample variances are scaled by $10 \mathrm{e}+03$. The results for $\mathrm{AV}-\mathrm{Wgt}$ are based on averaging over the $M=6$ strategies from Panel B in Table 1 . For illustration purpose only, we exclude the ID strategy because it distorts the visualization of the remaining strategies.
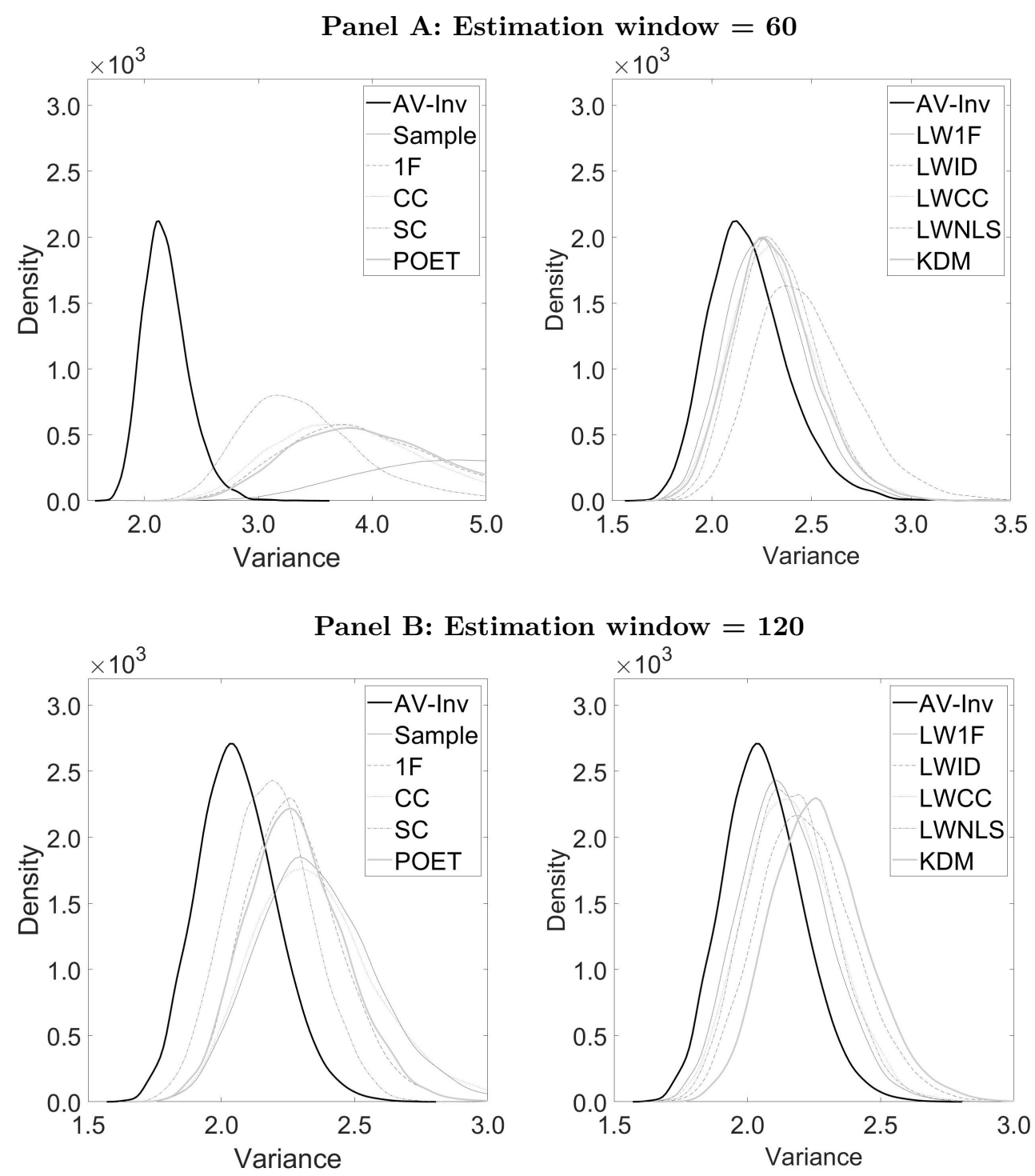

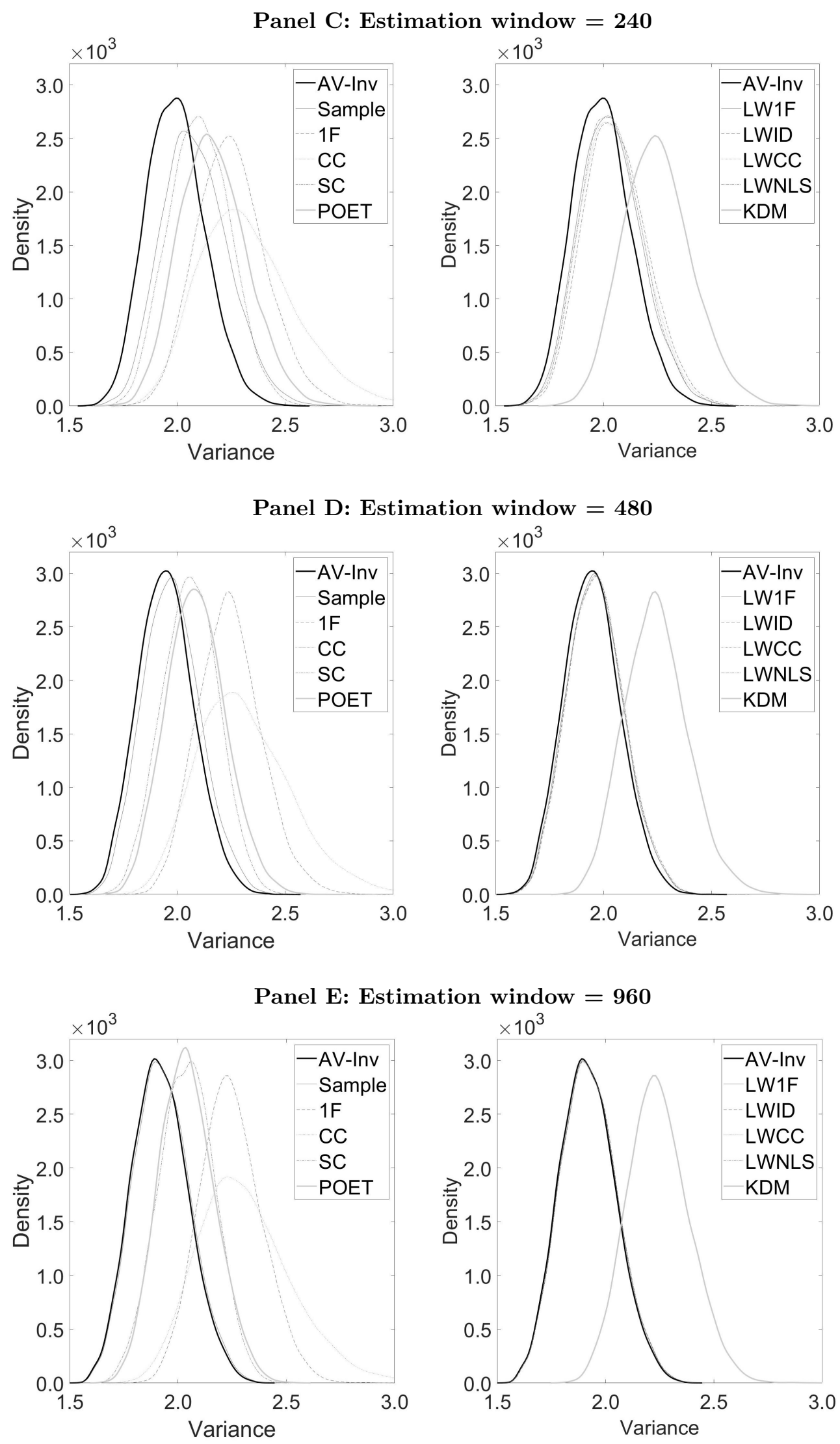
Figure A.6: Kernel densities of the out-of-sample variances of AV-Cov compared to established strategies and benchmarks

This figure plots the kernel densities of the out-of-sample variance of our averaging rule on the covariance matrix (AV-Cov) level over 10,000 simulation runs in comparison to the single strategies (left column) and the benchmarks (right column) for the considered estimation windows of $\tau=\{60,120,240,480,960\}$. The out-of-sample variances are scaled by $10 \mathrm{e}+03$. The results for AV-Wgt are based on averaging over the $M=6$ strategies from Panel B in Table 1. For illustration purpose only, we exclude the ID strategy because it distorts the visualization of the remaining strategies.

Panel C: Estimation window $=\mathbf{2 4 6 0}$

Figure A.7: Kernel densities of the out-of-sample Sharpe ratio of AV-Wgt compared to established strategies and benchmarks

This figure plots the kernel densities of the out-of-sample Sharpe ratio of our averaging rule on the portfolio weight (AV-Wgt) level over 10,000 simulation runs in comparison to the single strategies (left column) and the benchmarks (right column) for the considered estimation windows of $\tau=\{60,120,240,480,960\}$. The out-of-sample Sharpe ratios are scaled by $10 \mathrm{e}+03$. The results for AV-Wgt are based on averaging over the $M=6$ strategies from Panel B in Table 1. For illustration purpose only, we exclude the ID strategy because it distorts the visualization of the remaining strategies.
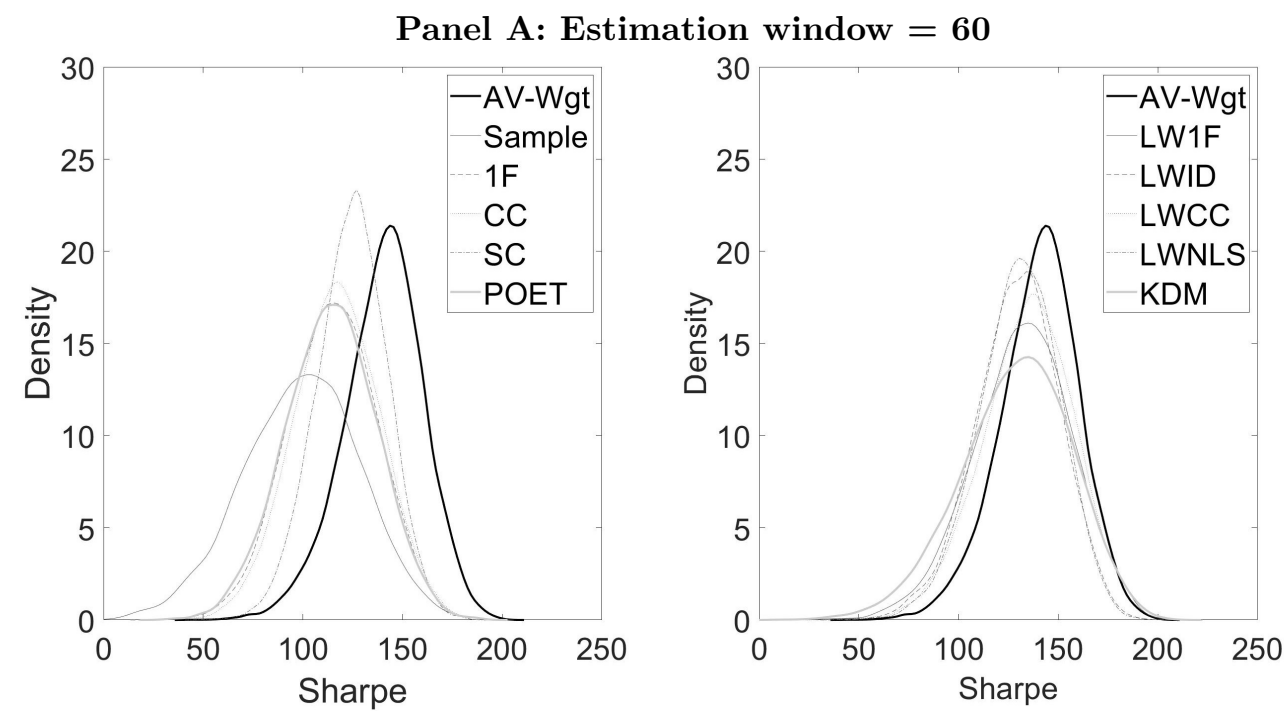

Panel B: Estimation window $=120$
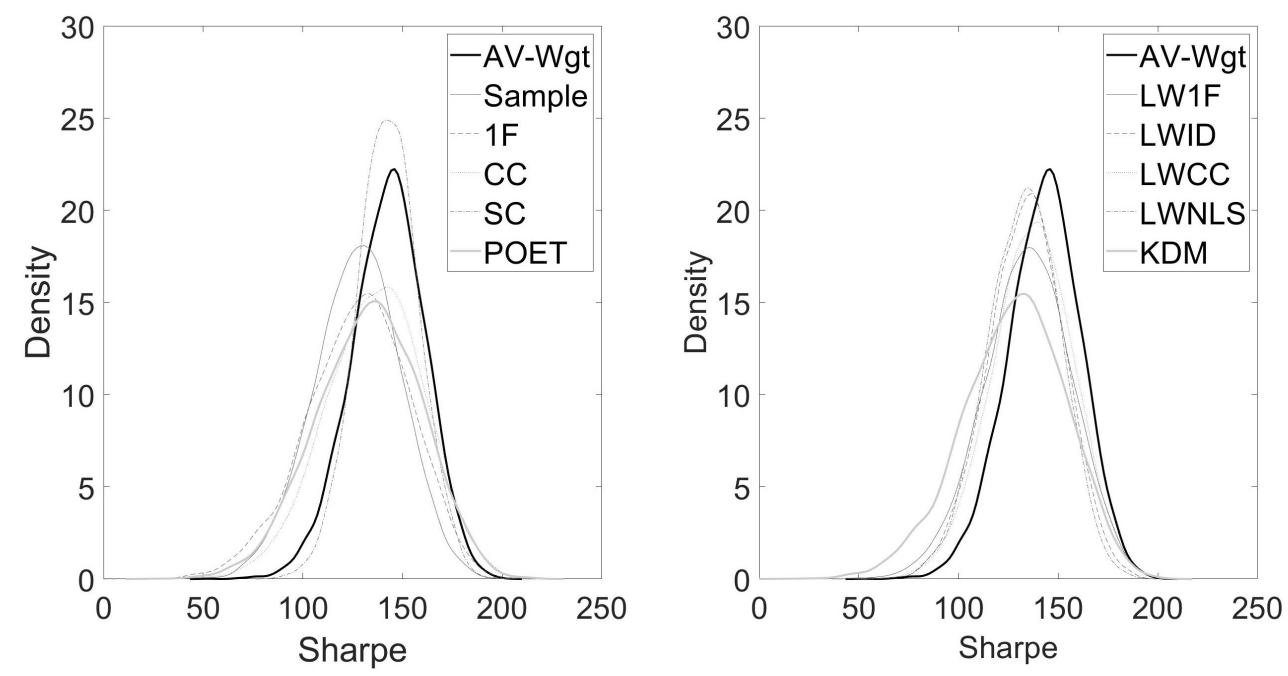
Panel C: Estimation window $=\mathbf{2 4 0}$
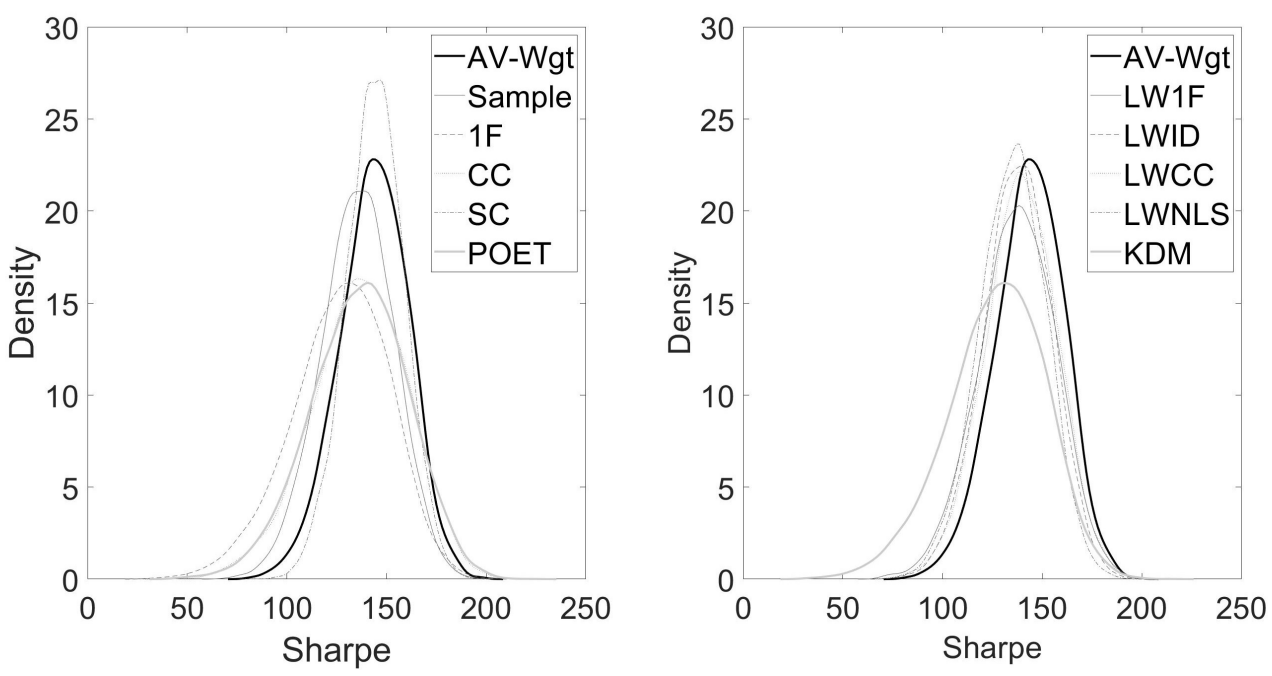

Panel D: Estimation window $=480$
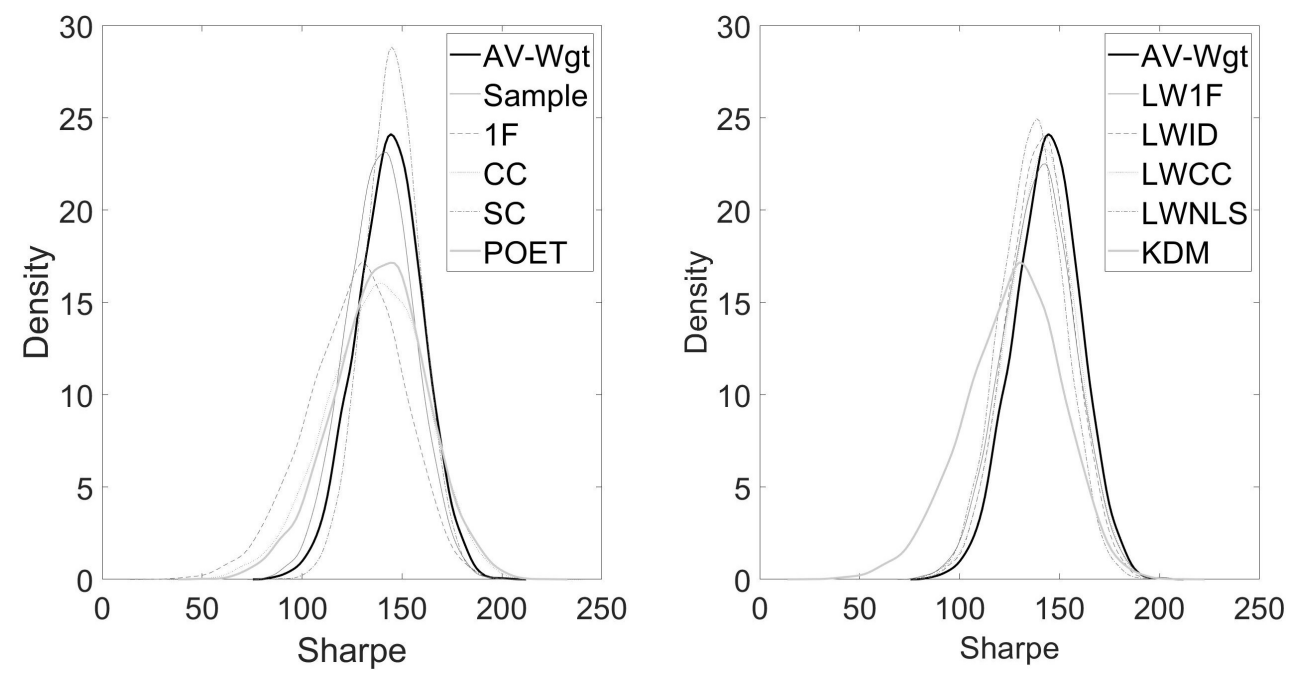

Panel E: Estimation window $=960$
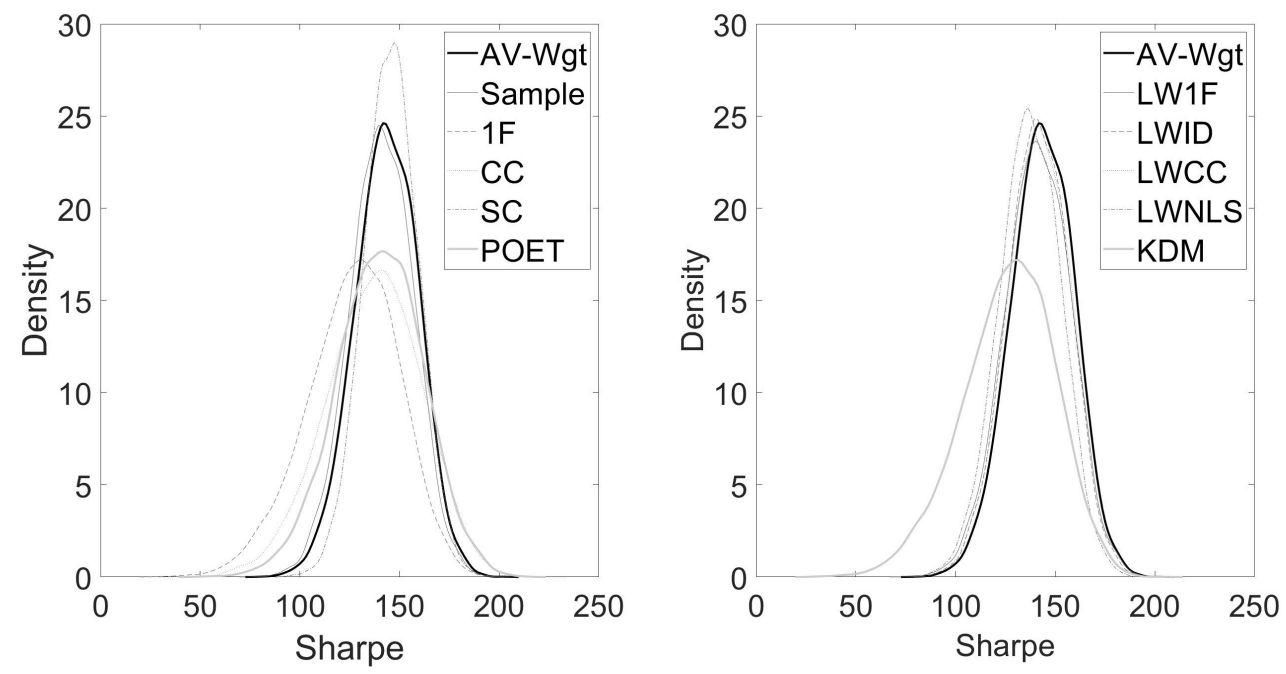
Figure A.8: Kernel densities of the out-of-sample Sharpe ratio of AV-Inv compared to established strategies and benchmarks

This figure plots the kernel densities of the out-of-sample Sharpe ratio of our averaging rule on the inverse covariance matrix (AV-Inv) level over 10,000 simulation runs in comparison to the single strategies (left column) and the benchmarks (right column) for the considered estimation windows of $\tau=\{60,120,240,480,960\}$. The out-of-sample Sharpe ratios are scaled by $10 \mathrm{e}+03$. The results for $\mathrm{AV}$-Inv are based on averaging over the $M=6$ strategies from Panel $\mathrm{B}$ in Table 1. For illustration purpose only, we exclude the ID strategy because it distorts the visualization of the remaining strategies.
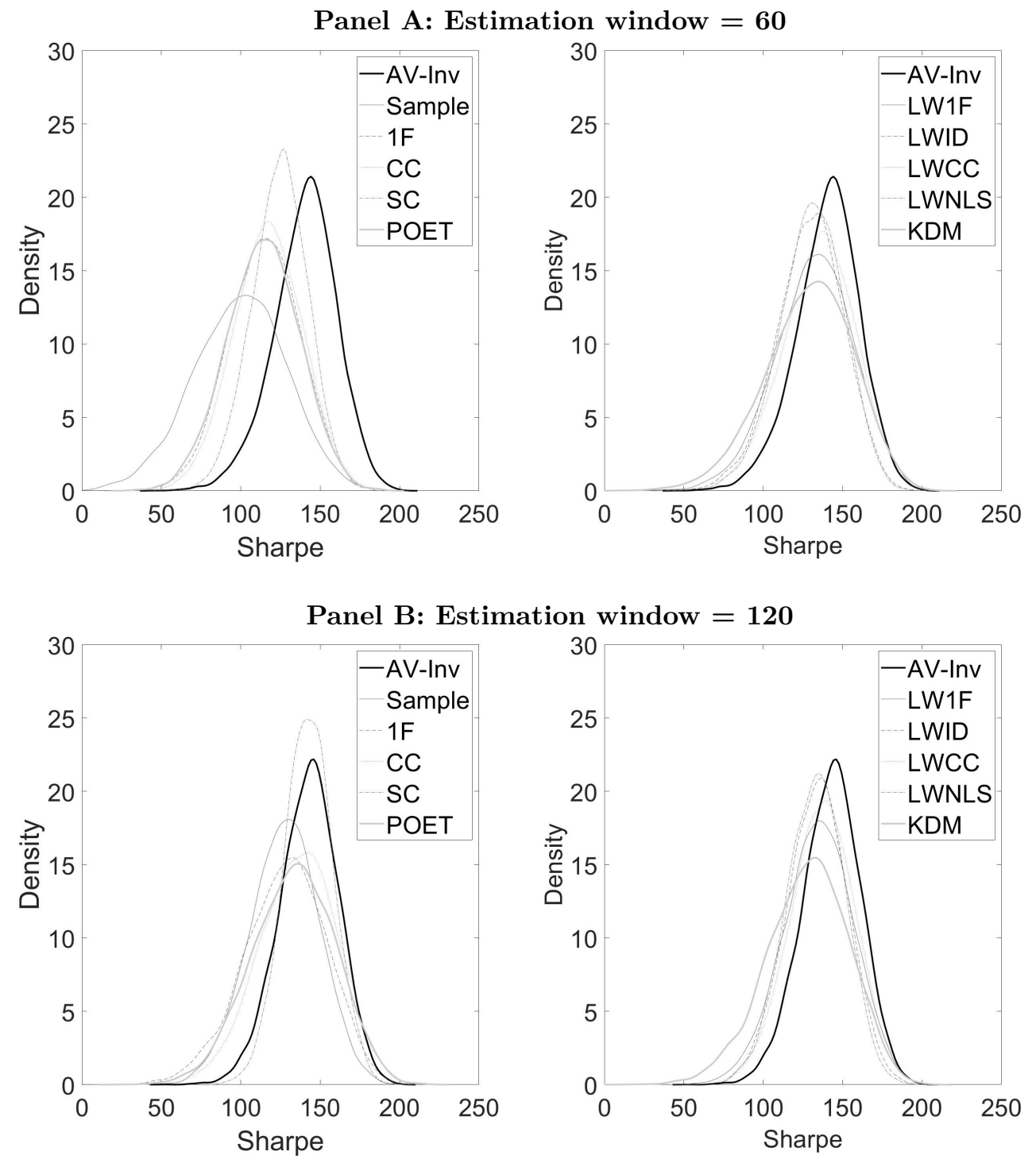
Panel C: Estimation window $=\mathbf{2 4 0}$
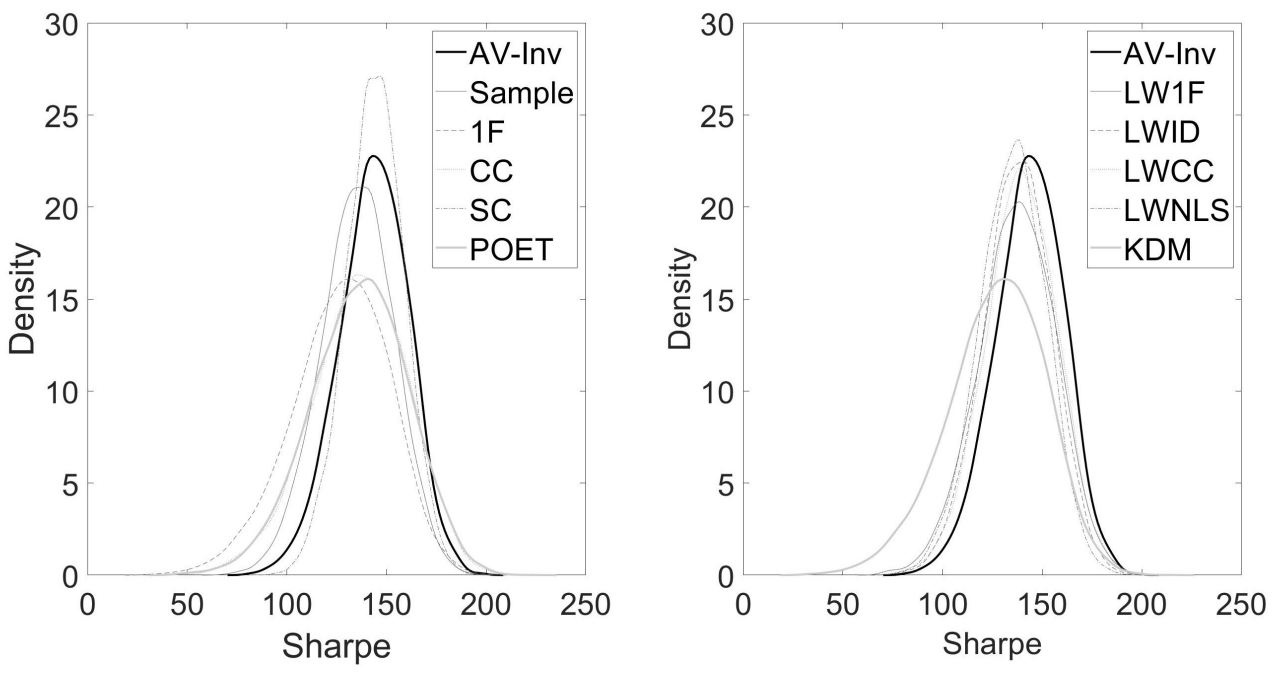

Panel D: Estimation window $=\mathbf{4 8 0}$
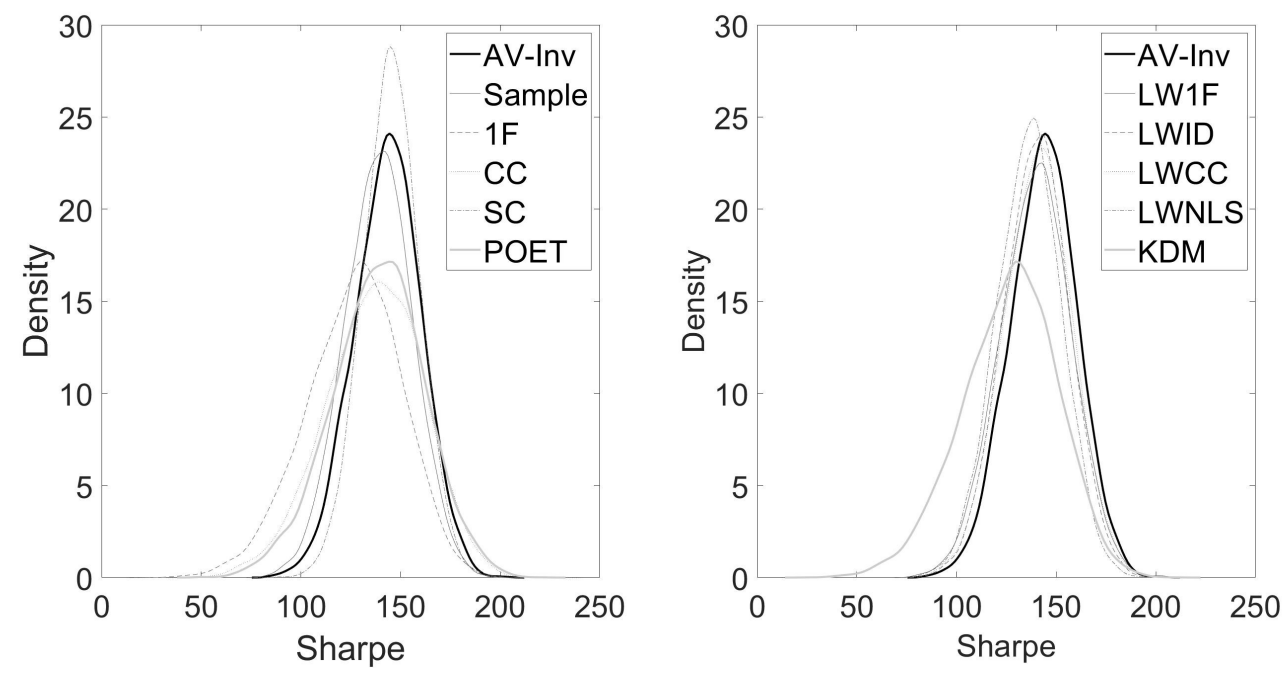

Panel E: Estimation window $=960$
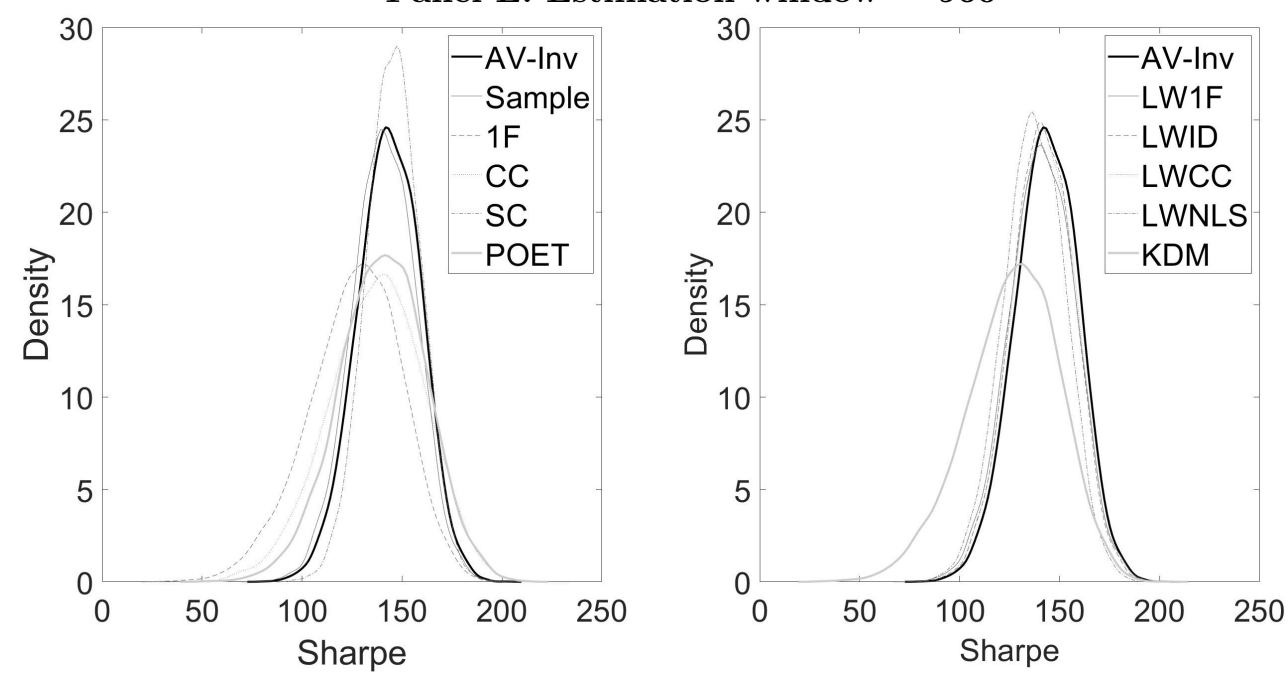
Figure A.9: Kernel densities of the out-of-sample Sharpe ratio of AV-Cov compared to established strategies and benchmarks

This figure plots the kernel densities of the out-of-sample Sharpe ratio of our averaging rule on the covariance matrix (AV-Cov) level over 10,000 simulation runs in comparison to the single strategies (left column) and the benchmarks (right column) for the considered estimation windows of $\tau=\{60,120,240,480,960\}$. The out-of-sample Sharpe ratios are scaled by $10 \mathrm{e}+03$. The results for AV-Cov are based on averaging over the $M=6$ strategies from Panel B in Table 1. For illustration purpose only, we exclude the ID strategy because it distorts the visualization of the remaining strategies.
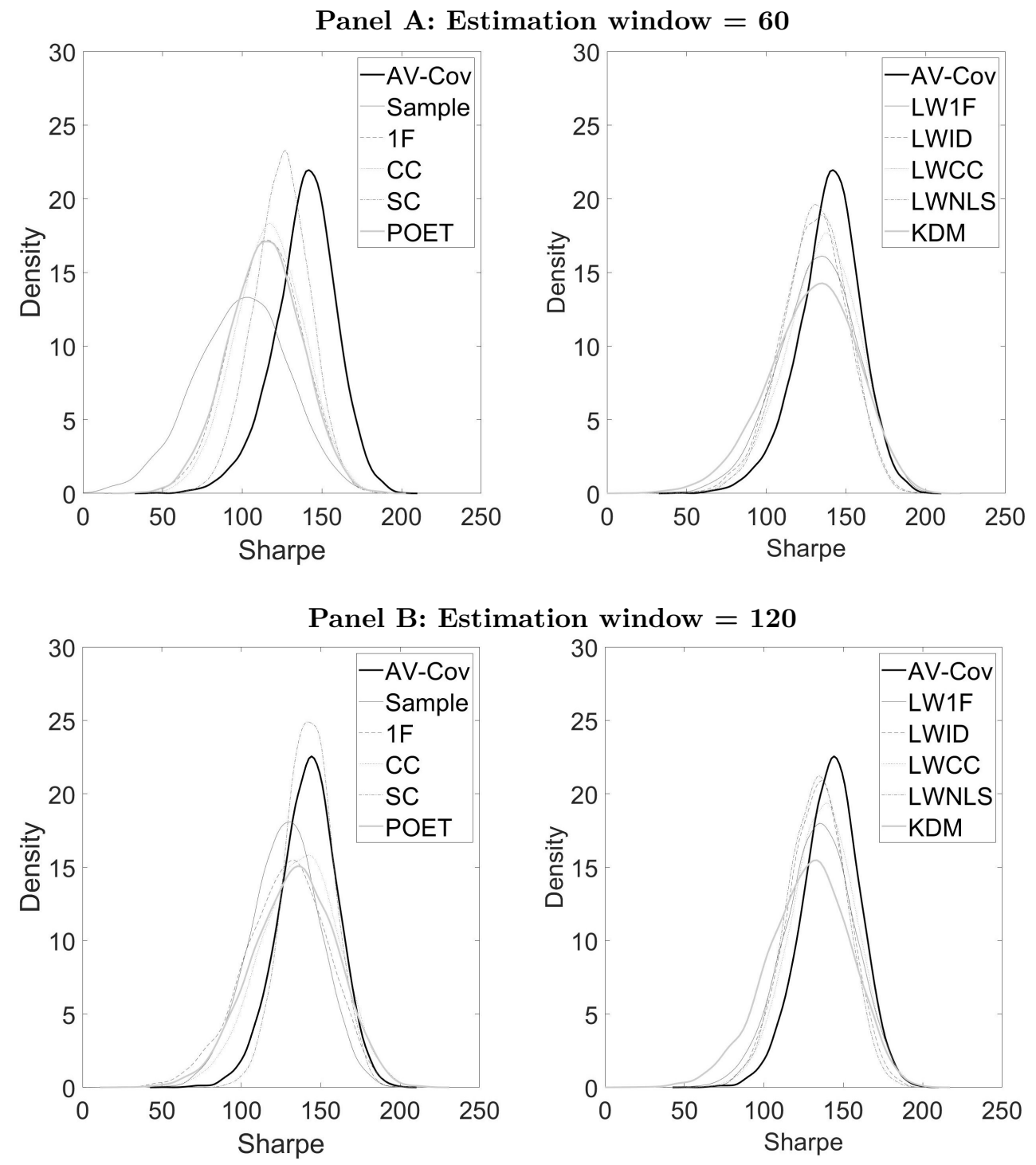
Panel C: Estimation window $=\mathbf{2 4 0}$


Panel D: Estimation window $=\mathbf{4 8 0}$
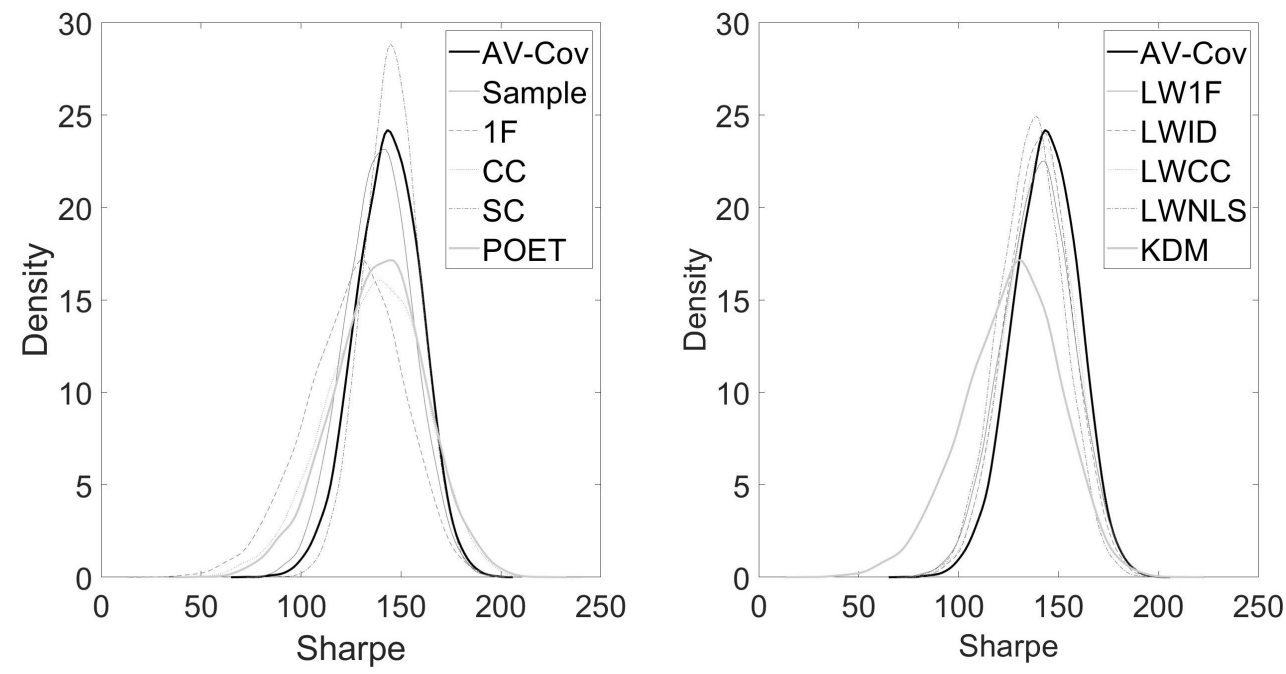

Panel E: Estimation window $=960$
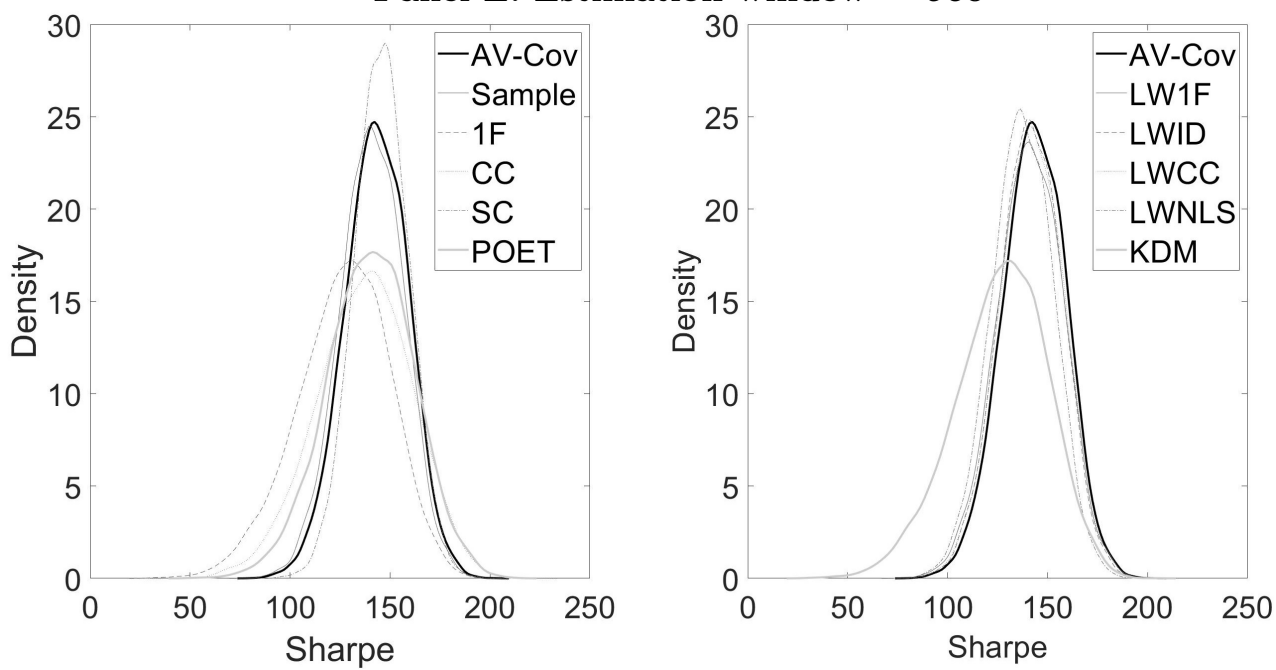


\section{A.3 Results on monthly data frequency}

Following Ledoit and Wolf (2017), we change the excess return frequency from daily to monthly. While keeping the out-of-sample period the same from 06/1976-06/2019, we use the longer available history to estimate the covariance matrix over $T=120$ most recent months, i.e. 10 years of data, as common in the literature. The data sets as described in Table A.2 are the same for the Fama-French factor-mimicking and industry portfolios. We reduce the CRSP data set to the 30 largest stocks, representing the Dow Jones Industrial Average index. We thus ensure that the number of assets $N$ remains smaller than the available monthly observations $\tau$. In July of each year we select the 30 largest stocks in terms of market capitalization from all NYSE, AMEX, and NASDAQ stocks in the Center for Research in Security Prices (CRSP) database that fulfill certain filter criteria. We filter out stocks with a price of less than $\$ 5$ as for daily observations, as well as stocks with missing excess returns in the 120 preceding months or the 12 months subsequent to the selection date. The stocks constitute the investment universe for one year. For our rolling-sample procedure, we set the estimation window to $\tau=120$ observations, corresponding to ten years of monthly data. Starting in June 1966, we estimate the portfolio weights of each strategy using only the information in the estimation window, which comprises the most recent $\tau$ excess returns. We hold the estimated portfolio weights constant for a one-month holding period, and save the corresponding out-of-sample excess returns. We then move the estimation window forward by a month and repeat the aforementioned procedure over the entire sample period. The specification is thus similar to the simulation study with an estimation window of $\tau=120$. The remaining details are as described in the main text.

We find that the results on a monthly data frequency are qualitatively similar to the ones presented in Section 4 for daily observations. Our averaging rule achieves on all averaging levels comparable variances and Sharpe ratios. Comparing AV-Wgt to the benchmarks in Panel C of Table A.3 we find that AV-Wgt achieves on all five data sets lower variances than LWNLS, being significantly lower for four out of five. The variance is also lower or similar to the other shrinkage estimators LW1F, LWID, LWCC, or KDM. In terms of out-of-sample risk-adjusted performance, AV-Wgt achieves consistently higher Sharpe ratios compared to the ID strategy in Panel B of Table A.4. In most cases, the performance is also superior, or at least similar, to the benchmarks in Panel C. The monthly average turnover for AV-Wgt as shown in Table A.5 is larger than for the benchmarks for the smaller $6 \mathrm{FF}$ or $10 \mathrm{Ind}$ portfolios but tend to be lower for the $25 \mathrm{FF}$ or $30 \mathrm{Ind}$ portfolios and 30 stocks (STOCK30). As with daily returns, the short interest of AV-Wgt in Table A.6 is the smallest of all strategies (excluding ID and SC) for the 30 industry portfolio and the stocks, and on a similar level as LW1F and LWNLS for the remaining portfolios. Summing up, our averaging rule on monthly data constitutes a further improvement over the existing strategies and shrinkage approaches similar as for daily data. 
Table A.2: List of the empirical data sets for monthly data

This table lists the data sets for the empirical evaluation of our proposed averaging rule in comparison to existing portfolio strategies, their abbreviations, the number of assets in each data set, and the data sources. All data sets span the period 06/1966 - 06/2019, constitute monthly data, and apply in the case of portfolio data the value weighting scheme to the respective constituents. Data from Kenneth French is taken from his website (http://mba.tuck.dartmouth. edu/pages/faculty/ken.french/data_library.html) and represents different cuts of the U.S. stock market. The STOCK30 data set contains the 30 largest single stocks in terms of market capitalization in July of every year after filtering out stocks that have a price of less than $\$ 5$, or missing returns in the preceding 120 and subsequent 12 trading months to the selection date. All stock prices are taken from the Center of Research in Security Prices (CRSP).

\begin{tabular}{llcccc}
\hline$\#$ & Data set & Abbreviation & N & Source \\
\hline 1 & $\begin{array}{l}6 \text { Fama and French portfolios of firms sorted by size and } \\
\text { book-to-market }\end{array}$ & $6 \mathrm{FF}$ & 6 & K. French \\
2 & $\begin{array}{l}25 \text { Fama and French portfolios of firms sorted by size } \\
\text { and book-to-market }\end{array}$ & $25 \mathrm{FF}$ & 25 & K. French \\
3 & 10 industry portfolios representing the U.S. stock market & $10 \mathrm{Ind}$ & 10 & K. French \\
4 & 30 industry portfolios representing the U.S. stock market & 30 3nd & 30 & K. French \\
5 & 30 Stocks with the largest market capitalization & STOCK30 & 30 & CRSP \\
\hline
\end{tabular}


Table A.3: Monthly out-of-sample variances in empirical data sets

This table reports the monthly out-of-sample variances of our averaging rule on the portfolio weight (AV-Wgt), the inverse (AV-Inv), and the covariance (AV-Cov) level in Panel A, of the single strategies in Panel B, and of the benchmarks in Panel C. The results are shown for the Fama-French factor-mimicking and industry portfolios, as well as the STOCK30 data set. The out-of-sample period is from 06/1976 - 06/2019. The abbreviations for the strategies in Panel B and $\mathrm{C}$, as well as for the data sets, are reported in Table1 1 and 7 , respectively. We report statistical significance for the null hypothesis wherein the (log) expected out-of-sample portfolio variance of the averaging rule on the portfolio weight level, AV-Wgt, is greater than that of the non-linear shrinkage approach of Ledoit and Wolf (2017), LWNLS: $i$ : $H_{0}: \log \left(\hat{\sigma}_{A V-W g t}^{2}\right)-\log \left(\hat{\sigma}_{L W N L S}^{2}\right) \leq 0$. We follow Ledoit and Wolf (2011) and use their proposed bootstrap procedure, using a block length of 5 and 1,000 iterations. Statistical significance at the 1, 5, and $10 \%$ level is denoted by $* * *, * *$, and $*$, respectively.

\begin{tabular}{|c|c|c|c|c|c|}
\hline & $6 \mathrm{FF}$ & $25 \mathrm{FF}$ & 10Ind & 30Ind & STOCK30 \\
\hline \multicolumn{6}{|c|}{ Panel A: Averaging rules } \\
\hline AV-Wgt & 13.9847 & 12.2703 & 11.0577 & 10.6377 & 12.8335 \\
\hline AV-Inv & 13.9706 & 12.2774 & 11.0736 & 10.6116 & 12.8364 \\
\hline $\mathrm{AV}-\mathrm{Cov}$ & 14.2756 & 12.2592 & 11.0897 & 10.6961 & 12.8539 \\
\hline \multicolumn{6}{|c|}{ Panel B: Single strategies } \\
\hline Sample & 14.3117 & 13.7620 & 11.4345 & 12.6086 & 15.2445 \\
\hline $1 \mathrm{~F}$ & 20.5461 & 29.6997 & 12.5939 & 13.0024 & 15.4703 \\
\hline $\mathrm{CC}$ & 19.3826 & 32.6633 & 13.5744 & 16.4668 & 15.9903 \\
\hline ID & 22.1005 & 23.7947 & 16.5779 & 20.7166 & 17.7152 \\
\hline $\mathrm{SC}$ & 17.5683 & 16.6497 & 11.3624 & 11.5421 & 12.7908 \\
\hline POET & 14.9073 & 13.2813 & 11.6811 & 11.7436 & 14.0681 \\
\hline \multicolumn{6}{|c|}{ Panel C: Benchmarks } \\
\hline LW1F & 14.4877 & 12.7579 & 11.2287 & 10.9466 & 13.3622 \\
\hline LWID & 14.3537 & 12.0929 & 10.9141 & 10.9056 & 13.3110 \\
\hline LWCC & 17.6658 & 19.2178 & 11.3035 & 11.3676 & 12.7354 \\
\hline LWNLS & $14.3093^{* *}$ & $12.7787^{* *}$ & 11.1915 & $11.1344^{* *}$ & $13.3429 * *$ \\
\hline $\mathrm{KDM}$ & 20.5461 & 29.6988 & 12.5939 & 13.0024 & 15.4703 \\
\hline
\end{tabular}


Table A.4: Monthly out-of-sample Sharpe ratios in empirical data sets

This table reports the monthly out-of-sample Sharpe ratios of our averaging rule on the portfolio weight (AV-Wgt), the inverse (AV-Inv), and the covariance (AV-Cov) level in Panel A, of the single strategies in Panel B, and of the benchmarks in Panel C. The results are shown for the Fama-French factor-mimicking and industry portfolios, as well as the STOCK30 data set. The out-of-sample period is from 06/1976 - 06/2019. The abbreviations for the strategies in Panel $\mathrm{B}$ and $\mathrm{C}$, as well as for the data sets, are reported in Table 1 and 7 , respectively. We use the 30-day T-bill rate as the risk-free rate. We report statistical significance for the null hypothesis wherein the Sharpe ratio of the averaging rule on the portfolio weight level, AV-Wgt, is greater than that of the $1 / \mathrm{N}$ portfolio, corresponding to the ID strategy: $H_{0}: S R_{A V-W g t}-S R_{I D} \leq 0$. We follow Ledoit and Wolf $(2008)$ and use their proposed bootstrap procedure, using a block length of 5 and 1,000 iterations. Statistical significance at the 1, 5, and $10 \%$ level is denoted by $* * *, * *$, and $*$, respectively.

\begin{tabular}{lccccr}
\hline & $6 \mathrm{FF}$ & $25 \mathrm{FF}$ & $10 \mathrm{Ind}$ & 30Ind & STOCK30 \\
\hline Panel A: & Averaging rules & & & & \\
AV-Wgt & 0.2668 & 0.2632 & 0.2035 & 0.1872 & 0.1667 \\
AV-Inv & 0.2681 & 0.2636 & 0.2030 & 0.1870 & 0.1672 \\
AV-Cov & 0.2664 & 0.2673 & 0.2005 & 0.1850 & 0.1634
\end{tabular}

Panel B: Single strategies

\begin{tabular}{|c|c|c|c|c|c|}
\hline Sample & 0.2799 & 0.2925 & 0.2023 & 0.1609 & 0.1103 \\
\hline $1 \mathrm{~F}$ & 0.1571 & 0.1608 & 0.2124 & 0.1863 & 0.1748 \\
\hline $\mathrm{CC}$ & 0.1608 & 0.1456 & 0.1936 & 0.1811 & 0.1634 \\
\hline ID & $0.1631^{* * *}$ & $0.1656^{* * *}$ & 0.1681 & 0.1535 & 0.1578 \\
\hline $\mathrm{SC}$ & 0.1775 & 0.1816 & 0.1964 & 0.1824 & 0.1739 \\
\hline POET & 0.3135 & 0.2711 & 0.1818 & 0.1733 & 0.1829 \\
\hline \multicolumn{6}{|c|}{ Panel C: Benchmarks } \\
\hline LW1F & 0.2678 & 0.2825 & 0.2051 & 0.1830 & 0.1402 \\
\hline LWID & 0.2308 & 0.2754 & 0.2090 & 0.1983 & 0.1434 \\
\hline LWCC & 0.1887 & 0.2152 & 0.2028 & 0.1912 & 0.1555 \\
\hline LWNLS & 0.2600 & 0.2522 & 0.2013 & 0.1912 & 0.1584 \\
\hline KDM & 0.1571 & 0.1608 & 0.2124 & 0.1863 & 0.1748 \\
\hline
\end{tabular}


Table A.5: Average monthly turnover in empirical data sets based on monthly observations

This table reports the average monthly turnover of our averaging rule on the portfolio weight (AVWgt), the inverse (AV-Inv), and the covariance (AV-Cov) level in Panel A, of the single strategies in Panel B, and of the benchmarks in Panel C. The results are shown for the Fama-French factor-mimicking and industry portfolios, as well as the STOCK30 data set. The out-of-sample period is from 06/1976 - 06/2019. The abbreviations for the portfolio strategies in Panels B and $\mathrm{C}$, as well as for the data sets, are reported in Table 1 and 7 , respectively. Turnover is measured as the average percentage of total wealth traded in each period. The reported numbers are in percentages.

\begin{tabular}{lcrrrr}
\hline & $6 \mathrm{FF}$ & $25 \mathrm{FF}$ & 10Ind & 30Ind & STOCK30 \\
\hline Panel A: & Averaging rules & & & & \\
AV-Wgt & 18.23 & 40.99 & 11.02 & 20.52 & 40.27 \\
AV-Inv & 18.47 & 41.57 & 10.99 & 20.28 & 40.75 \\
AV-Cov & 23.865 & 39.87 & 11.56 & 21.35 & 40.75 \\
\multicolumn{1}{l}{ Panel B: Single } & strategies & & & & \\
Sample & 21.88 & 79.46 & 15.47 & 45.92 & 29.74 \\
1F & 12.36 & 24.54 & 9.25 & 14.79 & 12.69 \\
CC & 10.32 & 23.23 & 7.54 & 13.96 & 10.64 \\
ID & 1.47 & 1.67 & 2.26 & 2.85 & 4.36 \\
SC & 4.09 & 6.96 & 4.87 & 6.74 & 8.98 \\
POET & 27.22 & 43.96 & 16.37 & 23.96 & 17.85 \\
\multicolumn{2}{l}{ Panel C: Benchmarks } & & & & \\
LW1F & 19.73 & 55.67 & 13.60 & 29.33 & 19.06 \\
LWID & 9.03 & 32.09 & 10.41 & 26.35 & 18.92 \\
LWCC & 10.89 & 28.60 & 9.63 & 22.20 & 14.26 \\
LWNLS & 19.56 & 54.99 & 13.78 & 28.95 & 19.14 \\
KDM & 12.36 & 24.54 & 9.25 & 14.79 & 12.69 \\
\hline
\end{tabular}


Table A.6: Average monthly short interest in empirical data sets

This table reports the average monthly short interest of our averaging rule on the portfolio weight (AV-Wgt), the inverse (AV-Inv), and the covariance (AV-Cov) level in Panel A, of the single strategies in Panel B, and of the benchmarks in Panel C. The results are shown for the Fama-French factor-mimicking and industry portfolios, as well as the STOCK30 data set. The out-of-sample period is from 06/1976 - 06/2019. The abbreviations for the strategies in Panel B and $\mathrm{C}$, as well as for the data sets, are reported in Table 1 and 7 , respectively. Short interest is measured by the average amount of wealth that is held in short positions. The reported numbers are in percentages.

\begin{tabular}{|c|c|c|c|c|c|}
\hline & $6 \mathrm{FF}$ & $25 \mathrm{FF}$ & 10Ind & 30Ind & STOCK30 \\
\hline \multicolumn{6}{|c|}{ Panel A: Averaging rules } \\
\hline AV-Wgt & 108.23 & 195.87 & 36.47 & 57.28 & 27.98 \\
\hline AV-Inv & 111.16 & 198.87 & 35.54 & 56.94 & 27.93 \\
\hline AV-Cov & 107.66 & 199.12 & 35.76 & 60.05 & 29.03 \\
\hline \multicolumn{6}{|c|}{ Panel B: Single strategies } \\
\hline Sample & 140.33 & 328.78 & 64.29 & 159.97 & 85.36 \\
\hline $1 \mathrm{~F}$ & 88.05 & 158.19 & 53.68 & 82.89 & 44.79 \\
\hline $\mathrm{CC}$ & 76.54 & 144.93 & 41.03 & 77.17 & 38.58 \\
\hline ID & 0.00 & 0.00 & 0.00 & 0.00 & 0.00 \\
\hline $\mathrm{SC}$ & 0.00 & 0.00 & 0.00 & 0.00 & 0.00 \\
\hline POET & 193.73 & 269.34 & 70.25 & 96.15 & 53.75 \\
\hline \multicolumn{6}{|c|}{ Panel C: Benchmarks } \\
\hline LW1F & 123.08 & 260.15 & 59.75 & 113.88 & 55.48 \\
\hline LWID & 63.08 & 171.05 & 41.86 & 97.42 & 51.26 \\
\hline LWCC & 75.19 & 159.06 & 38.56 & 86.43 & 40.78 \\
\hline LWNLS & 116.09 & 253.59 & 56.81 & 105.21 & 51.73 \\
\hline KDM & 88.05 & 158.18 & 53.68 & 82.89 & 44.79 \\
\hline
\end{tabular}

